\title{
Factorizable Representation of Current Algebra -Non commutative extension of the Lévy-Kinchin formula and cohomology of a solvable group with values in a Hilbert Space-
}

\author{
By
}

Huzihiro ARAKI

\begin{abstract}
A notion of factorizable representation is defined and all factorizable representations of a commutative group of functions as well as those of the current commutation relations and canonical commutation relations are explicitly given in the continuous tensor product space (i.e. the Fock space).

The formula for a state functional of a factorizable representation is a non-commutative extension of the Levy-Kinchin formula in probability theory.

In the course of analysis, the most general form of a first order cocycle of any solvable group with values in a Hilbert space is determined. Non trivial cohomologies appear by two entirely different mechanism, namely a topological one on infinite dimensional space and an algebraic one on a finite dimensional space.

The immaginary part of an inner product of such cocycle is a second order cocycle. The condition that it is a coboundary is discussed.
\end{abstract}

\section{$\S 1 . \quad$ Introduction}

A factorizable representation has been discussed for commutative groups of functions and canonical commutation relations [1], [2]. Here we extend this notion to the current algebra, which is of some interest in elementary particle physics. Given a Lie group $G$, we consider, so to speak, a continuous direct product of copies of $G$, each associated with a point on a measure space $X$. In the commutative case, $G$ is the additive group of reals. In the canonical commutation relations, we take $G$ as the group corresponding to the Lie algebra of one pair of canonical variables $p, q$ and an identity operator (times $i$ ). If a unitary representation of the big group is 
associated with a state, which splits up into product of states of the small group at every points, then the representation can be realized in the continuous tensor product space defined in [3]. We shall attempt a determination of the structure of such a representation.

It is shown that a representation is determined by specifying at each point $x$ of $X$, a unitary representation $Q_{x}(g)$ of $G$, a first order cocycle $\phi_{x}(g)$ with values in the representation space of $Q_{x}$, and a real valued function $c_{x}(g)$ whose coboundary should coincide with $\operatorname{Im}\left(\phi\left(g_{1}\right), \phi\left(g_{2}^{-1}\right)\right)$. The representing operator can be expressed in terms of Bose creation and annihilation operators as an exponential of $a^{*}(x) a(x), a^{*}(x)$ and $a(x)$ terms.

All cocycles $\phi$ for given unitary representation are obtained for any solvable group $G$. It is in general a sum of coboundary of a vector in a space $\bar{D}^{+}$which is somewhat larger than the representation space, and certain vectors in the adjoint representation space of $G$ (on its Lie algebra). The problem for a general $G$ can be reduced to the case of a semisimple $G$. The cocycles for a semisimple $G$ are not analyzed.

The problem of finding $c$ for a given $Q$ and $\phi$ is partially solved.

The general theory is applied to special examples, including the abelian case, the canonical commutation relations and current algebra over the rotation group.

\section{§2. Current Algebra}

Let $X$ be a measure space and $G$ be a connected Lie group with a finite dimensional Lie algebra $g$.

Definition 2.1. The current group $\mathrm{C}(G, X)$ of $G$ over $X$ is the group of all bounded G-valued measurable functions equipped with the pointwise group operations. The bounded function means that its range is contained in a compact subset of $G$. The measurability refers to the $\sigma$-fields $B_{X}$ of measurable sets in $X$ and the $\sigma$-field $B_{G}$ generated by compact sets of $G$.

Definition 2.2. The current algebra $\mathrm{C}(\mathrm{g}, X)$ of $\mathrm{g}$ over $X$ is the Lie algebra of all bounded measurable g-valued functions equipped with the pointwise Lie algebra operations. 
For each element $l$ of $\mathrm{C}(\mathrm{g}, X)$ the exponential $e^{l}$ of $\boldsymbol{l}$ is the element of $\mathrm{C}(G, X)$ defined pointwise by $\left[e^{\top}\right](x)=e^{l(x)}$, where the last exponential is the conventional exponential mapping from Lie algebra to Lie group.

Definition 2.3. A unitary representation $\mathrm{g} \in \mathrm{C}(G, X) \rightarrow \mathrm{U}(\mathrm{g})$ (a unitary operator on a Hilbert space $\mathfrak{S})$ is called continuous if $\mathrm{U}\left(e^{t l}\right)$ is strongly continuous in the real parameter $t$ for each fixed $l$ in $\mathrm{C}(\mathrm{g}, X)$.

Lemma 2.4. Let $\chi\left(X_{0}\right)$ be a characteristic function of a fixed measurable subset $X_{0}$ of $X$ and let $g^{x\left(X_{0}\right)}$ be the function belonging to $\mathrm{C}(G, X)$ which is equal to $g$ on $X_{0}$ and to 1 on $X-X_{0}$. Then $g \in G$ $\rightarrow \mathrm{U}\left(g^{x\left(X_{0}\right)}\right)$ is a representation of the group $G$, which is continuous in the ordinary sense if $\mathrm{U}$ is continuous.

Proof. The group representation property follows from $\left(g_{1} g_{2}\right)^{{ }^{x}\left(X_{0}\right)}$ $=g_{1}{ }^{x\left(\sim_{0}\right)} g_{2}{ }^{x\left(y_{0}\right)}$. As for the continuity, we use the fact that (a) because of the representation property $\mathrm{U}(\mathrm{g})=\mathrm{U}\left(\mathrm{gg}_{0}^{-1}\right) \mathrm{U}\left(\mathrm{g}_{0}\right)$, it suffices to prove the continuity at $g=1$, and (b) if $\mathfrak{l}_{1} \cdots \mathfrak{l}_{n}$ is a basis of $\mathfrak{g},\left(t_{1} \cdots t_{n}\right)$ $\rightarrow e^{t_{1} \mathrm{I}_{1} \ldots} e^{t_{n} \mathrm{I}_{n}}$ gives a homeomorphism of a neighbourhood of 0 in $R^{n}$ and a neighbourhood of 1 in $G$. By assumption each $\mathrm{U}\left(e^{t_{j} l_{j}}\right)$ is continuous in $t$ and uniformly bounded, where $\boldsymbol{l}_{j}(x)$ is equal to $\mathfrak{l}_{j}$ on $X_{0}$ and to 1 on $X \cdots X_{0}$. By using the estimate of the following general form

$$
\begin{aligned}
& \left\|A\left(t_{1}\right) A\left(t_{2}\right) \Phi-A\left(t_{1}{ }^{0}\right) A\left(t_{2}{ }^{0}\right) \Phi\right\| \\
& \quad \leqq\left\|A\left(t_{1}\right)\right\|\left\|A\left(t_{2}\right) \Phi-A\left(t_{2}{ }^{\circ}\right) \Phi\right\|+\left\|\left[A\left(t_{1}\right)-A\left(t_{1}{ }^{\circ}\right)\right] A\left(t_{2}{ }^{\circ}\right) \Phi\right\|,
\end{aligned}
$$

we have the strong continuity of $\mathrm{U}\left(e^{t_{1} I_{1}}\right) \cdots \mathrm{U}\left(e^{t} n^{l_{n}}\right)$ in $\left(t_{1} \cdots t_{n}\right)$.

Definition 2.5. A functional $\mathrm{E}$ over $\mathrm{C}(G, X)$ is a mapping from $\mathrm{C}(G, X)$ to complex numbers. A triplet of a Hilbert space $\mathfrak{S}, a$ unitary representation $\mathrm{U}$ of $\mathrm{C}(G, X)$ on $\mathfrak{S}$ and a cyclic vector $\Psi$ in $\mathfrak{S}$ (namely a vector $\Psi$ such that vectors $\mathrm{U}(\mathrm{g}) \Psi, g \in \mathrm{C}(G, X)$ span $\mathfrak{E}$ ) is said to be canonically associated with $\mathrm{E}$ if

$$
\mathrm{E}(\mathrm{g})=(\Psi, \mathrm{U}(\mathrm{g}) \Psi), \quad \mathrm{g} \in \mathrm{C}(G, X)
$$

Theorem 2.6. A triplet $(\mathfrak{E}, \mathrm{U}, \Psi)$ canonically associated with a functional $\mathrm{E}$ exists if and only if $\mathrm{E}$ satisfies the following positivity 
condition: $\mathrm{E}\left(\mathrm{g}_{i}{ }^{-1} \mathrm{~g}_{j}\right), i=1, \cdots, n, j=1, \cdots, n$, is a nonnegative matrix for any choice of $\mathrm{g}_{i}, i=1, \cdots, n$ from $\mathrm{C}(G, X)$. For given $\mathrm{E}$, the triplet is unique up to a unitary equivalence. The unitary representation $\mathrm{U}$ is continuous if and only if $\mathrm{E}\left(\mathrm{g}_{1} e^{t} \mathrm{~g}_{2}\right)$ is continuous in the real parameter $t$ for every fixed $\mathrm{g}_{1}, \mathrm{~g}_{2} \in \mathrm{C}(G, X)$ and $\boldsymbol{l} \in \mathrm{C}(\mathrm{g}, X)$.

The necessity of the conditions for $\mathrm{E}$ is obvious. The existence and uniqueness of the triplet is easily proved by a standard method.

Since $\mathrm{U}\left(e^{t}\right)$, being unitary, is uniformly bounded, the continuity of its matrix elements between a total set $\mathrm{U}(\mathrm{g}) \Psi$ implies the weak continuity of $\mathrm{U}\left(e^{t}\right)$ itself and hence its strong continuity.

Definition 2.7. A functional $\mathrm{E}$ is called an expectation functional if $\mathrm{E}(1)=1$, the positivity condition is satisfied and $\mathrm{E}\left(\mathrm{g}_{1} e^{t} \mathrm{~g}_{2}\right)$ is continuous in $t$.

An expectation functional satisfies

$$
\begin{aligned}
\mathrm{E}(\mathrm{g})^{*} & =\mathrm{E}\left(\mathrm{g}^{-1}\right) \\
|\mathrm{E}(\mathrm{g})| & \leqq 1
\end{aligned}
$$

which immediately follows from the positivity condition with $n=2$, $\mathrm{g}_{1}=1$ and $\mathrm{g}_{2}=\mathrm{g}$.

The definitions introduced in this section are related to customary notations in the following way.

If $G$ is taken to be the additive group of real numbers, then $C(G, X)$ is a vector space of functions. The functional $\mathrm{E}$ for such $C(G, X)$ has been extensively treated in the literature.

If $G$ is the Heisenberg group, namely if $g$ is spanned by three elements $\hat{\mathfrak{q}}, \hat{\mathfrak{p}}, \mathfrak{i}$ satisfying $[\hat{\mathfrak{p}}, \hat{\mathfrak{q}}]=\mathfrak{i},[\hat{\mathfrak{q}}, \mathfrak{i}]=[\hat{\mathfrak{p}}, \hat{\mathfrak{i}}]=0$ and if we require $\mathrm{U}\left(e^{\mathrm{i} f}\right)$, for any real valued bounded measurable function $f$, to be an identity operator times a number $\exp i \int_{X} f(x) \mathrm{d} x$, where $\mathrm{d} x$ is a measure on $X$, then we are dealing with a representation of canonical commutation relations. The last condition may be expressed in terms of the functional $\mathrm{E}$ by

$$
\mathrm{E}\left(\mathrm{g} e^{\mathrm{i} f}\right)=\mathrm{E}(\mathrm{g}) \exp i \int f(x) \mathrm{d} x .
$$

In this case, the customary notation is

$$
\mathrm{U}(e \hat{q} f)=\mathrm{U}(f)=e^{i \phi(f)}=e^{i \int \phi(x) f(x) \mathrm{d} x}
$$




$$
\mathrm{U}(\hat{e} \hat{p} f)=V(f)=e^{i \pi(f)}=e^{i \int \pi(x) f(x) \mathrm{d} x} .
$$

If $\mathfrak{g}$ is a Lie algebra of antihermitian matrices $\left(\mathfrak{g}_{i j}\right)$, then a standard notation is

$$
\mathrm{U}\left(e^{g}\right)=\exp \sum_{k l} \psi^{+}(x)_{k} \psi(x)_{l} g(x)_{k l} \mathrm{~d} x .
$$

For $\gamma \in \mathfrak{g}$, the expression $\psi^{+}(x) \gamma \psi(x)$ is the operator valued distribution defined through a generator of one parameter subgroup:

$$
\int\left(\psi^{+}(x) \gamma \psi(x)\right) f(x) \mathrm{d} x=\left.\frac{\mathrm{d}}{\mathrm{d} t} \mathrm{U}\left(e^{t \gamma f}\right)\right|_{t=0} .
$$

If $\gamma$ is antihermitian, $i$ times this operator is selfadjoint. The current commutation relation is

$$
\left[\psi^{+}(x) \gamma_{1} \psi(x), \psi^{+}(y) \gamma_{2} \psi(y)\right]=\delta(x-y) \psi^{+}(x)\left[\gamma^{1}, \gamma^{2}\right] \psi(x)
$$

which is to be understood as

$$
\begin{gathered}
{\left[\int \psi^{+}(x) \gamma_{1} \psi(x) f_{1}(x) \mathrm{d} x, \int \psi^{+}(y) \gamma_{2} \psi(y) f_{2}(y) \mathrm{d} y\right]} \\
=\int \psi^{+}(x)\left[\gamma_{1}, \gamma_{2}\right] \psi(x) f_{1}(x) f_{2}(x) \mathrm{d} x
\end{gathered}
$$

and holds on a dense set of vectors depending on $\gamma_{1} f_{1}$ and $\gamma_{2} f_{2}$.

The use of $\psi^{+}$and $\psi$ comes from the fact that (2.9) is formally implied by

$$
\begin{gathered}
{\left[\psi^{+}(x), \psi(y)\right]_{ \pm}=\delta(x-y)} \\
{\left[\psi^{+}(x), \psi^{+}(y)\right]_{ \pm}=[\psi(x), \psi(y)]_{ \pm}=0}
\end{gathered}
$$

where either + or - commutation relations are assumed, $[A, B]_{+}$ $=A B+B A,[A, B]_{-}=A B-B A$, and they lead to the same equation (2.9).

Sometimes, one is interested in a locally compact space $X$ and the group of $G$-valued measurable bounded functions on $X$ with a bounded support. The following analysis can be modified to accomodate such cases.

\section{§3. Factorizable Functional and Type I Factorization}

Definition 3.1. An expectation functional $\mathrm{E}$ of $\mathrm{C}(G, X)$ is finitely factorizable if 


$$
\mathrm{E}\left(\mathrm{g}_{1} \mathrm{~g}_{2}\right)=\mathrm{E}\left(\mathrm{g}_{1}\right) \mathrm{E}\left(\mathrm{g}_{2}\right)
$$

whenever the support of $\mathrm{g}_{1}$ is disjoint from the support of $\mathrm{g}_{2}$. Here the support of a function $\mathrm{g}(x)$ is the set of $x \in X$ for which $\mathrm{g}(x) \neq 1$. If $\left\{X_{i} ; i=1, \cdots, n\right\}$ is a partition of the set $X$ into a finite number of subsets in $B_{X}$, then the subgroup of functions $\mathrm{g}(x)$ with supports in $X_{i}$ may be identified with $\mathrm{C}\left(G, X_{i}\right) . \mathrm{C}(G, X)$ is then a direct product of (mutually commuting groups) $\mathrm{C}\left(G, X_{i}\right), i=1, \cdots, n$.

If we denote the restriction of the functional $\mathrm{E}$ to $\mathrm{C}\left(G, X_{i}\right)$ by $\mathrm{E}_{i}$, it automatically satisfies a condition for an expectation functional of $\mathrm{C}\left(G, X_{i}\right)$. For $Y \in B_{X}$ and $\mathrm{g} \in \mathrm{C}(G, X)$, let $\mathrm{g}^{\mathrm{x}(Y)}$ denote the element of $\mathrm{C}(G, X)$ which is equal to $\mathrm{g}$ on $Y$ and to 1 on $X-Y$. For a finitely factorizable functional $\mathrm{E}$ and for a finite partition $\left\{X_{i}\right\}$ of $X$, we have

$$
\mathrm{E}(\mathrm{g})=\prod_{i=1}^{n} \mathrm{E}_{i}\left(\mathrm{~g}^{\mathrm{x}\left(X_{i}\right)}\right) .
$$

Theorem 3.2. Let $(\mathfrak{E}, \mathrm{U}, \Psi)$ and $\left(\mathfrak{S}_{i}, \mathrm{U}_{i}, \Psi_{i}\right)$ be triplets canonically associated with expectation functionals $\mathrm{E}$ and $\mathrm{E}_{i}$, respectively. Let

$$
\begin{gathered}
\hat{\mathfrak{K}}=\bigotimes_{i=1}^{n} \mathfrak{S}_{i}, \quad \hat{\Psi}=\bigotimes_{i=1}^{n} \Psi_{i} \\
\hat{\mathrm{U}}\left(\prod_{i=1}^{n} \mathrm{~g}_{i}\right)=\stackrel{\bigotimes}{i=1}_{i=1}^{n} \mathrm{U}_{i}\left(\mathrm{~g}_{i}\right), \quad \mathrm{g}_{i} \in \mathrm{C}\left(G, X_{i}\right)
\end{gathered}
$$

where $\otimes$ denotes the tensor product. If $\mathrm{E}$ is finitely factorizable, then $(\hat{\mathfrak{C}}, \hat{\mathrm{U}}, \hat{\Psi})$ is unitarily equivalent to $(\mathfrak{S}, \mathrm{U}, \Psi)$.

This follows from (3.2) and Theorem 2.6.

For any subset $X_{1}$ of $X$ in $B_{X}$, we consider partition of $X$ into $X_{1}$ and its complement $X_{1}^{\mathrm{c}} \equiv X_{2}$. By the unitary equivalence of $\mathfrak{E}=\mathfrak{L}_{1} \otimes \mathfrak{S}_{2}$ with $\mathfrak{S}, \mathscr{B}\left(\mathfrak{S}_{1}\right) \otimes 1$ defines a type I factor on $\mathfrak{E}$, which we shall write $\mathrm{R}\left(X_{1}\right)$. Here $\mathscr{B}\left(\mathfrak{E}_{1}\right)$ denotes the set of all bounded operators of $\mathfrak{S}_{1}$.

Theorem 3.3. Let $\mathrm{E}$ be finitely factorizable. For any finite partition $\left\{X_{i}, i=1, \cdots, n\right\}, X_{i} \in B_{X}$ of a given $X_{0} \in B_{X},\left\{\mathrm{R}\left(X_{i}\right), i=1\right.$, $\cdots, n\}$ is a type I factorization of $\mathrm{R}\left(X_{0}\right)$ for which $\Psi$ is a product vector. The operator $\mathrm{U}(\mathrm{g})$ belongs to $\mathrm{R}\left(X_{0}\right)$ if the support of $\mathrm{g}$ is in $X_{0}$ and is factorizable operator with respect to this factorization: $\mathrm{U}(\mathrm{g})=\Pi \mathrm{U}\left(\mathrm{g}^{\mathrm{x}\left(X_{i}\right)}\right), \mathrm{U}\left(\mathrm{g}^{\mathrm{x}\left(X_{i}\right)}\right) \in \mathrm{R}\left(X_{i}\right)$. 
This follows from Theorem 3.2 if we consider the partition $\left\{X_{0}^{\mathrm{c}}, X_{1}, \cdots, X_{n}\right\}$ as a common subpartition of $\left\{X_{0}, X_{0}^{\mathrm{c}}\right\}$ and $\left\{X_{i}, X_{i}^{\mathrm{c}}\right\}$, $i=1, \cdots, n$.

Definition 3.4. An expectation functional $\mathrm{E}$ of $\mathrm{C}(G, X)$ is said to be $\sigma$-factorizable if the following condition is satisfied:

If $\left\{X_{i}\right\}$ is a countable partition of $X$ into measurable sets, then

$$
\mathrm{E}(\mathrm{g})=\prod_{i} \mathrm{E}\left(\mathrm{g}_{i}\right)
$$

where the product converges irrespective of the ordering (the absolute convergence).

Definition 3.5. A subset $X_{0}$ of $X$ is called E-null set if $X_{0} \in B_{X}$ and $\mathrm{E}(\mathrm{g})=1$ for an arbitrary $\mathrm{g} \in \mathrm{C}\left(G, X_{0}\right)$. The set of all $\mathrm{E}$-null sets is denoted by $N_{X}^{\mathrm{E}}$.

Lemma 3.6. Let $\mathrm{E}$ be a $\sigma$-factorizable functional. If $X_{1} \in N_{X}^{\mathrm{E}}$, $X_{2} \in B_{X}$ and $X_{2} \subset X_{1}$, then $X_{2} \in N_{X}^{\mathrm{E}}$. If $X_{i} \in N_{X}^{\mathrm{E}}$, then $\cup X_{i} \in N_{X}^{\mathrm{E}}$ where the index set is countable.

Proof. The first statement follows from $\mathrm{C}\left(G, X_{2}\right) \subset \mathrm{C}\left(G, X_{1}\right)$. For the second statement, let $X_{0}=\bigcup_{i} X_{i}$ and $Y_{i}=X_{i}-\left(\bigcup_{j<i} Y_{j}\right)$. Since $Y_{i} \subset X_{i}, Y_{i} \in N_{X}^{\mathrm{E}}$. Since $X_{0}$ is the union of disjoint $Y_{i}$, Definition 3.4 implies $\mathrm{E}(\mathrm{g})=\prod_{i} \mathrm{E}\left(\mathrm{g}_{i}\right)=1$ for all $\mathrm{g} \in \mathrm{C}\left(G, X_{0}\right)$. Hence $X_{0} \in N_{X}^{\mathrm{E}}$.

Definition 3.7. The carrier Boolean algebra $B_{X}^{\mathrm{E}}$ of a factorizable $\mathrm{E}$ is the Boolean algebra obtained from the $\sigma$ field $B_{X}$ by identifying any two sets which differ by an addition and a subtruction of E-null sets.

Definition 3.8. $\mathrm{E}$ is called separable if, for any measurable partition $\left\{X_{\alpha}\right\}$ of $X, X_{\alpha}$ belongs to $N_{X}^{\mathrm{E}}$ except for a countable number of $\alpha$.

Lemma 3.9. If $\mathrm{E}$ is $\sigma$-factorizable and separable, then $B_{X}^{\mathrm{E}}$ is a complete Boolean algebra satisfying a countable chain condition.

Proof. Since $B_{X}$ is a $\sigma$-field (i.e. Boolean $\sigma$-algebra), $B_{X}^{\mathrm{E}}$ is also a Boolean $\sigma$-algebra because of Lemma 3.6. Next, if $X_{\mu} \in B_{X}$ is given for every ordinals $\mu$ between $\mu_{0}$ and 1 such that $X_{\mu} \supset X_{\nu}$ and $X_{\mu}-X_{\nu} \notin N_{X}^{\mathrm{E}}$ for $\mu>\nu\left(A-B \equiv A \cap B^{\mathrm{c}}\right)$, then $\mu_{0}$ must be countable. 
This follows from separability because $Y_{\mu} \equiv X_{\mu \cdot 1}-X_{\mu} \notin N_{X}^{\mathrm{E}}$ is mutually disjoint. Thus $B_{X}^{\mathrm{E}}$ satisfies the countable chain condition. Finally, let $X_{\mu} \in B_{X}$ be given. We show the existence of their least upper bound in $B_{X}^{\mathrm{E}}$. We define an ordinal $\mu_{0}$, the pair of the set $Y_{\mu}$ and an index $\alpha(\mu)$ for every ordinal $\mu<\mu_{0}$ by the transfinite induction in such a way that $Y_{\mu} \in B_{X}, Y_{\mu} \supset Y_{\nu}$ if $\mu>\nu, Y_{\mu}-Y_{\nu} \notin N_{X}^{\mathrm{E}}$ if $\mu>\nu$, $Y_{\mu}=\bigcup_{\nu<\mu} X_{\alpha(\nu)}$, and $X_{\alpha}-\bigcup_{\mu<\mu_{0}} Y_{\mu} \in N_{X}^{\mathrm{E}}$ for any $\alpha$.

If this is achieved, $\mu_{0}$ must be countable and $Y_{\mu_{0}} \equiv \bigcup_{\mu<\mu_{0}} Y_{\mu}$ is the lowest upper bound of $\left\{X_{\alpha}\right\}$ in $B_{X}^{\mathrm{E}}$. Let $Y_{\nu}$ be defined for $\nu<\mu$. (If $\mu=1$, this assumption holds trivially.) Then $\mu$ must be countable. Hence $\underset{\nu<\mu}{\cup} Y_{\nu} \equiv Z_{\mu} \in B_{X}$. We look for $X_{\alpha}$ such that $X_{\alpha}-Z_{\mu} \notin N_{X}^{\mathrm{E}}$. If no such $\alpha$ exists, we set $\mu=\mu_{0}$. Otherwise choose one such index $\alpha(\mu)$ and define $Y_{\mu}=X_{\alpha(\mu)} \cup Z_{\mu}$. Then $Y_{\mu} \in B_{X}, Y_{\mu} \supset Y_{\nu}$ for $\mu>\nu$. Further, if $\mu>\nu, Y_{\mu}-Y_{\nu} \supset Y_{\mu}-Z_{\mu}=X_{\alpha(\mu)}-Z_{\mu} \notin N_{X}^{\mathrm{E}}$ and hence $Y_{\mu}-Y_{\nu}$ $\notin N_{X}^{\mathrm{E}}$. Then strictly increasing transfinite sequence $\{\alpha(\nu) ; \nu<\mu\}$, $\mu=1,2, \cdots$ must terminate by the axiom of well ordering and we have the desired result.

Definition 3.10. The discrete spectrum $S_{X}^{\mathrm{dE}}$ of $\mathrm{E}$ in $X$ is the set of points $x \in X$ such that $\mathrm{E}(\mathrm{g}) \neq 1$ for some $\mathrm{g} \in \mathrm{C}(G,\{x\})$.

$S_{X}^{\mathrm{dE}}$ is countable for a separable $\mathrm{E}$.

Definition 3.11. Let $\hat{N}_{X}^{\mathrm{E}}$ be the family of subsets of $X$ obtained as a union of an E-null set and a subset (including the empty set) of $S_{X}^{\mathrm{dE}}$. The carrier Boolean algebra $B_{X}^{\mathrm{cE}}$ of the continuous part of $\mathrm{E}$ is the $\sigma$-field obtained from the $\sigma$-field $B_{X}$ by identifying any two sets which differ by an addition and a subtraction of sets in $\hat{N}_{X}^{\mathrm{E}}$. (Another equivalent way is to consider the lattice of $Y-S_{X}^{\mathrm{dE}}, Y \in B_{X}$ modulo null sets.)

Lemma 3.12. $B_{X}^{\mathrm{cE}}$ is a complete, continuous Boolean algebra with countable chain condition. $B_{X}^{\mathrm{E}}$ is a direct product of $B_{X}^{\mathrm{cE}}$ and the countable atomic complete Boolean algebra $B_{X}^{\mathrm{d} E}$ generated by points of $S_{X}^{\mathrm{dE}}$.

Definition 3.13. A representation $\pi$ of a complete Boolean algebra $B$ by factors is a mapping from $Y \in B$ to a factor $\pi(Y)$ such that $\pi\left(Y^{\mathrm{c}}\right)=\pi(Y)^{\prime}$ and $\pi\left(\cup Y_{i}\right)=\left(\cup \pi\left(Y_{i}\right)\right)^{\prime \prime}$. 
Lemma 3.14. Let $\mathrm{E}$ be a $\sigma$-factorizable scparable functional, $(\mathfrak{S}, \mathrm{U}, \Psi)$ be as in Theorem 3.2 and $\mathrm{R}(Y)$ be defined as stated after Theorem 3.2. Then (1) $\mathrm{R}\left(X_{1}\right)=\mathrm{R}\left(X_{2}\right)$ if $X_{1} \equiv X_{2}$ in $B_{X}^{\mathrm{E}}$. For $Y \in B_{X}^{\mathrm{E}}$, define $\mathrm{R}(Y)=\mathrm{R}\left(X_{1}\right)$ where $X_{1}$ is any set in the equivalence class $Y$. (2) $Y \in B_{X}^{\mathrm{E}} \rightarrow \mathrm{R}(Y)$ is a representation of a complete Boolean algebra by type I factors.

Proof. If $Y \in N_{X}^{\mathrm{E}}$, then the corresponding $\mathfrak{S}_{Y}$ is one dimensional and (1) follows from Theorem 3.2. As long as the finite Boolean operations are concerned, $Y \in B_{X}^{\mathrm{E}} \rightarrow \mathrm{R}(Y)$ is a representation of the Boolean algebra $B_{X}^{\mathrm{E}}$ by type I factors, due to Theorem 3.2. Hence the only point to be proved is whether $\left(\cup_{\alpha} \mathrm{R}\left(X_{\alpha}\right)\right)^{\prime \prime}=\mathrm{R}\left(\cup_{\alpha} X_{\alpha}\right)$ for arbitrary $X_{\alpha}$. Because of the countable chain condition, it is enough to see this for countable number of mutually disjoint $X_{\alpha}$.

Let $X_{0}=\left(\bigcup_{a} X_{a}\right)^{c}$ and adjoin 0 into the index set. Consider $\left(\mathfrak{F}_{\mu}\right.$, $\left.\mathrm{U}_{\mu}, \Psi_{\mu}\right)$ corresponding to the restriction of $\mathrm{E}$ to $\mathrm{C}\left(G, X_{\mu}\right)$ and construct the incomplete infinite direct product $\mathfrak{S}_{2} \otimes \mathfrak{S}_{\mu}$ containing $\Psi \equiv$ $\otimes \Psi_{\mu}$. Because of Definition (3.4) (b), $\Pi\left(\Psi_{\mu}, \mathrm{U}_{\mu}\left(\mathrm{g}_{\mu}\right) \Psi_{\mu}\right)$ is absolutely convergent, and hence $\otimes \mathrm{U}_{\mu}\left(\mathrm{g}_{\mu}\right) \Psi_{\mu} \in \hat{\mathcal{S}}$. Hence $\otimes \mathrm{U}_{\mu}\left(\mathrm{g}_{\mu}\right) \equiv \hat{\mathrm{U}}(\mathrm{g})$ also exists as a unitary operator on $\hat{\mathfrak{S}}$. Moreover $(\hat{\Psi}, \hat{\mathrm{U}}(\mathrm{g}) \hat{\Psi})=\mathrm{E}(\mathrm{g})$. Hence $(\hat{\mathfrak{Z}}, \hat{\mathrm{U}}, \hat{\Psi})$ is unitarily equivalent to $(\mathfrak{E}, \mathrm{U}, \Psi)$ of Theorem 3.2 . Furthermore, clearly $\hat{\mathrm{R}}_{\mu}=\mathscr{B}\left(\mathfrak{S}_{\mu}\right) \otimes\left(\otimes_{\nu \mu} 1_{\nu}\right)$ and $\hat{\mathfrak{S}}=\mathfrak{S}_{\mu} \otimes\left(\otimes_{\nu \neq \mu} \mathfrak{S}_{\nu}\right)$ correspond to $\mathrm{R}\left(X_{\mu}\right)$ and the related decomposition of the Hilbert space. Hence $\mathrm{R}\left(\bigcup_{\alpha} X_{\alpha}\right)=\mathrm{R}\left(X_{0}\right)^{\prime}=\left(\bigcup_{\alpha} \mathrm{R}\left(X_{\alpha}\right)\right)^{\prime \prime}$.

Definition 3.15. The set of $Y \in B_{X}^{\mathrm{cE}}$ such that $\mathrm{R}(Y)$ is one dimensional is denoted by $B_{X}^{\mathrm{c} 1 \mathrm{E}}$. The corresponding support $S_{X}^{\mathrm{c} 1 \mathrm{E}}$ is the maximal element of $B_{X}^{\mathrm{clE}}$ (defined as a subset of $X$ modulo $\mathrm{E}-$-null sets).

Lemma 3.16. $B_{X}^{\mathrm{c} 1 \mathrm{E}}$ is a complete Boolean sublattice of $B_{X}^{\mathrm{cE}}$. If $Y \in B_{X}^{\mathrm{c} 1 \mathrm{E}}, Y_{1} \subset Y, Y_{1} \in B_{X}^{\mathrm{cE}}$, then $Y_{1} \in B_{X}^{\mathrm{c} 1 \mathrm{E}}$.

Proof. The first part follows from Lemma 3.14 and the second part from Theorem 3.2.

Definition 3.17. The carrier Boolean algebra of the continuous tensor product part of $\mathrm{E}$ is the $\sigma$ field obtained from $B_{X}^{\mathrm{E}}$ by identifying any two sets which differ by a finite number of additions and 
subtractions of E-null sets, sets in $B_{X}^{\mathrm{dE}}$ and sets in $B_{X}^{\mathrm{cl} E}$. (Another way is to consider the lattice of $Y-S_{X}^{\mathrm{dE}}-S_{X}^{\mathrm{cl}}, Y \in B_{X}$ modulo $\mathrm{E}-n u l l$ sets.) It is denoted by $B_{X}^{\mathrm{cTE}}$.

Lemma 3.18. Let $\mathrm{E}$ be a $\sigma$-factorizable separable functional, ( $(\mathfrak{E}$, $\mathrm{U}, \Psi)$ be as in Theorem 3.2 and $\mathrm{R}(Y)$ be defined as stated after Theorem 3.2. (1) $B_{X}^{\mathrm{E}}$ is a direct product of $B_{X}^{\mathrm{dE}}, B_{X}^{\mathrm{clE}}$ and $B_{X}^{\mathrm{cTE}}$. (2) $\mathrm{g} \in \mathrm{C}(G$, $\left.S_{X}^{\mathrm{cl}}\right) \rightarrow \mathrm{E}(\mathrm{g})$ is a character of the group $\mathrm{C}\left(G, S_{X}^{\mathrm{cl}}\right)$ and the corresponding Hilbert space is one dimensional, (3) $Y \in B_{X}^{\mathrm{cTE}} \rightarrow \mathrm{R}(Y)$ is a faithful representation of the complete Boolean algebra $B_{X}^{\mathrm{cTE}}$ by type I factors.

Proof. (1) follows from Definition 3.11, Lemma 3.12, Definition 3.15, Lemma 3.16 and Definition 3.17. (2) follows from Definition 3.15. (3) follows from Lemma 3.14 and Definition 3.15.

\section{§4. Realization of the Unitary Representation in the Fock Space}

We now analyze the structure of the unitary representation $\mathrm{U}(\mathrm{g})$ and obtain a concrete realization in terms of creation and annihilation operators in the Fock space. First we separate out the discrete part. If $x \in S_{X}^{\mathrm{dE}}$, then $\mathrm{U}(\mathrm{g}), \mathrm{g} \in \mathrm{C}(G,\{x\})$ is a cyclic continuous unitary representation of the group $G$ and we have

$$
\begin{gathered}
\mathfrak{S}=\mathfrak{E}_{\mathrm{c}} \otimes\left\{\otimes_{x} \mathfrak{S}_{x}\right\} \\
\mathrm{U}(\mathrm{g})=\mathrm{U}_{\mathrm{c}}(\mathrm{g}) \otimes\left\{\underset{x}{\left\{\mathrm{U}_{x}\right.}(\mathrm{g}(x))\right\}
\end{gathered}
$$

where $x$ in $\underset{x}{\otimes}$ runs over all points in $S_{X}^{\mathrm{dE}}$. Thus the discrete part is reduced to the study of continuous unitary representation of the Lie group $G$, which we do not have to study any further. We shall now turn our attention to the continuous part $\mathfrak{W}_{c}$ and $U_{c}$. To simplify the notation we omit the $\mathrm{c}$, in other words, the discrete part is assumed to be absent in the following discussion.

Next we can separate out the character part. Any $\mathrm{g} \in \mathrm{C}(G, X)$ is decomposed as $\mathrm{g}=\mathrm{g}_{1} \mathrm{~g}_{2}$, supp $\mathrm{g}_{1} \subset S_{X}^{1 \mathrm{E}}$, supp $\mathrm{g}_{2} \subset X-S_{X}^{1 \mathrm{E}}$. Then $\mathrm{U}(\mathrm{g})=\mathrm{U}\left(\mathrm{g}_{1}\right) \mathrm{U}\left(\mathrm{g}_{2}\right)$ where $\mathrm{U}\left(\mathrm{g}_{1}\right)$ is a character of the group $\mathrm{C}\left(G, S_{X}^{1 \mathrm{E}}\right)$. A typical example of a character of $\mathrm{C}\left(G, S_{X}^{1 E}\right)$ is given by

$$
\exp \int \log \chi_{x}(g(x)) \mathrm{d} \mu(x)
$$


where $\chi_{x}$ is a character of $G$ depending measurably on $x$ and $\mu$ is a measure.

As long as the Hilbert space structure is concerned, $U\left(g_{1}\right)$ is a multiple of identity. In the present paper, we concentrate our attention to the continuous tensor product part and hereafter omit the superfix cT (which is equivalent to assuming that the $S_{X}^{\mathrm{clE}}$ part as well as the discrete part are absent).

For continuous tensor product part, the analysis of the continuous complete Boolean algebra of type I factors [3] reveals the following structure. $\mathfrak{S}$ is an exponential of a Hilbert space $\mathfrak{L}$. There exists a faithful representation of the complete Boolean algebra $B_{X}^{\mathrm{E}}$ by projections on $\mathscr{L}: Y \in B_{X} \rightarrow \mathrm{P}(Y), \mathrm{P}(Y)=0$ if and only if $\mathrm{R}(Y)=1$, that is $Y \in N_{X}^{\mathrm{E}}$. The most general forms of a product vector and a bounded product operator on $\mathfrak{S}$ are known.

We start with the analysis of the projection valued measure $\mathrm{P}(Y)$ on $\&$.

Lemma 4.1. There exists a countable partition of $X$ into mutually disjoint $X_{n} \in B_{X}$ and positive continuous measures $\mathrm{d}_{\mu_{n}}$ such that

$$
\begin{aligned}
& \mathfrak{L}=\otimes_{n}\left\{L_{2}\left(X_{n}, \mathrm{~d} \mu_{n}\right) \otimes \mathfrak{M}_{n}\right\} \\
& \mathrm{P}(Y)=\otimes_{n}\left\{\mathrm{P}_{n}\left(X_{n} \cap Y\right) \otimes 1\right\}
\end{aligned}
$$

where $\mathfrak{M}_{n}$ are Hilbert spaces of distinct dimensions and $\mathrm{P}_{n}(Z)$ is a multiplication operator of the characteristic function of $Z$.

Proof. First, we find a vector $\chi$ such that $(\chi, \mathrm{P}(Y) \chi)>0$ for every $Y \in B_{X}, \notin N_{X}^{\mathrm{E}}$. For this purpose, take arbitrary unit vector $\Psi_{1}$ and find the largest projection $\mathrm{P}\left(Y_{1}\right)$ such that $\mathrm{P}\left(Y_{1}\right) \Psi_{1}=0$. Next pick up a unit vector $\Psi_{2}$ from $\mathrm{P}\left(Y_{1}\right) \mathcal{R}$ and find largest projection $\mathrm{P}\left(Y_{2}\right)$ such that $\mathrm{P}\left(Y_{2}\right) \Psi_{1}=\mathrm{P}\left(Y_{2}\right) \Psi_{2}=0$. Continuing in this way by a transfinite induction, we have a strictly increasing sequence of projections $1-\mathrm{P}\left(Y_{\omega}\right)$ which exhausts $\&: \sum_{\alpha<\alpha_{0}} \mathrm{P}\left(Y_{\omega}\right)\left(1-\mathrm{P}\left(Y_{\omega+1}\right)\right)=1$. $\left(Y_{0} \equiv X\right.$.) By the countable chain condition, $\alpha_{0}$ is a countable ordinal. By construction, $\mathrm{P}(Y) \Psi_{\alpha}=0$ for all $\alpha<\alpha_{0}$ implies $\mathrm{P}(Y)=0$. Let $\alpha \rightarrow n(\alpha)$ be a one to one mapping of the ordinals $\alpha<\alpha_{0}$ to natural numbers. Then $\chi=\sum n(\alpha)^{-1} \Psi_{\alpha}$ has the desired property.

To find a decomposition of the required type, we construct 
$\left(\chi_{\alpha}, Z_{\alpha}, \mathbb{R}_{\alpha}\right)$ for every ordinals $\alpha<\alpha_{0}$ by a transfinite induction in such a way that (1) $\mathfrak{L}=\oplus \mathfrak{R}_{\alpha},(2)\left\{\mathrm{P}(Y) \chi_{\alpha} ; Y \in B_{X}\right\}$ generates $\mathfrak{E}_{\alpha}$, (3) $\mathrm{P}\left(Z_{\alpha}\right)$ is the largest projection of the form $\mathrm{P}(Y)$, vanishing identically on $\left\{\bigoplus_{\beta<\alpha} \mathcal{R}_{\beta}\right\}^{\perp}$, (4) $\left(\chi_{\alpha}, \mathrm{P}(Y) \chi_{\omega}\right)>0$ if $Y-Z_{\alpha} \notin N_{X}^{\mathrm{E}}$. Note that $\mathfrak{R}_{\alpha} \perp \mathfrak{R}_{\beta}$ for $\alpha \neq \beta$ and (3) imply $\mathrm{P}\left(Z_{\beta}\right) \leqq \mathrm{P}\left(Z_{\alpha}\right)$ for $\beta<\alpha$ and $\mathrm{P}\left(Z_{\alpha}\right) \mathfrak{R}_{\alpha}$ $=0$. For the construction, assume $\left(\chi_{\beta}, Z_{\beta}, \mathfrak{Q}_{\beta}\right)$ be given for $\beta<\alpha$. Define $\mathrm{P}\left(Z_{\alpha}\right)$ by (3). Use the previous construction of $\chi$ to define $\chi_{\alpha}$ as a unit vector in $\left\{\bigoplus_{\beta<\alpha} \mathfrak{R}_{\beta}\right\}^{\perp}$ satisfying (4). Define $\mathfrak{R}_{\alpha}$ by (2). This

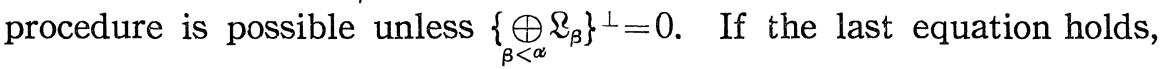
we set $\alpha=\alpha_{0}$ and we have (1).

To reach the form of (4.3) and (4.4), define $\mathrm{P}\left(Y_{\alpha}\right) \equiv \mathrm{P}\left(Z_{\omega}\right)-\underset{\beta<\infty}{\vee}$ $\mathrm{P}\left(Z_{\beta}\right)$. By the complete Boolean property of $B_{X}^{\mathrm{E}}, Y_{\alpha} \in B_{X}$ exists and by the countable chain condition, $\mathrm{P}\left(Y_{\omega}\right) \neq 0$ only for a countable number of $\alpha=\alpha_{n}, n=1,2, \cdots$. Set $X_{n}=X_{\alpha_{n}}$. We have $\sum \mathrm{P}\left(X_{n}\right)=1$, $\mathrm{P}\left(X_{n}\right) \mathrm{P}\left(X_{m}\right)=0$ for $n \neq m$, and $\mathfrak{L}=\oplus \hat{\mathfrak{Q}}_{n}, \hat{\mathfrak{Q}}_{n}=\mathrm{P}\left(X_{n}\right) \mathbb{R}$. Because of the property (4), $\mathrm{P}(Y) \chi_{\beta}^{n} \neq 0$ for $Y \subset X_{n}, Y \notin N_{X}^{\mathrm{E}}$ where we define $\chi_{\beta}^{n} \equiv$ $\mathrm{P}\left(X_{n}\right) \chi_{\beta}$ for $\beta<\alpha_{n}$. We have $\mathfrak{R}_{n}=\oplus \mathbb{R}_{\beta}^{n}$ where $\mathfrak{R}_{\beta}^{n}$ is generated by $\mathrm{P}(Y) \chi_{\beta}^{n}$. Since two measures $\left(X_{1}^{n}, \mathrm{P}(Y) \chi_{1}^{n}\right)$ and $\left(X_{\beta}^{n}, \mathrm{P}(Y) \chi_{\beta}^{n}\right)$ are equivalent, we have derivatives of one with respect to the other: $A(x)=\left(\chi_{1}^{n}, \mathrm{P}(\mathrm{d} x) \chi_{1}^{n}\right) /\left(\chi_{\beta}^{n}, \mathrm{P}(\mathrm{d} x) \chi_{\beta}^{n}\right)$. Then $\varphi_{\beta}^{n} \equiv \int A(x)^{1 / 2} \mathrm{P}(\mathrm{d} x) \chi_{\beta}^{n}$ satisfies $\left(\varphi_{\beta}^{n}, \mathrm{P}(Y) \varphi_{\beta}^{n}\right)=\left(\varphi_{1}^{n}, \mathrm{P}(Y) \phi_{1}^{n}\right)$. Since $\chi_{\beta}^{n}=\int A(x)^{-1 / 2} \mathrm{P}(\mathrm{d} x) \varphi_{\beta}^{n}, \mathfrak{R}_{\beta}^{n}$ is generated by $\mathrm{P}(Y) \varphi_{\beta}^{n}$ and $\mathrm{P}(Y)$ on all $\mathbb{R}_{\beta}^{n}$ with $n$ fixed are unitarily equivalent. Writing $\mu_{n}(Y)=\left(\chi_{1}^{n}, \mathrm{P}(Y) \chi_{1}^{n}\right)$ and $\mathfrak{R}_{\beta}^{n}=\mathbb{R}_{2}\left(X_{n}, \mathrm{~d} \mu_{n}\right) \otimes e_{\beta}$, $\mathfrak{M}_{n}=\bigoplus_{\beta<\alpha_{n}}\left\{c e_{\beta}\right\}, \varphi_{\beta}^{n}=1 \otimes e_{\beta}\left(\left\|e_{\beta}\right\|=1\right)$, we have (4.3) and (4.4).

If dimensions of some $\mathfrak{M}$ 's coincide, we lump together those $X_{n}$ for which $\mathfrak{M}_{n}$ has the same dimension and obtain (4.3) and (4.4) where $\mathfrak{M}$ 's have distinct dimensions.

Q.E.D.

Next we analyze a product unitary operator.

Lemma 4.2. A most general unitary product operator in a continuous tensor product is given by

$$
\mathrm{W}(c, \phi, Q)=e^{-\frac{1}{2}(\phi, \phi)+i c} \mathrm{~T}(-Q \phi, Q, \phi)
$$

where $Q$ is a unitary operator on $\&$, commuting with all $\mathrm{P}(Y), Y \in B_{X}$, $\phi$ is a vector in $\mathcal{R}, c$ is a real number and $\mathrm{T}(\psi, Q, \phi)$ is defined in Theorem 5.3 of ref. [3]. It satisfies 


$$
\begin{aligned}
& \mathrm{W}\left(c_{1}, \phi_{1}, Q_{1}\right) \mathrm{W}\left(c_{2}, \phi_{2}, Q_{2}\right) \\
& \quad=\mathrm{W}\left(c_{1}+c_{2}-\operatorname{Im}\left(\phi_{1}, Q_{2} \phi_{2}\right), Q_{2}^{*} \phi_{1}+\phi_{2}, Q_{1} Q_{2}\right) \\
& \mathrm{W}(c, \phi, Q) \exp \Psi=e^{-\frac{1}{2}(\phi, \phi)+i c+(\phi, \Psi)} \exp Q(\Psi-\phi) \\
& (\Omega, \mathrm{W}(c, \phi, Q) \Omega)=e^{-\frac{1}{2}(\phi, \phi)+i c} .
\end{aligned}
$$

Proof. The general form of bounded product operator is given by $k \mathrm{~T}(\psi, Q, \phi)$ where $k$ is a number, $Q$ is a bounded operator on $\mathbb{Q}$ with $\|Q\| \leq 1$, commuting with all $\mathrm{P}(Y), \psi$ and $\phi$ are vectors in $\&$ and $\phi+Q^{*} \psi$ is in the domain of $\left(1-Q^{*} Q\right)^{-1 / 2}$. The last condition is equivalent to the requirement that $\left.\mathrm{T}(\psi, Q, \phi)^{*} \mathrm{~T} / \psi, Q, \phi\right)$ be a bounded operator and is automatically satisfied if the unitarity requirement is satisfied. The latter is given by

$$
\begin{aligned}
& 1=|k|^{2} \mathrm{~T}(\psi, Q, \phi)^{*} \mathrm{~T}(\psi, Q, \phi)=|k|^{2} \mathrm{~T}\left(\phi+Q^{*} \psi, Q^{*} Q, \phi+Q^{*} \psi\right) e^{\left(\psi^{\prime}, \psi\right)} \\
& 1=|k|^{2} \mathrm{~T}(\psi, Q, \phi) \mathrm{T}(\psi, Q, \phi)^{*}=|k|^{2} \mathrm{~T}\left(\psi+Q \phi, Q Q^{*}, \psi+Q \phi\right) e^{(\phi, \phi)} .
\end{aligned}
$$

Since $k^{\prime} \mathrm{T}\left(\psi^{\prime}, Q^{\prime}, \phi^{\prime}\right)=1$ implies $k^{\prime}=1, \psi^{\prime}=\phi^{\prime}=0, Q^{\prime}=1$, we obtain the following necessary and sufficient condition for the unitarity:

$$
Q Q^{*}=Q^{*} Q=1, \quad \psi=-Q \phi, \quad|k|^{2}=e^{-(\phi, p)}
$$

The rest of the lemma follows from ref. [3].

(In terms of annihilation and creation operators, $T(\psi, Q, \phi)$ can be written formally as

$$
\left.\mathrm{T}(\psi, Q, \phi)=e^{\left(\mathrm{a}^{2} \psi\right)} e^{\left(\mathrm{d}^{*} \log Q \mathrm{a}\right)} e^{(\phi, \mathrm{a})} .\right)
$$

Lemma 4.3. If $\lim _{n \rightarrow \infty} \mathrm{W}\left(c_{n}, \phi_{n}, Q_{n}\right)=\mathrm{W}(c, \phi, Q)$ holds in the weak operator topology, then $\lim \exp i\left(c_{n}-c\right)=1, \lim \left\|\phi_{n}-\phi\right\|=0$, and $\lim Q_{n}=Q$ in the strong operator topology.

Proof. From (4.7), we have

$$
\left(e^{\Phi}, \mathrm{W}(c, \phi, Q) e^{\Psi}\right)=e^{-\frac{1}{2}(\phi,(h)+i c+(\phi, \Psi)-(\Phi, Q \phi)+(\Phi, Q \Psi)} .
$$

By setting $\Psi=\Phi=0$ and separating the absolute value and phase, we have

$$
\begin{aligned}
& \lim \left\|\phi_{n}\right\|^{2}=\|\phi\|^{2} \\
& \lim \exp i\left(c_{n}-c\right)=1 .
\end{aligned}
$$

By setting $\Phi=0$, taking sufficiently small $\Psi$ so that $\left|\left(\phi_{n}, \Psi\right)\right|<\pi$, 
$|(\phi, \Psi)|<\pi$ and using (4.10) and (4.11), we obtain

$$
\lim \left(\phi_{n}, \Psi\right)=(\phi, \Psi) \text {. }
$$

Together with (4.10), this implies $\lim \left\|\phi_{n}-\phi\right\|=0$. By setting $\Psi=0$ and using small $\Phi$, we have

$$
\lim \left(\Phi, Q_{n} \phi_{n}\right)=(\Phi, Q \phi) .
$$

Finally, by using small $\Phi$ and $\Psi$, and substituting previous results, we have

$$
\lim \left(\Phi, Q_{n} \Psi\right)=(\Phi, Q \Psi) .
$$

Because $Q_{n}$ and $Q$ are unitary, this implies $\lim Q_{n}=Q$ in the strong sense.

Q.E.D.

We now concentrate our attention to the subgroup $\mathrm{C}_{p}(G, X)$ of $\mathrm{C}(G, X)$ consisting of elements $g^{x(Y)}$ where $g \in G$ which is considered as a constant element of $\mathrm{C}(G, X)$ in the notation $g^{x(Y)}$ and $\chi(Y)$ is a characteristic function of $Y \in B_{X}^{\mathrm{E}}$. For each fixed $Y$, we have a continuous unitary representation of $G$

$$
g \in G \rightarrow \mathrm{U}\left(g^{\mathrm{x}(Y)}\right)=\mathrm{W}(c(g, Y), \phi(g, Y), Q(g, Y))
$$

where the continuity follows from Lemma 2.4. The relations among quantities with varying $Y$ are given by

$$
\begin{aligned}
& Q(g, Y)=\mathrm{P}(Y) Q(g, X)+1-\mathrm{P}(Y), \\
& \phi(g, Y)=\mathrm{P}(Y) \phi(g, X) .
\end{aligned}
$$

From

(4. 18) $\mathrm{E}\left(g^{x(Y)}\right)=\exp \left\{-\frac{1}{2}(\phi(g, X), \mathrm{P}(Y) \phi(g, X))+i c(g, Y)\right\}$

we have

$$
e^{i c(g, Y)}=\prod_{n} e^{i c\left(g, Y_{n}\right)}
$$

where $\left\{Y_{n}\right\}$ is a countable partition of $Y$ and the product must converge irrespective of the ordering.

Theorem 4.4. (4.15) gives a continuous unitary representation of $G$ for a fixed $Y$, if and only if (1) $Q(g, Y)$ is a continuous unitary representation of $G$, (2) $g \rightarrow \phi(g, Y)$ is a continuous mapping satisfying 


$$
\begin{gathered}
\phi(1, Y)=0 \\
Q\left(g_{2}, Y\right)^{*} \phi\left(g_{1}, Y\right)=\phi\left(g_{1} g_{2}, Y\right)-\phi\left(g_{2}, Y\right)
\end{gathered}
$$

and (3) $g \rightarrow e^{i c(g, Y)}$ is a continuous mapping satisfying

$$
\begin{gathered}
\exp i\left\{c\left(g_{1}, Y\right)+c\left(g_{2}, Y\right)-c\left(g_{1} g_{2}, Y\right)\right. \\
\left.\quad+\operatorname{Im}\left[\phi\left(g_{1}, Y\right), \phi\left(g_{2}^{-1}, Y\right)\right]\right\}=1 .
\end{gathered}
$$

If $\mathfrak{S}$ is spanned by $\mathrm{U}\left(g^{x(Y)}\right) \Omega$, then $\{\phi(g, Y)\}$ must span $\mathcal{L}$. If $\{\phi(g, Y)\}$ spans $\mathbb{R}$, then spaces $\mathfrak{M}_{n}$ in (4.3) are separable.

Proof. The first half is an immediate consequence of (4.6) and Lemma 4.3. ((4.20) follows from (4.21).) From (4.7) and (4.21), $\mathrm{U}\left(g^{x(Y)}\right) \Omega$ is a multiple of

$$
\exp -Q(g, Y) \phi(g, Y)=\exp \phi\left(g^{-1}, Y\right)
$$

which is in $\exp \mathfrak{Q}_{1}$ where $\mathfrak{Q}_{1}$ is the space spanned by all $\phi(g, Y)$. Therefore $\mathbb{R}_{1}=\mathcal{Q}$ is necessary in order that $\left\{\mathrm{U}\left(g^{x(Y)}\right) \Omega\right\}$ spans $e^{\mathbb{R}}$. Finally, to prove the separability of $\mathfrak{M}_{n}$, we note that $G$ is separable and hence has a countable dense set $\left\{g_{n}\right\}$. By continuity, $\left\{\phi\left(g_{n}, Y\right)\right\}$ generates \&. Let $E_{N}$ be the projection on the subspace spanned by $\phi\left(g_{n}, Y\right), n=1, \cdots, N, Y \in B_{X} . \quad E_{n}$ commutes with $\mathrm{P}(Y)$ by construction. Let $\mathfrak{Q}_{n}$ be the subspace generated by $\left(1-E_{n-1}\right) \phi\left(g_{n}, Y\right)$. Then $\mathfrak{Q}=\oplus \mathfrak{Q}_{n}$ and each $\mathfrak{R}_{n}$ is cyclic for $\{\mathrm{P}(Y)\}$. Let $\mathrm{P}\left(Y_{n}\right)$ be the largest $\mathrm{P}(Y)$ vanishing on $\mathfrak{Q}_{n}$. ( $Y_{n}$ defined up to a set in $\left.N_{X}^{\mathrm{E}}.\right)$ Define $\mathrm{P}_{m r} \equiv \sum \prod_{i=1}^{m} \mathrm{P}\left(Y_{i}^{(\mathrm{c})}\right)$ where (c) indicates $Y_{i}$ or $Y_{i}^{\mathrm{c}}$ and the summation runs over all possibilities such that the number of $Y_{i}^{\mathrm{c}}$ is exactly $r$. Define $\chi_{r}=\sum_{m \geqq r} m^{-1} \mathrm{P}_{m r}\left(1-E_{m-1}\right) \phi\left(g_{m}, X\right)$. This has the property of $\chi_{\beta}$ in Lemma 4.1 except some of $\chi_{r}$ here can be 0 . Since its total number is countable, $\operatorname{dim} \mathfrak{M}_{n}$ is finite or countably infinite. Q.E.D.

Definition 4.5. Let $\mathfrak{S}$ be a Hilbert space, $G$ be a group, and $Q(g), g \in G$ be a continuous unitary representation of $G$ on $\mathfrak{S}$. An S-valued continuous function $\phi(g)$ of $g \in G$ is called a cocycle of first order if it satisfies

$$
\phi\left(g_{2}\right)-\phi\left(g_{1} g_{2}\right)+Q\left(g_{2}\right) * \phi\left(g_{1}\right)=0
$$

The set of first order cocycles is denoted by $Z^{1}(G, \mathfrak{S})$. If 


$$
\phi(g)=\left(Q(g)^{*}-1\right) \Omega
$$

for an $\Omega \in \mathfrak{C}$, then $\phi$ is called a coboundary. They are denoted by $B^{1}(G, \mathfrak{S})$.

We shall study $\phi(g, Y)$ in a separate section. The representating operator for a general element of $\mathrm{C}(G, X)$ can be obtained from that of $\mathrm{C}_{p}(G, X)$ if we introduce the following additional continuity assumption.

Definition 4.6. The functional $\mathrm{E}(\mathrm{g})$ is called uniformly continuous if it is continuous with respect to the uniform topology on $\mathrm{C}(G, X)$. The uniform topology on $\mathrm{C}(G, X)$ is defined by neighbourhoods $N_{\mathfrak{N}}(\mathrm{g})=\left\{\mathrm{g}^{\prime} ; \mathrm{g}^{\prime}(x) \mathrm{g}(x)^{-1} \subset \mathfrak{N}\right.$ for all $\left.x\right\}$ where $\mathfrak{N}$ is a neighbourhood of 1 in $G$.

Theorem 4.7. An expectation functional $\mathrm{E}(\mathrm{g})$ is uniformly continuous if and only if the representation $\mathrm{U}(\mathrm{g})$ canonically associated with $\mathrm{E}$ is strongly continuous with respect to the uniform topology of $\mathrm{C}(G, X)$.

Proof. The if part is obvious. For the only if part, we note that given a compact $K$ in $G$ and an open neighbourhood $\mathfrak{R}$ of 1 in $G$, there exists a neighbourhood $\mathfrak{N}^{\prime}$ of 1 such that $\left\{x \mathfrak{N}^{\prime} x^{-1} ; x \in K\right\}$ $\subset \mathfrak{N}$. (Take a compact neighbourhood $\mathfrak{N}_{x}$ of 1 for each $x \in K$ such that $x \Re_{x} x^{-1} \subset \mathfrak{N}$. Then consider the set $K_{x}$ of all $y \in K$ satisfying $y \mathfrak{N}_{x} y^{-1} \subset \mathfrak{R} . \quad K_{x}$ is an open covering of $K$ and hence there exists a finite number of $K_{x}$ covering $K$. Take $\mathfrak{N}^{\prime}$ to be the intersection of $\mathfrak{N}_{x}$ for the finite number of $x$.) Hence if $\mathrm{E}(\mathrm{g})$ is uniformly continuous, then $\mathrm{E}\left(\mathrm{g}_{1} g g_{2}\right)$ for a fixed $\mathrm{g}_{1}$ and $\mathrm{g}_{2}$ is continuous in $\mathrm{g}$. From this $\mathrm{U}(\mathrm{g})$ is weakly continuous on a dense set. Since it is unitary, it is strongly continuous.

Q.E.D.

\section{§. Form of Expectation Functional}

Theorem 5.1. Let $B_{X}^{\mathrm{E}}$ be a continuous complete Boolean algebra of equivalence classes of subsets of $X$ satisfying the countable chain condition and let $F(g, Y)$ be a complex valued function of $g \in G$ and $Y \in B_{X}^{\mathrm{E}}$ satisfying the following condition;

(1) $F\left(g_{i} g_{j}^{-1}, Y\right)-F\left(g_{i}, Y\right)-F\left(g_{j}^{-1}, Y\right)=H\left(g_{i}, g_{j} ; Y\right)(\bmod 2 \pi i)$ 
(2) $H\left(g_{i}, g_{j} ; Y\right)$ is continuous in $g_{i}$ and $g_{j}$ for each $Y \in B_{X}^{\mathrm{E}}$.

(3) $H(1, g ; Y)=H(g, 1 ; Y)=0$

(4) $Y \rightarrow H\left(g_{i}, g_{j} ; Y\right)$ is complex finite measure on the complete Boolean algebra $B_{X}^{\mathrm{E}}$ for each fixed $g_{i}$ and $g_{j}$.

(5) $H\left(g_{i}, g_{j} ; Y\right)$ is a positive semidefinite matrix for any fixed $g_{i}$, $i=1, \cdots, N$, and $Y$.

(6) $\exp F(g, Y)$ is continuous in $g$.

(7) If $\left\{Y_{i}\right\}$ is a countable partition of $Y, \exp F(g, Y)=\prod_{i} \exp F\left(g, Y_{i}\right)$.

(8) $\operatorname{Re} F(g, Y)=\operatorname{Re} F\left(g^{-1}, Y\right)$.

Then there exists a continuous tensor product $\mathscr{E}=e^{\mathfrak{Q}}, \Omega=e^{0}$ and a continuous unitary representation $g^{x} \rightarrow \mathrm{U}\left(g^{x}\right)$ of the subgroup $\mathrm{C}_{p}(G, X)$ such that

$$
\mathrm{E}\left(g^{\chi(Y)}\right)=\left(\Omega, \mathrm{U}\left(g^{\chi(Y)}\right) \Omega\right)=e^{F(\mathcal{g}, Y)} .
$$

Conversely, any separable $\sigma$-factorizable functional over $\mathrm{C}(G, X)$ with no discrete spectrum is of this form when restricted to $\mathrm{C}_{p}(G, X)$.

Proof. We first prove the converse part. Let $\mathrm{U}\left(g^{x(Y)}\right)$ be given by (4.15) where $Q, \phi$ and $c$ satisfy (1), (2), (3) of Theorem 4.4 and are $\sigma$-additive in $Y$, mod. $2 \pi i$ for $c$. We compute $\mathrm{E}\left(g^{\mathrm{x}(Y)}\right) \equiv$ $\left(\Omega, \mathrm{U}\left(g^{x(Y)}\right) \Omega\right)$ in terms of $c(g, Y), Q(g) \equiv Q(g, X), \phi(g) \equiv \phi(g, X)$ and $\mathrm{P}(Y)$. We have

$$
\mathrm{E}\left(g^{\mathrm{x}(Y)}\right)=e^{-\frac{1}{2}(\phi(g), \mathrm{P}(Y) \nprec(g))+i c(\boldsymbol{g}, Y)}
$$

and hence define

$$
F(g, Y)=-\frac{1}{2}(\phi(g), \mathrm{P}(Y) \phi(g))+i c(g, Y) .
$$

$e^{F(g, Y)}$ is continuous in $g$ for fixed $Y$ by Lemma 2.4. We also have $e^{F(1, Y)}=\mathrm{E}(1)=1$. Because we have a freedom of adding an integral multiple of $2 \pi$ to $c(g, Y)$, we set $c(1, Y)=0$. Then $F(1, Y)=0$. From (4.20) and (4.21), we have $\phi\left(g^{-1}, Y\right)=-Q(g, Y) \phi(g, Y)$. Hence the unitarity of $Q(g, Y)$ implies (8). Next we compute

$$
G\left(g_{i}, g_{j} ; Y\right) \equiv F\left(g_{i} g_{j}^{-1}, Y\right)-F\left(g_{i} Y\right)-F\left(g_{j}^{-1} Y\right) .
$$

From the unitarity of $Q(g)$ and equation (4.17), we have 
Hence

$$
\begin{aligned}
\left\|\mathrm{P}(Y)\left[\phi\left(g_{1} g_{2}\right)-\phi\left(g_{2}\right)\right]\right\|^{2} & =\left\|\mathrm{P}(Y) Q\left(g_{2}\right) * \phi\left(g_{1}\right)\right\|^{2} \\
& =\left\|Q\left(g_{2}\right) * \mathrm{P}(Y) \phi\left(g_{1}\right)\right\|^{2} \\
& =\left\|\mathrm{P}(Y) \phi\left(g_{1}\right)\right\|^{2}
\end{aligned}
$$

$$
\begin{aligned}
& -\left(\phi\left(g_{1}\right), \mathrm{P}(Y) \phi\left(g_{1}\right)\right)+\left(\phi\left(g_{1} g_{2}\right), \mathrm{P}(Y) \phi\left(g_{1} g_{2}\right)\right) \\
& +\left(\phi\left(g_{2}\right), \mathrm{P}(Y) \phi\left(g_{2}\right)\right)=2 \operatorname{Re}\left(\phi\left(g_{1} g_{2}\right), \mathrm{P}(Y) \phi\left(g_{2}\right)\right) .
\end{aligned}
$$

Setting $g_{2}=g_{j}, g_{1}=g_{i} g_{j}^{-1}$, we have

$$
\begin{gathered}
\operatorname{Re}\left[F\left(g_{i} g_{j}^{-1}, Y\right)-F\left(g_{i}, Y\right)-F\left(g_{j}, Y\right)\right] \\
=\operatorname{Re}\left(\phi\left(g_{i}\right), \mathrm{P}(Y) \phi\left(g_{j}\right)\right) .
\end{gathered}
$$

By setting $g_{i}=1$, we have $\operatorname{Re} F\left(g^{-1}, Y\right)=\operatorname{Re} F(g, Y)$. Hence

$$
\begin{gathered}
\operatorname{Re}\left[F\left(g_{i} g_{j}^{-1}, Y\right)-F\left(g_{i}, Y\right)-F\left(g_{j}^{-1}, Y\right)\right] \\
=\operatorname{Re}\left(\phi\left(g_{i}\right), \operatorname{P}(Y) \phi\left(g_{j}\right)\right) .
\end{gathered}
$$

Next we consider (4.22). We have

(5. 9) $\quad c\left(g_{1} g_{2}, Y\right)-c\left(g_{1}, Y\right)-c\left(g_{2}, Y\right)=\operatorname{Im}\left(\phi\left(g_{1}\right), \mathrm{P}(Y) \phi\left(g_{2}^{-1}\right)\right)$

modulo $2 \pi$. Hence, we obtain modulo $2 \pi$

$$
\begin{aligned}
\operatorname{Im}[ & {\left[F\left(g_{i} g_{j}^{-1}, Y\right)-F\left(g_{i}, Y\right)-F\left(g_{j}^{-1}, Y\right)\right] } \\
& =\operatorname{Im}\left(\phi\left(g_{i}\right), \mathrm{P}(Y) \phi\left(g_{j}\right)\right) .
\end{aligned}
$$

Combining (5.7) and (5.10), we have

(5. 11) $\quad F\left(g_{i} g_{j}^{-1}, Y\right)-F\left(g_{i}, Y\right)-F\left(g_{j}^{-1}, Y\right)=\left(\phi\left(g_{i}\right), \mathrm{P}(Y) \phi\left(g_{j}\right)\right)$

modulo $2 \pi i$. By taking $H\left(g_{i}, g_{j} ; Y\right)$ to be the right hand side, we have the properties (2)-(5) for $H$.

Now let $F$ and $H$ be given. We introduce the free complex linear space $K_{0}$ over $B_{X}^{\mathrm{E}} \times G$, denoting a general element by

$$
\sum_{n=1}^{N} c_{n} \Psi\left(Y_{n}, g_{n}\right), \quad Y_{n} \in B_{X}^{\mathrm{E}}, g_{n} \in G .
$$

We introduce an inner product by linearity and

$$
\left(\Psi\left(Y_{1}, g_{1}\right), \Psi\left(Y_{2}, g_{2}\right)\right)=H\left(g_{1}, g_{2} ; Y_{1} \cap Y_{2}\right) \text {. }
$$

After identifying all elements with 0 norm as 0 , we obtain a prehilbert space. We denote its completion by \&. We define $\phi(g)$, $c(g, Y), \mathrm{P}(Y)$ and $Q(g)$ by 


$$
\begin{aligned}
& \phi(g)=\Psi(X, g), \quad c(g, Y)=\operatorname{Im} F(g, Y) \\
& \mathrm{P}(Y) \Psi\left(Y^{\prime}, g\right)=\Psi\left(Y \cap Y^{\prime}, g\right) \\
& Q(g) \Psi\left(Y, g^{\prime}\right)=\Psi\left(Y, g^{\prime} g^{-1}\right)-\Psi\left(Y, g^{-1}\right) .
\end{aligned}
$$

First we note that $\phi(1)=0$, because ||$\Psi(X, 1) \|^{2}=H(1,1 ; X)=0$. By linearity, we want to extend $\mathrm{P}(Y)$ and $Q(g)$ to a dense subset. Because of (4) and (5), $\mathrm{P}(Y)$ brings 0 always to 0 and hence the linear extension is possible. Further (4) and (5) imply that $\mathrm{P}(Y)$ is bounded. From the definition, we see $\mathrm{P}(Y)^{*}=\mathrm{P}(Y)$ and $\mathrm{P}(Y)^{2}=\mathrm{P}(Y)$. Namely $\mathrm{P}(Y)$ is a projection. (4) then tells us that $Y \rightarrow \mathrm{P}(Y)$ is a projection valued measure.

From the continuity of $H\left(g_{i}, g_{j} ; Y\right)$ in $g_{i}$ and $g_{j}$, we see that $\Psi(Y, g)$ is strongly continuous in $g$. Hence $\phi(g)$ and $Q(g)$ are strongly continuous in $g$.

For $Q(g)$, the structure of $H$ and the definition (5.16) of $Q$ imply

$$
\begin{aligned}
&\left(Q(g) \Psi\left(Y_{2}, g_{2}\right), Q(g) \Psi\left(Y_{1}, g_{1}\right)\right) \\
&=\left(\Psi\left(Y_{2}, g_{2} g^{-1}\right)-\Psi\left(Y_{2}, g^{-1}\right), \Psi\left(Y_{1}, g_{1} g^{-1}\right)-\Psi\left(Y_{1}, g^{-1}\right)\right) \\
&= H\left(g_{2} g^{-1}, g_{1} g^{-1} ; Y_{2} \cap Y_{1}\right)-H\left(g^{-1}, g_{1} g^{-1} ; Y_{2} \cap Y_{1}\right) \\
&-H\left(g_{2} g^{-1}, g^{-1} ; Y_{2} \cap Y_{1}\right)+H\left(g^{-1}, g^{-1} ; Y_{2} \cap Y_{1}\right) \\
&= F\left(g_{2} g_{1}^{-1}, Y_{2} \cap Y_{1}\right)-F\left(g_{1}^{-1}, Y_{2} \cap Y_{1}\right)-F\left(g_{2}, Y_{2} \cap Y_{1}\right) \\
&=\left(\Psi\left(Y_{2}, g_{2}\right), \Psi\left(Y_{1} g_{1}\right)\right) .
\end{aligned}
$$

Here we have used $F(1, Y)=0$, which follows from (1) with $g_{i}=1$ and (3). (5.17) holds up to $2 n \pi i, n=0, \pm 1, \cdots$. However, the continuity of $Q(g)$ implies $n=0$. Hence $Q(g)$ can be extended to the whole space as an isometric operator by linearity and continuity. From the definition $Q\left(g_{1}\right) Q\left(g_{2}\right)=Q\left(g_{1} g_{2}\right)$ holds on a total set $\Psi(Y, g)$ and hence on all vectors. Since $Q(1)=1, Q\left(g^{-1}\right) Q(g)=Q(g) Q\left(g^{-1}\right)=1$. Hence $Q(g)^{-1}$ exists, which implies that $Q(g)$ is unitary. Further $Q(g)^{*}=Q\left(g^{-1}\right)$. By definition $Q(g)$ commutes with all $\mathrm{P}(Y)$. Since $\operatorname{Re} F(1, Y)=0$, (5.13), (5.15) and (8) imply $(\phi(g), \mathrm{P}(Y) \phi(g))=$ $-2 \operatorname{Re} F(g, Y)$. If we define $\mathrm{U}\left(g^{x(Y)}\right)$ by (4.15), (4.16) and (4.17) with $\phi(g, X)=\phi(g), Q(g, X)=Q(g)$, then the equation (5.1) is satisfied.

The function $c(g, Y)$ is $\sigma$-additive in $Y \bmod 2 \pi$ by (7). It satisfies (4.22) due to (5.14), (1), (5.13) and (5.15). It is continuous in $g$ due to (6). Thus $\mathrm{U}\left(g^{x(Y)}\right)$ is a continuous unitary representation of $\mathrm{C}_{p}(G, X)$.

Q.E.D. 
We remark that the absolute value part of $(7)$ is a consequence of other assumptions. We also note that $\operatorname{Im} F(g, Y)=-\operatorname{Im} F\left(g^{-1}, Y\right)$ $\bmod 2 \pi$ follows from (1) and (5).

In the rest of this section, we rederive the form of the functional $\mathrm{E}(\mathrm{g})$ in the preceding theorem by an elementary method under the assumption that the functional is given by

$$
\mathrm{E}(\mathrm{g})=\exp \int_{X} F(\mathrm{~g}(x)) \mathrm{d} \mu(x) .
$$

Here $F(g)$ is assumed to be continuous in $g, F(1)=0$ and $\mu$ is a continuous positive finite measure on $X$.

Lemma 5.2. Let $A_{i j}, i, j=1, \cdots, n$, be a matrix. The matrix $\left(\exp c A_{i j}\right)$ is positive semidefinite for all positive $c$ if and only if $A$ is hermitian and PAP is positive semidefinite where $P_{i j}=\delta_{i j}-\frac{1}{n}$.

Proof. (1) The sufficiency: We have

$$
\exp c A_{i j}=e^{\alpha}\left(e^{\beta_{i}}\right)^{*}\left(\exp c(P A P)_{i j}\right)\left(e^{\beta_{j}}\right),
$$

where

$$
\begin{aligned}
& \alpha=-\frac{c}{n^{2}} \sum_{i j} A_{i j} \text { (real), } \\
& \beta_{j}=\frac{c}{n} \sum_{i} A_{i j} .
\end{aligned}
$$

Hence

$$
\sum x_{i}^{*}\left(\exp c A_{i j}\right) x_{j}=e^{\alpha} \sum y_{i}^{*}\left(\exp c(P A P)_{i j}\right) y_{j}
$$

where

$$
y_{j}=x_{j} e^{\beta_{j}} .
$$

By Lemma 8.2 of [3], the positive semidefiniteness of $(P A P)_{i j}$ implies the same for $\exp c(P A P)_{i j}$. Therefore we have the positive semidefiniteness of $\exp c A_{i j}$ from (5.22).

(2) The necessity: Let $P x=x$, namely $\sum x_{i}=0$. Then

$$
\sum_{i j} x_{i}^{*} A_{i j} x_{j}=\lim _{c \rightarrow 0} c^{-1} \sum_{i j} x_{i}^{*} e^{c A_{i j}} x_{j} \geq 0 .
$$

Since $P$ is a projection, we have the positive semidefiniteness of $P A P$.

It follows that $B_{i j} \equiv \exp c(P A P)_{i j}$ is hermitian. Since $f(c) \equiv$ 
$\sum_{i j} \exp c A_{i j}$ must be real, $\alpha=-\left(c / n^{2}\right) f^{\prime}(0)$ is real. Since $\exp c A_{i j}=$ $B_{i j} \exp \left(\alpha+\gamma_{i}+\beta_{j}\right)$ is hermitian, where $\gamma_{i} \equiv(c / n) \sum_{j} A_{i j} ;$ we have $\gamma_{j}^{*}=\beta_{j}+2 n \pi i$. From the continuity in $c$, we have $n=0$. Hence $A_{i j}=\alpha+\gamma_{i}+\beta_{j}+(P A P)_{i j}$ is hermitian.

Q.E.D.

We now consider

$$
\mathrm{E}\left(g^{x(Y)}\right)=\exp F(g) \mu(Y) .
$$

By the Lemma 5.2, the positivity condition for $\mathrm{E}$ implies

$$
\sum_{i, j} x^{*} F\left(g_{i} g_{j}^{-1}\right) x_{j} \geq 0
$$

whenever $\sum x_{i}=0$. Let $i, j=0,1, \cdots, n, g_{0}=1, x_{0}=-\sum_{i=1}^{n} x_{i}$. Then we have

$$
\begin{gathered}
\sum_{i, j=1}^{n} x_{i}^{*} F\left(g_{i} g_{j}^{-1}\right) x_{j}-\left(\sum_{i=1}^{n} x_{i}^{*}\right) \sum_{i=1}^{n} F\left(g_{j}^{-1}\right) x_{j} \\
-\sum_{i=1}^{n} x_{i}^{*} F\left(g_{i}\right)\left(\sum_{i=1}^{n} x_{i}\right) \geq 0 .
\end{gathered}
$$

Namely $F\left(g_{i} g_{j}^{-1}\right)-F\left(g_{i}\right)-F\left(g_{j}^{-1}\right)$ is positive semidefinite.

Conversely, assume that $F\left(g_{i} g_{j}^{-1}, x\right)-F\left(g_{i}, x\right)-F\left(g_{j}^{-1}, x\right), i, j=$ $1, \cdots, n$ is positive semidefinite for any $\left\{g_{i}\right\}, n$ and $x$. By setting $n=1, x_{1}=1$, we have $F(1, x)=0$. Let $\mu$ be a finite positive measure on $X$. Then

$$
F(\mathrm{~g}) \equiv \int F(\mathrm{~g}(x), x) \mathrm{d} \mu(x), \quad \mathrm{g} \in \mathrm{C}(X, G)
$$

have the property that $H\left(\mathrm{~g}_{i}, \mathrm{~g}_{j}\right) \equiv F\left(\mathrm{~g}_{i} \mathrm{~g}_{j}^{-1}\right)-F\left(\mathrm{~g}_{i}\right)-F\left(\mathrm{~g}_{j}^{-1}\right)$ is positive semidefinite and $F(1)=0$. Then, for any $x_{i}$ satisfying $\sum x_{i}=0$, we have

$$
\sum x_{i}^{*} H\left(\mathrm{~g}_{i}, \mathrm{~g}_{j}\right) x_{j}=\sum x_{i}^{*} F\left(\mathrm{~g}_{i} \mathrm{~g}_{j}^{-1}\right) x_{j} \geq 0 .
$$

Hence $\mathrm{E}\left(\mathrm{g}_{i} \mathrm{~g}_{j}^{-1}\right)$ is positive semidefinite and $\mathrm{E}(1)=1$. In particular, if $F(g, x)$ is constant in $x$, we have the positivity for (5.18).

\section{§6. Standard Examples for $\phi(g, Y)$}

To analyze $\phi(g, Y)$, we introduce the following spaces.

Definition 6.1. Let $h(t)$ be a function of the class $\mathscr{D}$ such that its Fourier transform $\widehat{h}(\lambda)=\int e^{i t \lambda} h(t) \mathrm{d} t$ satisfies $\widehat{h}(0)=0,1>\widehat{h}(\lambda) \geq 0$, 
for $\lambda \neq 0$ and $\widehat{h}^{\prime \prime}(0) \neq 0$. Let $\mathfrak{I}_{i}$ be a linearly independent basis of $\mathrm{g}$, and

$$
\mathrm{K}=\sum \mathrm{K}\left(\mathfrak{I}_{i}\right), \mathrm{K}(\mathfrak{l})=1-\int Q\left(e^{t \mathfrak{I}}\right) h(t) d t .
$$

Let $\mathrm{K}=\int_{0}^{\infty} \lambda E(\mathrm{~d} \lambda)$ and $\mathrm{K}^{-1 / 2}$ be the inverse of the mapping $\mathrm{K}^{1 / 2}$ from $(1-E([0])) \&$ into \&.

The space $D^{ \pm}$is the range of $\mathrm{K}^{\mp 1 / 2}$ in $\mathfrak{Q}$ equipped with the topology induced by a new norm $\|\psi\|_{ \pm} \equiv\left\|\mathrm{K}^{ \pm 1 / 2} \psi\right\|$ and $\bar{D}^{+}$is the completion of $D^{+}$. The space $D_{0}$ is the largest subspace of $\&$ on which $Q(g)=1$ for all $g$.

Lemma 6.2. $D^{ \pm}$as a topological linear space does not depend on the choice of $h$ and $\left\{\mathrm{I}_{i}\right\} . D_{0}$ is the eigenspace belonging to the eigenvalue 0 of $\mathrm{K} .(Q(g)-1) \Psi$ for any $g \in G$ and $\Psi \in \mathcal{L}$ belongs to $D^{-}$and

$$
\|(Q(g)-1) \Psi\|_{-} \leq d(g)\|\Psi\|
$$

where $d(g)$ does not depend on $\Psi$. For every $\Psi,\|(Q(g)-1) \Psi\|_{-} \rightarrow 0$ as $g \rightarrow 1$.

Proof. We first characterize a vector in the range of $\mathrm{K}\left(\mathfrak{l}_{i}\right)^{1 / 2}$. Consider the spectral decomposition of one parameter family of unitary operators

$$
Q\left(e^{t\urcorner}\right)=\int e^{i t \lambda} \mathrm{d} E_{\mathrm{r}}(\lambda)
$$

Then

$$
\mathrm{K}(\mathfrak{l})=\int[1-\widetilde{h}(\lambda)] \mathrm{d} E_{\mathrm{r}}(\lambda) .
$$

By assumption $1-\widetilde{h}(\lambda) \geq 0$, where the equality holds only at $\lambda=0$, $1-\widehat{h}(\lambda) \rightarrow 1$ as $\lambda \rightarrow \infty$ and of order $\lambda^{2}$ at $\lambda=0$. Hence a vector $\psi$ is in the domain of $\mathrm{K}(\mathfrak{l})^{-1 / 2}$ if and only if $\int \lambda^{-2} \mathrm{~d}\left(\psi, E_{\mathfrak{l}}(\lambda) \psi\right)<\infty$. In particular $\left(Q\left(e^{t \mathfrak{I}}\right)-1\right) \psi$ is in the domain of $\mathrm{K}(\mathfrak{L})^{-1 / 2}$ because $\lambda^{-2}\left|e^{i \lambda t}-1\right|^{2}$ is a bounded function of $\lambda$. We also know that $K(\mathfrak{l}) \psi=0$ if and only if $Q\left(e^{t \mathfrak{l}}\right) \psi=\psi$ for all $t$. Next, since all $\mathrm{K}\left(\mathfrak{I}_{i}\right) \geq 0$, we have $\mathrm{K} \geq \mathrm{K}\left(\mathrm{I}_{i}\right)$.

Now we see that the eigenspace of $\mathrm{K}$ belonging to the eigenvalue 0 is the intersection of the same for $K\left(\mathrm{I}_{i}\right)$ and hence consists 
of $\psi$ such that $Q\left(e^{t \mathrm{I}_{i}}\right) \psi r=\psi$. Since $e^{t \mathrm{I}_{i}}$ generates $G$, we have proved the assertion on $D_{0}$.

Next we prove that $\left(Q\left(e^{t \mathrm{I}_{i}}\right)-1\right) \psi$ is in $D^{-}$. Since $\mathrm{K}\left(\mathrm{I}_{i}\right) \geq 0$, we have $(\varphi, \mathrm{K} \varphi) \geq\left(\varphi, \mathrm{K}\left(\mathbb{I}_{i}\right) \varphi\right)$ for any $\varphi$. Hence $\left\|\mathrm{K}\left(\mathfrak{I}_{i}\right)^{1 / 2} \mathrm{~K}^{-1 / 2} \varphi_{1}\right\|^{2} \leq\left\|\varphi_{1}\right\|^{2}$ where $\varphi_{1}=\mathrm{K}^{1 / 2} \varphi$. Hence $\mathrm{K}\left(\mathrm{I}_{i}\right)^{1 / 2} \mathrm{~K}^{-1 / 2}$ has a bounded closure with norm $\leqq 1$, which we denote by $R_{i}$. The domain of $R_{i}$ is $D_{0}^{\perp}$. If $T$ is a bounded linear operator and $S$ is a linear operator with a dense domain, then $(T S)^{*}=S^{*} T^{*}$ ([5] p. 297). Since the ranges of $\mathrm{K}\left(\mathfrak{I}_{i}\right)^{1 / 2}$ and $\mathrm{K}^{-1 / 2}$ are contained in $D_{0}^{\perp}$, we consider the restrictions $T=$ $\mathrm{K}\left(\mathrm{I}_{i}\right)^{1 / 2} \mid D_{0}^{\perp}$ and $S=\mathrm{K}^{-1 / 2} \mid D_{0}^{\perp}$ on the space $D_{0}^{\perp}$. Then

$$
R_{i}^{*} \varphi=(T S)^{*} \varphi=\mathrm{K}^{-1 / 2} \mathrm{~K}\left(\mathrm{I}_{i}\right)^{1 / 2} \varphi
$$

for $\varphi \in D_{0}^{\perp}$ where $R_{i}^{*}$ is the adjoint of $R_{i}$ on $D_{0}^{\perp}$. Therefore,

$$
\left\|\mathrm{K}^{-1 / 2} \mathrm{~K}\left(\mathrm{Y}_{i}\right)^{1 / 2} \phi\right\| \leq\|\varphi\|
$$

for $\varphi \in D_{0}^{\perp}$. Since $\mathrm{K}\left(\mathrm{I}_{i}\right)^{1 / 2} \varphi=0$ for $\varphi \in D_{0}$, we see that $\mathrm{K}\left(\mathrm{I}_{i}\right)^{1 / 2} \varphi$ for any $\varphi$ is in the domain of $\mathrm{K}^{-1 / 2}$ and the above inequality holds.

Since

$$
\rho(t)=\sup _{\lambda} 2(1-\cos t \lambda)(1-\widehat{h}(\lambda))^{-1}<\infty,
$$

$\varphi_{3}=\left[Q\left(e^{t t_{i}}\right)-1\right] \psi$ for any $\psi$ is in the domain of $\mathrm{K}\left(\mathfrak{I}_{i}\right)^{-1 / 2}$. Hence setting $\varphi_{2}=\mathrm{K}\left(\mathfrak{Y}_{i}\right)^{-1 / 2} \varphi_{3}$, we see that $\varphi_{3}$ is in the domain of $\mathrm{K}^{-1 / 2}$ and

$$
\left\|\left[Q\left(e^{t \mathfrak{t}_{i}}\right)-1\right] \psi\right\|\left\|_{-}^{2}=\right\| \mathrm{K}^{-1 / 2} \varphi_{3}\left\|^{2} \leq\right\| \mathrm{K}\left(\mathrm{I}_{i}\right)^{-1 / 2} \varphi_{3}\left\|^{2} \leq \rho(t)\right\| \psi \|^{2} .
$$

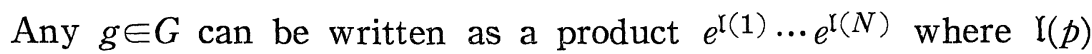
is of the form $t_{n} \mathfrak{l}_{i(p))}$ for some $i(p)$. Then, writing $g(p)=e^{\mathfrak{l}(p)} \cdots e^{\mathfrak{l}(N)}$ and $g(N+1)=1$, we have

$$
\begin{gathered}
(Q(g)-1) \psi=\sum_{p=1}^{N}\left\{Q\left(e^{\mathfrak{I}(p)}\right)-1\right\} Q(g(p+1)) \psi, \\
\|\{Q(g)-1\} \psi\|_{-} \leq\left\{\sum_{p=1}^{N} \rho\left(t_{p}\right)^{1 / 2}\right\}\|\psi\| .
\end{gathered}
$$

We thus have proved that $\{Q(g)-1\} \psi \in D_{-}$and the equation (6.2). Near $g=1$, we may take $i(p)=p$ (running over a basis) and $g \rightarrow 1$ is equivalent to $\left\{t_{p}\right\} \rightarrow 0$. As $t \rightarrow 0$ and $\psi_{s} \rightarrow \psi, \delta_{t}(\psi) \equiv \| \mathrm{K}\left(\mathfrak{I}_{i}\right)^{-1 / 2}\left[Q\left(e^{t I_{i}}\right)\right.$ $-1] \psi \| \rightarrow 0$ and $\delta_{t}\left(\psi_{s}\right) \leq \rho(t)\left\|\psi_{s}-\psi\right\|+\delta_{t}(\psi) \rightarrow 0$. Hence (6.7) implies $\|(Q(g)-1) \psi\|_{-} \rightarrow 0$ as $g \rightarrow 1$ for every $\psi$ in $\mathfrak{R}$.

Finally we prove that different choices of $\hat{h}$ and $\left\{\mathfrak{I}_{i}\right\}$ give the 
same $D^{ \pm}$. Let $\rho_{\nu}(t)$ and $\mathrm{K}_{\nu}(\mathfrak{l})$ be the $\rho$ and $\mathrm{K}(\mathfrak{l})$ with $\widehat{h}=\widetilde{h}_{\nu}, d_{\nu}(g)$ be the $d(g)$ with $\widehat{h}_{\nu}$ and $\left\{\mathfrak{l}_{i}^{\nu}\right\}, \mathrm{K}_{\nu}=\sum \mathrm{K}_{\nu}\left(\mathfrak{l}_{i}^{\nu}\right)$ where $\nu=1$ and 2. For $\varphi=\left\{Q\left(e^{t I}\right)-1\right\} \psi$, we have

$$
\left\|\mathrm{K}_{1}^{-1 / 2} \varphi\right\|^{\|} \leq d_{1}\left(e^{t \mathrm{l}}\right)\|\psi\| .
$$

Choose an $a$ satisfying $0<a<2 \pi / t$ and define $E_{a} \equiv E_{\mathrm{r}}([-a, a])$. Then $R \equiv\left(Q\left(e^{t l}\right)-1\right)^{-1} \mathrm{~K}_{2}(\mathfrak{l})^{1 / 2} E_{a}$ is bounded and hence $\|\psi\|=\left\|R \mathrm{~K}_{2}(\mathfrak{l})^{-1 / 2} \varphi\right\| \leq$ $i \mid R\|\| \mathrm{K}_{2}(\mathfrak{I})^{-1 / 2} \phi \|$ if $E_{a} \psi=\psi$ and $E_{0} \psi=0$. If $\psi$ runs over $\left(E_{a}-E_{0}\right) \&$, then $\varphi=\left(Q\left(e^{t \downarrow}\right)-1\right) \psi$ runs over $E_{a}$ times the domain of $\mathrm{K}_{2}(\mathfrak{l})^{-1 / 2}$. (Note that $E_{0} \mathbb{R}$ is orthogonal to the domain of $\mathrm{K}_{2}(\mathfrak{l})^{-1 / 2}$.) Hence we have

$$
\left\|\mathrm{K}_{1}^{-1 / 2} \mathrm{~K}_{2}(\mathfrak{l})^{1 / 2} \Phi\right\| \leq c(\mathfrak{l})\|\Phi\|
$$

for $\Phi=\mathrm{K}_{2}(\mathfrak{I})^{-1 / 2} \varphi$, which runs over $\left(E_{a}-E_{0}\right) \mathfrak{R}$. If $\Phi \in E_{0} \mathfrak{R}$, then the left hand side of (6.10) is 0 and hence (6.10) holds trivially.

Next consider $\psi$ in $\left(1-E_{a}\right)$ R. If $A$ is a closed operator and $\psi_{n}$ is a sequence of vectors in the domain of $A$ such that $\left\|A \psi_{n}\right\| \leq a$ for all $n$ and $\lim \psi_{n}=\psi$, then $\psi$ is also in the domain of $A$ and $\|A \psi\| \leq a$ due to the weak sequential compactness. If $\psi(t)$ is measureable in $t, \psi=\int \psi(t) \mathrm{d} t$ converges strongly, $\|A \psi(t)\| \leq a(t)$ and $\int a(t) \mathrm{d} t$ $<\infty$, then $\psi$ is in the domain of $A$ and $\|A \psi\| \leq \int a(t) \mathrm{d} t$. By using this, we integrate (6.9) relative to $h_{2}(t) \mathrm{d} t$ where $d(\mathfrak{l}) \equiv \int d_{1}\left(e^{t l}\right) h_{2}(t) \mathrm{d} t$ $<\infty$. We have $\left\|\mathrm{K}_{1}^{-1 / 2} \mathrm{~K}_{2}(\mathfrak{l}) \psi\right\| \leq d(\mathfrak{l})\|\psi\|$. If we set $\Phi=\mathrm{K}_{2}(\mathfrak{l})^{1 / 2} \psi, \Phi$ runs over $\left(1-E_{a}\right) \mathbb{R}$ when $\psi$ runs over $\left(1-E_{a}\right) \mathfrak{R}$ and $\|\psi\| \leq \| \mathrm{K}_{2}\left(\mathfrak{l}^{-1 / 2}\right.$ $\times\left(1-E_{a}\right)\|\| \Phi \|$. Hence (6.10) with a new constant $c(\mathfrak{l})$ holds also for such $\Phi$.

Thus we see that $\mathrm{K}_{1}^{-1 / 2} \mathrm{~K}_{2}(l)^{1 / 2}$ is a bounded operator. Taking adjoint, we see that

$$
|| \mathrm{K}_{2}(\mathfrak{l})^{1 / 2} \mathrm{~K}_{1}^{-1 / 2} \varphi\|\leq a(\mathfrak{l})\| \varphi \|
$$

for any $\varphi$ in the domain of $\mathrm{K}_{1}^{-1 / 2}$. Setting $\mathrm{K}_{1}^{-1 / 2} \varphi=\psi$, we have

$$
\left(\psi, \mathrm{K}_{2}(\mathfrak{l}) \psi\right) \leq a(\mathfrak{l})^{2}\left(\psi, \mathrm{K}_{1} \psi\right)
$$

where $\psi \in D_{0}^{\perp}$. By adding this equation for $\mathfrak{l}=\mathfrak{l}_{i}^{2}, i=1, \cdots$, we have

$$
\left(\psi, \mathrm{K}_{2} \psi\right) \leq a_{1}\left(\psi, \mathrm{K}_{1} \psi\right)
$$

for some constant $a_{1}$ and for any $\psi$ in the orthogonal complement 
of $D_{0}$. Since $D_{0}$ is the 0 eigenspace of both $\mathrm{K}_{1}$ and $\mathrm{K}_{2}$, an addition of vectors in $D_{0}$ to $\psi$ does not change the inequality (6.10) and we have $\mathrm{K}_{2} \leq a_{1} \mathrm{~K}_{1}$. Similarly, we have $\mathrm{K}_{1} \leq a_{2} \mathrm{~K}_{2}$. Hence $D^{-}$is the same for $\nu=1$ and 2. From $\mathrm{K}_{1} \leq a_{2} \mathrm{~K}_{2}$, we have

$$
\left\|\mathrm{K}_{1}^{1 / 2} \mathrm{~K}_{2}^{-1 / 2} \psi\right\|\left\|^{2} \leq a_{2}\right\| \psi \|^{2}
$$

for $\psi \in \mathrm{K}_{2}^{1 / 2} \Omega$. Since $\mathrm{K}_{2}^{1 / 2} \Omega$ span $D_{0}^{\perp}, \mathrm{K}_{1}^{1 / 2} \mathrm{~K}_{2}^{-1 / 2}$ is bounded on $D_{0}^{\perp}$.

Namely, $\left|\left(\mathrm{K}_{1}^{1 / 2} \varphi, \mathrm{K}_{2}^{-1 / 2} \psi\right)\right| \leq a_{2}^{1 / 2}\|\varphi\|\|\psi\|$ for any $\psi$ in the domain of $\mathrm{K}_{2}^{-1 / 2}$ and any $\varphi$. Hence $\left(\mathrm{K}_{1}^{1 / 2} \varphi, \mathrm{K}_{2}^{-1 / 2} \psi\right)=(\chi, \psi)$ for some $\chi$ in $D_{0}^{\perp}$, satisfying $\|\chi\| \leq a_{2}^{1 / 2}\|\varphi\|$. Since $\mathrm{K}_{1}^{1 / 2} \varphi$ is in $D_{0}^{\perp}$, we have $\chi=\mathrm{K}_{2}^{-1 / 2} \mathrm{~K}_{1}^{1 / 2} \varphi$ and hence $\left\|\mathrm{K}_{2}^{-1 / 2} \mathrm{~K}_{1}^{1 / 2} \varphi\right\| \leq a_{2}^{1 / 2}\|\varphi\|$ for any $\varphi$. Therefore

$$
\left\|\mathrm{K}_{2}^{-1 / 2} \psi\right\| \leq a_{2}^{1 / 2}|| \mathrm{K}_{1}^{-1 / 2} \psi \|
$$

for any $\psi \in \mathrm{K}_{1}^{1 / 2 \Omega}$. Similarly

$$
\left\|\mathrm{K}_{1}^{-1 / 2} \psi\right\| \leq a_{1}^{1 / 2}|| \mathrm{K}_{2}^{-1 / 2} \psi \|
$$

for any $\psi \in \mathrm{K}_{2}^{1 / 2} \mathcal{Q}$. Since the domain of $\mathrm{K}_{2}^{-1 / 2} \mathrm{~K}_{1}^{1 / 2}$ is $\mathcal{Q}$, the domain of $\mathrm{K}_{2}^{-1 / 2}$ contains $\mathrm{K}_{1}^{1 / 2} \mathcal{Q}$ which is the domain of $\mathrm{K}_{1}^{-1 / 2}$. Similarly the domain of $\mathrm{K}_{2}^{-1 / 2}$ contains the domain of $\mathrm{K}_{2}^{-1 / 2}$ and hence the two are equal. Thus $D^{+}$and its topology does not depend on the choice of $h$ and $\mathfrak{I}_{i}$.

Q.E.D.

Remark. In our preceding proof of the statement that $\mathrm{K}\left(\mathrm{I}_{i}\right)^{1 / 2} \varphi$ is in the domain of $\mathrm{K}^{-1 / 2}$, we have actually proved that for any bounded self-adjoint operators satisfying $A \geqq B \geqq 0$, the range of $A^{1 / 2}$ contains the range of $B^{1 / 2}$.

It is also possible to obtain $\bar{D}^{+}$by the following procedure. Introduce a family of seminorms $\|\psi \boldsymbol{\psi}\|_{\mathrm{I}} \equiv\left\|\mathrm{K}(\mathfrak{l})^{1 / 2} \psi\right\|$ to $D_{0}^{\perp}$ where $\mathfrak{l}$ runs over all elements in the Lie algebra. This defines a locally convex topology on $D_{0}^{\perp}$, which actually coincides with the topology of $D^{+}$. The completion of $D_{0}^{\perp}$ with respect to this topology gives $\bar{D}^{\leftarrow}$.

Lemma 6.3. Let $\mathfrak{S}$ be a Hilbert space, $G$ be a locally compact group, $Q(g)$ be a continuous unitary representation of $G$ on $\mathfrak{S}$, and $\phi(g)$ be a $\mathfrak{S}$-valued function on $G$ satisfying (4.24).

(1) If $\phi(g)$ is weakly measurable (i.e. $(\psi, \phi(g))$ is measurable for every $\psi$ ) and is locally essentially bounded (with respect to invariant measures on $G)$, then $\phi(g)$ is strongly continuous. 
(2) If $\|\phi(g)\|_{1}$ is measurable, then $\phi(g)$ is locally bounded.

(3) If $\phi(g)$ is weakly measurable and $\mathfrak{S}$ is separable, then $\|\phi(g)\|$ is measurable.

Proof. (1) First, we show that $\phi(g)$ is strongly continuous at $g=1$. By setting $g_{1}=1$ in (4.24), we have $\phi(1)=0$. Hence this amounts to proving $\lim _{g \rightarrow 1}\|\phi(g)\|=0$.

Let $\mathfrak{N}$ be a bounded neighbourhood of 1 such that $\|\phi(g)\| \leq R$ for almost all $g$ in $\mathfrak{R}$. Let $\mathfrak{R}_{1}$ and $\mathfrak{R}_{2}$ be neighbourhoods of 1 such that $\mathfrak{N}_{1} \mathfrak{N}_{2} \subset \mathfrak{N}$. Let $f$ be a continuous function with support in $\mathfrak{R}_{1}$ such that $\int f\left(g_{1}\right) \mathrm{d} g_{1}=1$ and $g \in \mathfrak{N}_{2}$. From (4.24), we have

$$
\begin{aligned}
(\psi, \phi(g))=\int[ & \left.f\left(g_{1} g^{-1}\right)-f\left(g_{1}\right)\right]\left(\psi, \phi\left(g_{1}\right)\right) \mathrm{d} g_{1} \\
& +\int\left([1-Q(g)] \psi, \phi\left(g_{1}\right)\right) \mathrm{d} g_{1} f\left(g_{1}\right),
\end{aligned}
$$

where $\mathrm{d} g_{1}$ is the right invariant measure on $G$. Hence

$$
|(\psi, \phi(g))| \leq R\|\psi\|\left|\int\right| f\left(g_{1} g^{-1}\right)-f\left(g_{1}\right)\left|\mathrm{d} g_{1}+\|\psi\|\left\|||\left[1-Q(g)^{*}\right] \phi\right\|,\right.
$$

where $\phi=\int \phi(g) f(g) \mathrm{d} g$, which converges weakly due to the measurability and essential boundedness of $\phi(g)$ on the bounded support of continuous $f$. This then implies $|(\psi, \phi(g))|\|\psi\|^{-1} \rightarrow 0$ as $g \rightarrow 1$ uniformly in $\psi$. Namely $i \phi(g) \| \rightarrow 0$.

From (4.24), we have $\left\|\phi\left(g_{1} g_{2}\right)-\phi\left(g_{2}\right)\right\|=\left\|\phi\left(g_{1}\right)\right\|$. Hence $\phi(g)$ is strongly continuous at any $g \in G$.

(3) This is obvious from $\| \phi(g)||^{2}=\sum_{j}\left|\left(\psi_{j}, \phi(g)\right)\right|^{2}$ for a countable orthonormal basis $\psi_{j}$.

(2) Let $\eta(g) \equiv\|\phi(g)\|$. It is measurable, $0 \leq \eta(g)<\infty$ and

$$
\eta\left(g_{1}\right)+\eta\left(g_{2}\right) \geq \eta\left(g_{1} g_{2}\right) \geq\left|\eta\left(g_{1}\right)-\eta\left(g_{2}\right)\right|
$$

due to (4.24). Let $\Delta$ be a bounded measurable subset of $G, v(\Delta)$ be a right invariant measure and

$$
\xi(\Delta, a)=v(\{g \in \Delta ; \eta(g)<a\}) / v(\Delta) .
$$

Since $g^{\prime} \in \Delta, \eta\left(g^{\prime}\right) \leq b$ implies $\eta\left(g^{\prime} g\right) \geq \eta(g)-b$ by (6.18), we have (for $b=\eta(g)-a$ )

$$
\xi(\Delta g, a) \leq 1-\xi(\Delta, \eta(g)-a)
$$


If $\mathfrak{N}$ is a sufficiently small neighbourhood of 1 , then the invariant measure of the complement of $\Delta g$ in $\Delta$ can be made smaller than given $\varepsilon>0$ for every $g \in \mathfrak{R}$. Then

$$
\xi(\Delta g, a) \geq \xi(\Delta, a)-\varepsilon / v(\Delta) .
$$

If $\eta(g)$ is locally unbounded at $g=1$, there exist $g_{j}, j=1,2, \cdots$ such that $g_{j} \in \mathfrak{N}$ and $\lim \eta\left(g_{j}\right)=+\infty$. By $\eta(g)<\infty$, we have $\lim _{b \rightarrow \infty} \xi(\Delta, b)=1$. Hence (6.20) and (6.21) imply, when $g_{j}$ is substituted into $g, \xi(\Delta, a)$ $-\varepsilon / v(\Delta) \leq 0$. This contradicts with $\lim _{a \rightarrow \infty} \xi(\Delta, a)=1$. Hence $\eta(g)$ is locally bounded at $g=1$.

From the first equation of (6.18), $\sup _{g \in \mathfrak{N}} \eta\left(g_{1} g\right) \leq \sup _{g \in \mathfrak{\Re}} \eta(g)+\eta\left(g_{1}\right)$. Hence $\eta(g)$ is locally bounded at any $g_{1} \in G$.

Q.E.D.

Lemma 6.4. If $\psi \in D^{+}$, then $\mathrm{K} \psi \in D^{-}$. The closure of this mapping is a unitary mapping from $\bar{D}^{+}$onto $D^{-}$. The sesquilinear form $(\psi, \phi)_{\mathfrak{L}}$ for $\psi \in D^{+}, \phi \in D^{-}$can be extended to $\psi \in \bar{D}^{+}$and it gives the duality between $D^{+}$and $D^{-}$. Here $(,)_{\mathbb{E}}$ is the inner product in $\mathrm{Q}$.

Proof. By definition, $\left\|\psi_{+}^{2}=\right\| \mathrm{K}^{1 / 2} \psi\left\|^{2}=\right\| \mathrm{K}^{-1 / 2} \mathrm{~K} \psi\left\|^{2}=\right\| \mathrm{K} \psi \|_{-}^{2}$ for any $\psi \in D^{+}\left(=D_{0}^{\perp}\right)$. Hence $\mathrm{K}$ is isometric and defined on a dense set $D^{+}$in $\bar{D}^{+}$. Further, $\mathrm{K} D^{+}$is dense in $D^{-}$. Hence the closure of $\mathrm{K}$ is a unitary mapping from $\bar{D}^{+}$onto $D^{-}$. For $\psi \in D^{+}, \phi \in D^{-}$, we have $(K \psi, \phi)_{-}=(\psi, \phi)_{\mathfrak{Q}}$. Since $\bar{D}^{+}$is self-dual with respect to $(,)_{+}$, $\mathrm{K} \bar{D}^{+}=D^{-}$is dual to $\bar{D}^{+}$with respect to $(,)_{\mathfrak{Q}}$. $\quad$ Q.E.D.

We note that for any operator $A$ which maps $D^{-}$into $D^{-}$, we can define $A^{*}$ as an operator on $\bar{D}^{+}$by $\left(A^{*} \psi, \phi\right)_{\mathfrak{L}}=(\psi, A \phi)_{\mathbb{R}}$ for $\psi \in \bar{D}^{+}, \phi \in D^{-}$. This definition coincide with $A^{*}$ in $\&$ if $\psi$ is in $D^{+}$.

Lemma 6.5. For each $\Psi \in \bar{D}^{+}$,

$$
\phi(g)=\left[Q(g)^{*}-1\right] \Psi
$$

is in $Z^{1}\left(G, D_{0}^{\perp}\right)$.

Proof. By (6.2), $Q(g)-1$ is a bounded mapping from $\mathfrak{Z}$ into $D^{-}$. The dual of $\mathbb{Q}$ and $D^{-}$with respect to $(,)_{\mathfrak{Z}}$ is $\mathbb{R}$ and $\bar{D}^{+}$. Hence $Q(g)^{*}-1$ can be considered as an adjoint of $Q(g)-1$ which must be a bounded mapping from $\bar{D}^{+}$into $\mathfrak{E}$, with the norm bounded by 
$d(g)$. Since $(Q(g)-1) D_{0}=0,\left(Q(g)^{*}-1\right) \bar{D}^{+} \perp D_{0} . \quad$ Further,

$$
\left(Q\left(g_{1}\right)-1\right) Q\left(g_{2}\right)=\left(Q\left(g_{1} g_{2}\right)-1\right)-\left(Q\left(g_{2}\right)-1\right)
$$

and hence (4.24) holds. By Lemma 6.2, we have, for any $\Psi \in D_{+}$ and $\Phi \in \mathbb{Q}$,

$$
\left(\left[Q(g)^{*}-1\right] \Psi, \Phi\right) \leq\|\Psi\|_{+}\|[Q(g)-1] \Phi\|_{-} \rightarrow 0
$$

as $g \rightarrow 1$. Therefore $\phi(g)$ is weakly continuous at $g=1$. Then $\phi(g)$ is weakly continuous at any $g$ by (4.24). We already know the local boundedness from $\|\phi(g)\| \leq d(g)\|\Psi\|_{+}$. (It also follows from the weak continuity.) By Lemma $6.3(1), \phi(g)$ is strongly continuous.

Q.E.D.

Let $H$ be a connected invariant subgroup of a Lie group $G$, $\hat{G}=G / H, H_{1}$ be the commutator group of $H, H_{2}$ be the maximal compact subgroup of the connected abelian group $H / H_{1}$ and $K \equiv$ $\left(H / H_{1}\right) / H_{2}$. Let $\tau(g), g \in G$ be defined by $\tau(g) h=g h g^{-1}$ for $h \in H$ and $\tau(g) k=[[\tau(g) h]]$ for $k=[[h]] \in K\left(h \in H,[h] \in H / H_{1}\right)$. Then for $h \in H, \tau(h)$ is the identity on $K$ and hence we can define $\tau(\hat{g})=\tau(g)$ on $K$ for $\hat{g}=g H \in \hat{G}$. Let $\gamma(\hat{g})$ be a measurable function $\hat{g} \in G / H$ with values in $G$ such that $\gamma(1)=1$ and $\hat{g}=\gamma(\hat{g}) H$. Let

$$
\alpha\left(\hat{g}_{1}, \hat{g}_{2}\right)=\left[\left[\gamma\left(\hat{g}_{1} \hat{g}_{2}\right)^{-1} \gamma\left(\hat{g}_{1}\right) \gamma\left(\hat{g}_{2}\right)\right]\right] .
$$

Let $k(g) \equiv\left[\left[\gamma(\hat{g})^{-1} g\right]\right]$ where $g \in G, \hat{g}=g H$. Then

$$
\alpha\left(\hat{g}_{1}, \hat{g}_{2}\right)=k\left(g_{1} g_{2}\right)\left\{\tau\left(g_{2}\right)^{-1} k\left(g_{1}\right)^{-1}\right\} k\left(g_{2}\right)^{-1}
$$

where $\hat{g}_{1}=g_{1} H, \hat{g}_{2}=g_{2} H$. From this, the following equality can easily be checked by using the commutativity of elements in $K$ :

$$
\alpha\left(\hat{g}_{2}, \hat{g}_{3}\right) \alpha\left(\hat{g}_{1} \hat{g}_{2}, \hat{g}_{3}\right)^{-1} \alpha\left(\hat{g}_{1}, \hat{g}_{2} \hat{g}_{3}\right)=\tau\left(\hat{g}_{3}\right)^{-1} \alpha\left(\hat{g}_{1}, \hat{g}_{2}\right) .
$$

Hence $\alpha$ determines a cohomology $\hat{\alpha}$ in $H^{2}(\hat{G}, K)$. Let $\alpha_{\gamma_{1}}$ and $\alpha_{\gamma_{2}}$ be the $\alpha$ corresponding to two choices $\gamma_{1}$ and $\gamma_{2}$ for $\gamma$. Then $\gamma_{1}(\hat{g}) \gamma_{2}(\hat{g})^{-1} \in H$ and hence $\delta(\hat{g})=\left[\left[\gamma_{1}(\hat{g})^{-1} \gamma_{2}(\hat{g})\right]\right]$ is a $K$-valued measurable function. We have

$$
\alpha_{\gamma_{1}}\left(\hat{g}_{1}, \hat{g}_{2}\right)=\alpha_{\gamma_{2}}\left(\hat{g}_{1}, \hat{g}_{2}\right)\left\{\delta\left(\hat{g}_{1} \hat{g}_{2}\right)\left(\tau\left(\hat{g}_{2}\right)^{-1} \delta\left(\hat{g}_{1}\right)\right)^{-1} \delta\left(\hat{g}_{2}\right)^{-1}\right\} .
$$

Hence $\hat{\alpha}$ docs not depend on the choice of $\gamma$ and is determined by $\hat{G}$. We write as $\hat{\alpha}(\hat{G})$. 
If $K_{1}$ is a $\tau(\hat{G})$ invariant connected subgroup of $K$ and $\hat{\alpha}(\hat{G})+K_{1}$ is a 0 cohomology in $K / K_{1}$, we shall call $K_{1}$ as an annihilator. In this case, there exists $K / K_{1}$ valued measurable function $\delta_{1}(\hat{g})$ on $\hat{G}$, of which $\left[\alpha_{\gamma_{1}}\left(\hat{g}_{1}, \hat{g}_{2}\right)\right]$ for a given $\gamma_{1}$ is the coboundary: $\left[\alpha_{\gamma_{1}}\left(\hat{g}_{1}, \hat{g}_{2}\right)\right]$ $=\delta_{1}\left(\hat{g}_{1} \hat{g}_{2}\right)\left[\tau\left(\hat{g}_{2}\right)^{-1} \delta_{1}\left(\hat{g}_{1}\right)^{-1}\right] \delta_{1}\left(g_{2}\right)^{-1}$. Then $\gamma_{2}(\hat{g})=\gamma_{1}(\hat{g}) \delta_{1}(\hat{g})$ satisfies $\left[\alpha_{\gamma_{2}}\left(\hat{g}_{1}, \hat{g}_{2}\right)\right]=0$.

Theorem 6.6. Let $G$ be a Lie group, $H$ be a connected invariant subgroup of $G, \hat{G}=G / H, \hat{g}$ be the coset containing $g \in G, H_{1}$ be the commutator subgroup of $G, H_{2}$ be the maximal compact subgroup of $H / H_{1},[h]$ be the coset containing $h \in H, K=\left(H / H_{1}\right) / H_{2},[[h]]$ be the coset containing $[h] \in H / H_{1}, \tau(\hat{g})$ be the representation of $\hat{G}$ on $K$ induced from the inner automorphisin of $G, \gamma(\hat{g})$ be a G-valued measurable function on $\hat{G}$ such that $\hat{g}=\gamma(\hat{g}) H$ and $\gamma(1)=1, \alpha_{\gamma}\left(\hat{g}_{1}, \hat{g}_{2}\right)$ $=\left[\left[\gamma\left(\hat{g}_{1} \hat{g}_{2}\right)^{-1} \gamma\left(\hat{g}_{1}\right) \gamma\left(\hat{g}_{2}\right)\right]\right], \quad \hat{\alpha}(\hat{G})$ be the cohomology class of $\alpha_{\gamma}$ in $H^{2}(\hat{G}, K)$, which is independent of $\gamma, K_{1}$ be an annihilator for $\hat{\alpha}(\hat{G})$, $[k]$ be the coset in $K / K_{1}$ containing $k \in K$, and $\gamma_{0}$ be a choice of $\gamma$ satisfying $\left[\alpha_{\gamma_{0}}\left(\hat{g}_{1}, \hat{g}_{2}\right)\right]=1$ for all $\hat{g}_{1}, \hat{g}_{2} \in \hat{G}$, which exists.

Let $\mathfrak{f}^{\prime}$ be the Lie algebra of the connected abelian group $K / K_{1}$, $\mu$ be a $\tau(\hat{G})$ invariant nonnegative hermitian form on $\mathfrak{f}^{\prime}+i \mathfrak{f}^{\prime}, \mathfrak{L}_{a}$ be the quotient of $\mathfrak{f}^{\prime}+i \mathfrak{f}^{\prime}$ by vectors of vanishing $\mu$-norm, and $\phi_{a}(g)=$ $\log \left[\left[\left[\gamma_{0}(\hat{g})^{-1} g\right]\right]\right]$.

Further let $Q_{b}(\hat{g})$ be a continuous unitary representation of $\hat{G}$ on a Hilbert space $\mathfrak{Q}_{b}$ and $\phi_{b}(\hat{g}) \in Z^{1}\left(\hat{G}, \mathfrak{Q}_{a} \oplus \mathfrak{Q}_{b}\right)$.

Let

$$
\begin{gathered}
\mathbb{Q}=\mathfrak{Q}_{a} \oplus \mathfrak{Q}_{b}, \quad Q(g)=\tau(\hat{g}) \oplus Q_{b}(\hat{g}), \\
\phi(g)=\phi_{a}(g)+\phi_{b}(\hat{g}) .
\end{gathered}
$$

Then $Q(g)$ is a continuous unitary representation of $G, Q(g)=1$ for $g \in H$, and $\phi(g) \in Z^{1}(G, \mathfrak{R})$.

Conversely, any such $Q(g)$ and $\phi(g)$ is of this form, i.e., the representation space can be decomposed as a direct sum of $Q(g)$ invariant subspaces $\mathfrak{R}_{a}$ and $\mathfrak{R}_{b}$, each of which is as described above.

Proof. Since $\bar{K} / K_{1}$ is connected, commutative and contains no compact part, the exponential mapping from $\mathfrak{f}^{\prime}$ onto $K / K_{1}$ is one to one and onto. Hence $\phi_{a}(g)$ is defined for all $g$. By the invariance of $\mu, \tau(\hat{g})$ is unitary. The equation (4.24) for $\mathfrak{Q}_{a}$ part follows from 
(6.25) and $\left[\alpha_{\gamma_{0}}\left(g_{1}, g_{2}\right)\right]=1 . \quad \tau(\hat{g})$ is continuous by construction. The continuity of $\phi$ follows from (6.28) and the measurability of $\phi$ due to Lemma 6.3.

We now prove the converse part. For $h \in H$, we have from $(4.24)$

$$
\phi(g)=\phi(g h)-\phi(h) .
$$

Further

$$
\begin{aligned}
Q(g)^{*} \phi(h) & =\phi(h g)-\phi(g) \\
& =\phi\left(g g^{-1} h g\right)-\phi(g)=\phi\left(g^{-1} h g\right)
\end{aligned}
$$

Let $\mathcal{Q}_{a}$ be the subspace spanned by $\phi(h), h \in H$. Then

$$
Q(g) \phi(h)=\phi\left(g h g^{-1}\right)=\phi(\tau(g) h)
$$

on this space. Hence it is invariant under $Q(G)$. Let $\mathfrak{Q}=\mathfrak{Z}_{a} \oplus \mathfrak{Q}_{b}$, $\phi(g)=\phi_{a}^{\prime}(g) \oplus \phi_{b}^{\prime}(g)$. Then $\phi_{b}^{\prime}(h)=0$ for $h \in H$. From (6.30), we have $\phi_{b}^{\prime}(g)=\phi_{b}^{\prime}(g h)$. Namely $\phi_{b}^{\prime}(g)$ depends only on $\hat{g}=g H$. Since $Q(g)$ commute with the projection on $\mathfrak{R}_{a}$ and $\mathfrak{Q}_{b}$, (4.24) holds for $\phi_{a}^{\prime}$ and $\phi_{b}^{\prime}$ separately. Hence we have the required property for $\mathfrak{Q}_{b}$ part. We now turn our attention to $\phi_{a}^{\prime}(g)$. From (6.30) we have

$$
\begin{aligned}
& \phi_{a}^{\prime}\left(h_{1} h_{2} h_{1}^{-1} h_{2}^{-1}\right)=0 \\
& \phi_{a}^{\prime}(\exp 2 \pi t \mathfrak{l})=t \phi_{a}^{\prime}(\exp 2 \pi \mathfrak{l})=0
\end{aligned}
$$

if $h_{i} \in H, e^{t \mathfrak{l}} \in H$, and $\exp 2 \pi \mathfrak{I}=1$. Hence $\phi_{a}^{\prime}(h)=0$ for $h \in H_{1}$ and $\phi_{a}^{\prime}(g)$ depends only on $g H_{1}$. Further $\phi_{a}^{\prime}(h)=0$ for $[h] \in H_{2}$ and hence $\phi_{a}^{\prime}(h)$ depends only on $[[h]] \in K$. From

$$
\phi_{a}^{\prime}(\exp t \mathfrak{l})=t \phi_{a}^{\prime}(\exp \mathfrak{l})
$$

$\phi_{a}^{\prime}(h)$ is real linear when considered as a function of $\log h$.

Let $K_{1}$ be the set of $k \in K$ for which $\phi_{a}^{\prime}(k)=0$. It is a connected subgroup of $K$ due to (6.30) and (6.35). Obviously $K_{1}$ must be $\tau(G)$ invariant. The Lie algebra $\mathfrak{k}_{1}^{\prime}$ of $K / K_{1}$ can be identified with a real linear subset of $\mathfrak{R}_{a}$, which spans $\mathfrak{R}_{a}$. The inner product of $\mathfrak{Q}_{a}$ induces an inner product of $\mathfrak{k}_{1}^{\prime}+i \mathfrak{k}_{1}^{\prime}$, which is $\tau(G)$ invariant, and positive semidefinite. Since $K$ is commutative, $\tau^{\prime} g$ ) defined on $K$ and hence on $K / K_{1}$ depends only on $\hat{g}=g H \in \hat{G}$. Hence we write it as $\tau(\hat{g})$. We may also write $\phi_{a}^{\prime}(h)=\log [[[h]]]$ for $h \in H$ according 
to the above identification.

Finally, for each $\hat{g} \in \hat{G}$, we fix $\gamma(\hat{g}) \in G$ such that $\hat{g}=\gamma(\hat{g}) H$. We shall compute

$$
\begin{aligned}
\log & {\left[\alpha\left(\hat{g}_{1}, \hat{g}_{2}\right)\right]=\phi_{a}^{\prime}\left(\gamma\left(\hat{g}_{1} \hat{g}_{2}\right)^{-1} \gamma\left(\hat{g}_{1}\right) \gamma\left(\hat{g}_{2}\right)\right) } \\
& =\phi_{a}^{\prime}\left(\gamma\left(\hat{g}_{1}\right) \gamma\left(\hat{g}_{2}\right)\right)+Q\left(\gamma\left(\hat{g}_{1}\right) \gamma\left(\hat{g}_{2}\right)\right)^{*} \phi_{a}^{\prime}\left(\gamma\left(\hat{g}_{1} \hat{g}_{2}\right)^{-1}\right) \\
& =\phi_{a}^{\prime}\left(\gamma\left(\hat{g}_{1}\right) \gamma\left(\hat{g}_{2}\right)\right)+Q\left(\gamma\left(\hat{g}_{1} \hat{g}_{2}\right)\right)^{*} \phi_{a}^{\prime}\left(\gamma\left(\hat{g}_{1} \hat{g}_{2}\right)^{-1}\right) \\
& =\phi_{a}^{\prime}\left(\gamma\left(\hat{g}_{1}\right) \gamma\left(\hat{g}_{2}\right)\right)-\phi_{a}^{\prime}\left(\gamma\left(\hat{g}_{1} \hat{g}_{2}\right)\right) \\
& =\phi_{a}^{\prime}\left(\gamma\left(\hat{g}_{2}\right)\right)+Q\left(\hat{g}_{2}\right)^{*} \phi_{a}^{\prime}\left(\gamma\left(\hat{g}_{1}\right)\right)-\phi_{a}^{\prime}\left(\gamma\left(\hat{g}_{1} \hat{g}_{2}\right)\right) .
\end{aligned}
$$

The orthogonal projection on $\mathfrak{f}^{\prime}$, in the real Hilbert space $\mathfrak{Q}_{a}$ with respect to the real part of the complex inner product of $\mathfrak{R}_{a}$, commutes with $Q(g), g \in G$. Applying it on (6.36) and using (6.31), we see that $\left[\alpha\left(\hat{g}_{1}, \hat{g}_{2}\right)\right]$ is cohomologous to 0 . Thus $K_{1}$ must be an annihilator.

We now define $\phi_{a}$ as in the theorem and $\phi_{b}(g)=\left(\phi_{a}^{\prime}(g)-\phi_{a}(g)\right)$ $\oplus \phi_{b}^{\prime}(g)$. We have $\phi_{b}(h)=0$ for $h \in H$.

Q.E.D.

We now study further structure of $\phi_{a}^{\prime}$. For this purpose, it is convenient to decompose at the start the representation $Q(g)$ of $G$ on the finite dimensional $\mathfrak{Q}_{a}$ into irreducible representations $Q_{j}(g)$ on $P_{j} \Omega_{a}$ where $P_{j}$ are projections. Then $P_{j} \phi_{a}^{\prime} \in Z^{1}\left(G, P_{j} \mathbb{R}_{a}\right)$ and we can discuss each $P_{j} \phi_{a}^{\prime}$. Equivalently, we shall assume that $Q(g), g \in G$ is already an irreducible representation on $\mathfrak{Q}_{a}$.

Let $H_{a 1}$ be the identity component of the subgroup $H_{a}$ consisting of all $g \in G$ such that $Q(g)=1$ on the subspace $\mathfrak{R}_{a}$. Let $H_{01}$ be the set of all $h \in H_{a_{1}}$ such that $\phi_{a}^{\prime}(h)=0$. Since $Q\left(g_{1} g g_{1}^{-1}\right)=Q\left(g_{1}\right) Q\left(g_{1}^{-1}\right)=1$ if $Q(g)=1, H_{a}$ and $H_{a 1}$ must be invariant subgroup of $G$. If $h \in H_{01}$, then

$$
\phi_{a}^{\prime}\left(g h g^{-1}\right)=Q(g) \phi_{a}^{\prime}(g h)+\phi_{a}^{\prime}\left(g^{-1}\right)=Q(g) \phi_{a}^{\prime}(g)+\phi_{a}^{\prime}\left(g^{-1}\right)=0 .
$$

Hence $g h g^{-1} \in H_{01}$ and $H_{01}$ is an invariant subgroup of $G$.

By the same reasoning as the proof of the previous theorem, $\hat{H}=H_{a 1} / H_{01}$ is faithfully represented by a real linear subset $\mathfrak{Z}_{a 1}$ of $\mathfrak{Z}_{a}$ ((6.30) and (6.35)) and hence it is abelian, does not contain any compact subgroup and is connected. $Q(g)$ on $\mathfrak{Q}_{a 1}$ coincides with the adjoint representation $\mathrm{Ad}(g)$ on $H_{a 1}$.

Let $P$ be the orthogonal projection on $\mathfrak{Q}_{a 1}$ in the real Hilbert space $\Omega_{a}$ with respect to the real part of the complex inner product of $\mathfrak{R}_{a}$. Let $\phi_{a}^{\prime \prime}(g)=P \phi_{a}^{\prime}(g)$. Since $\mathfrak{Q}_{a 1}$ is invariant under $Q(g)$, $\phi_{a}^{\prime \prime}$ 
belongs to $Z^{1}\left(G, \&_{a_{1}}\right)$. Let $H_{0}$ be the subgroup of $G$ consisting of all $g \in H_{a}$ such that $\phi_{a}^{\prime \prime}(g)=0$. By (6.37), it is an invariant subgroup. $H_{a} / H_{0}$ is faithfully represented by $\mathbb{Q}_{a 1}$ and is identifiable with $\hat{H}$. $G_{a}=G / H_{a}$ is faithfully represented by $g_{a}=g H_{a} \rightarrow \operatorname{Ad}(g)$ and $H_{a} / H_{0}$ is maximal abelian in $G / H_{0}$. Since $G_{a}$ is a connected Lie group with a faithful finite dimensional unitary representation, it is a direct product of a compact group and $R^{m}$.

For each $g \in G$, there exists an $h \in H_{a 1}$ such that $\phi_{a}^{\prime}(h)=\phi_{a}^{\prime \prime}(g)$ and $\xi(g) \equiv h H_{0} \in \hat{H}$ is uniquely determined. Let $\hat{H} \times G_{a}$ be the semidirect product with the multiplication law

$$
\left(h_{1}, g_{1}\right)\left(h_{2}, g_{2}\right)=\left(h_{1} \tau\left(g_{1}\right) h_{2}, g_{1} g_{2}\right)
$$

for $h_{1}, h_{2} \in \hat{H}, g_{1}, g_{2} \in G_{a}$. Then the mapping $g \rightarrow\left(\xi(g), g H_{a}\right)$ is a homomorphism onto $\hat{H} \times G_{a}$. If $g_{1} H_{a}=g_{2} H_{a}$, then $g_{1}^{-1} g_{2} \in H_{a}$ and $Q\left(g_{1}\right)=Q\left(g_{2}\right)$. If $\xi\left(g_{1}\right)=\xi\left(g_{2}\right)$ in addition, then $\phi_{a}^{\prime \prime}\left(g_{1}^{-1} g_{2}\right)=Q\left(g_{2}\right) * \phi_{a}^{\prime \prime}\left(g_{1}^{-1}\right)$ $+\phi_{a}^{\prime \prime}\left(g_{2}\right)=Q\left(g_{1}\right)^{*} \phi_{a}^{\prime \prime}\left(g_{1}^{-1}\right)+\phi_{a}^{\prime \prime}\left(g_{2}\right)=\phi_{a}^{\prime \prime}\left(g_{2}\right)-\phi_{a}^{\prime \prime}\left(g_{1}\right)=0$. Hence $g_{1}^{-1} g_{2} \in H_{0}$. Therefore $g H_{0} \in G / H_{0} \rightarrow\left(\xi(g), g H_{a}\right)$ is an isomorphism of $G / H_{0}$ onto $\hat{H} \times G_{a}$.

We now have the following structure: (1) a commutative group $\hat{H}$ isomorphic to $R^{n}$, (2) a real inner product $\mu$ on $\hat{H}$, (3) a connected subgroup $G_{a}$ of the orthogonal group on the real Hilbert space $L_{2}(H, \mu)$, and (4) an invariant subgroup $H_{0}$ of $G$ such that $G / H_{0}$ is isomorphic to the semidirect product $\hat{H} \times G_{a}$.

Conversely, suppose that such structure is given and $g H_{0} \rightarrow(\xi(g)$, $\eta(g))$ is the isomorphism of $G / H_{0}$ onto $\hat{H} \times G_{a}$. Then $Q(g)=\eta(g)$ is orthogonal matrix on $L_{2}(\hat{H}, \mu), \xi(g) \in Z^{1}\left(G, L_{2}(\hat{H}, \mu)\right)$ and

$$
\begin{aligned}
& H_{a}=\{g ; Q(g)=1, g \in G\}, \\
& H_{0}=\left\{g ; \xi(g)=0, g \in H_{a}\right\},
\end{aligned}
$$

$\hat{H}=H_{a} / H_{0}$ and $G_{a}=G / H_{a}$.

Finally we discuss $\phi_{a}^{\prime}(g)-\phi_{a}^{\prime \prime}(g)=\phi_{a}^{\prime \prime \prime}(g)$, which belongs to $Z^{1}(G$, $\left.\mathfrak{Q}_{a}\right)$. If $g \in H_{a 1}$, then $\phi_{a}^{\prime \prime \prime}(g)=0$, namely $\phi_{a}^{\prime \prime \prime}(g), g \in H_{a}$ depends only on $g H_{a 1} \in G_{a} / H_{a 1}$. We see from (6.31) and the continuity of $\phi_{a}^{\prime \prime \prime}(h)$ that $Q(g)=1$ for all $g \in G$ on $\phi_{a}^{\prime \prime \prime}(h), h \in H_{a} . \quad\left(H_{a} / H_{a 1}\right.$ is countable.) Since $Q$ is assumed to be an irreducible representation, either $\phi_{a}^{\prime \prime \prime}(h)$ $=0$ for all $h \in H_{a}$ or $Q(g)=1$ for all $g \in G$ and $H_{a}=H_{a 1}$. In either case we may assume $\phi_{a}^{\prime \prime \prime}(h)=0$ for $h \in H_{a}$. 
Now $Q(g)$ and $\phi_{a}^{\prime \prime \prime}(g)$ depends only on $g H_{a} \in G / H_{a}$ and $Q$ is a faithful unitary representation of $G / H_{a}=G_{a}$. If $G_{a}$ has no nontrivial connected invariant abelian subgroup, then $G_{a}$ is compact and hence $\phi_{a}^{\prime \prime \prime}$ is a coboundary by Theorem 7.1. Suppose that $G_{a}$ has an invariant abelian subgroup $G_{c}$ isomorphic to $R^{m}, m \neq 0$. The projection $E_{G_{c}}(0)$ on $Q\left(G_{c}\right)$ invariant vectors commutes with $Q(g), g \in G$. Since $Q$ is an irreducible and faithful representation of $G_{a}, E_{G_{c}}(0)$ $=0$. Since $\mathfrak{R}_{a}$ is of finite dimension, $\phi_{a}^{\prime \prime}(g)$ is coboundary due to Theorem 7.3.

Summarizing, we have

Theorem 6.7. (1) Let $G$ be a connected Lie group, $H_{a}$ and $H_{0}$ be invariant subgroups of $G$ such that (i) $H_{a} \supset H_{0}$ and $\hat{H} \equiv H_{a} / H_{0}$ is isomorphic to $R^{n}$, (ii) there exists an isomorphism $g \in G / H_{0} \rightarrow(\xi(g)$, $\eta(g)) \in \hat{H} \times G_{a}$, from $G$ onto the semidirect product of $\hat{H}$ with $G_{a} \equiv G / H_{a}$, where the adjoint representation of $G$ on $\hat{H}$ canonically induces the action of $G_{a}$ on $\hat{H}$, (iii) $G_{a}$ is a direct product of a compact group and $R^{m}$, (iv) $\hat{H}$ is maximal abelian in $G / H_{0}$, and $(v)$ there exists ad $(G)$ invariant inner product $\mu$ on $\hat{H}+i \hat{H}$. Then $\phi(g) \equiv \xi\left(g H_{0}\right) \in$ $L_{2}(\hat{H}+i \hat{H}, \mu) \equiv \mathfrak{Q}$ is in $Z^{1}(G, \mathcal{Q})$ relative to the unitary representation $Q(g)=\operatorname{ad}(g)$,

$$
\begin{aligned}
& H_{a}=\{g ; Q(g)=1, g \in G\}, \\
& H_{0}=\left\{g ; \phi(g)=0, g \in H_{a}\right\} .
\end{aligned}
$$

(2) Let $G$ be a connected Lie group, $Q(g)$ be an irreducible finite dimensional continuous unitary representation on $\mathbb{R}, \phi \in Z^{1}(G, \&)$. Let $H_{a}$ and $H_{0}$ be defined by (6.41) and (6.42). Then, $(a) H_{a}$ and $H_{0}$ are invariant subgroups satisfying (i)-(iv) of $(1),(b) \phi(h)$ depends only on $h H_{0} \in H_{a} / H_{0} \equiv \hat{H}$ and $\phi$ gives an isomorphism of $L_{2}(\hat{H}+i \hat{H}, \mu)$ onto $\&$ for an appropriate $\mu$, and $(c) \phi(g)-\xi\left(g H_{0}\right)$ is a coboundary.

(3) Let $G$ be a connected Lie group, $H$ be a connected invariant subgroup, $Q(g)$ be continuous unitary representation of $G$ on $\&$ without identity subrepresentation, $Q(g)=1$ for $g \in H, \phi \in Z^{1}(G, \&)$. Assume that $\&$ is spanned by $\phi(h), h \in H$. Let $H_{a}$ and $H_{0}$ be defined by (6.41) and (6.42). Then the conclusion (a), (b) and (c) of (2) hold.

In the above, $L_{2}(\hat{H}+i \hat{H}, \mu)$ denotes the quotient of $\hat{H}+i \hat{H}$ by the subspace of vectors with vanishing $\mu$ norm. 
The proof of (3) can be obtained by the following slight modification of the proof of (2). Since an identity subrepresentation is assumed to be absent, we have $H_{a}=H_{a 1}$. We have $G_{a}=G_{K} \times G_{c}$ where $G_{K}$ is compact, $G_{c}=R^{m}$ and $\times$ denotes the direct product. As before, $\left(1-E_{G_{c}}(0)\right) \phi_{a}^{\prime \prime \prime}$ is a coboundary due to Theorem 7.3. On $E_{G_{c}}(0) \phi_{a}^{\prime \prime \prime}, Q(g)=1$ for $g \in G_{c}$ and hence $Q(g)=1$ for all $g \in G$ on $E_{G_{c}}(0) \phi_{a}^{\prime \prime \prime}(h), h \in G_{c}$ by (6.31). By the absence of the identity subrepresentation, $E_{G_{c}}(0) \phi_{a}^{\prime \prime \prime}\left(G_{c}\right)=0$. Hence $E_{G_{c}}(0) \phi_{a}^{\prime \prime \prime}$ is a cycle for the compact group $G_{K}$ and must be a coboundary by Theorem 7.1.

Q.E.D.

\section{§. Determination of Cocycles}

Theorem 7.1. For a compact group $G, Z^{1}(G, \mathfrak{Q})=B^{1}(G, \mathfrak{Q})$.

Proof. Since $G$ is compact and $\phi(g)$ is continuous on $G, \phi(g)$ is uniformly bounded. We integrate (4.24) with respect to $g_{1}$ using the invariant measure on $G$ and obtain

$$
Q\left(g_{2}\right) * \int \phi\left(g_{1}\right) \mathrm{d} \mu\left(g_{1}\right)=\int \phi\left(g_{1} g_{2}\right) \mathrm{d} \mu\left(g_{1}\right)-\phi\left(g_{2}\right)
$$

If we set

$$
\Omega=\int \phi(g) \mathrm{d} \mu(g)
$$

and use the invariance of $\mu$, we obtain

$$
\phi\left(g_{2}\right)=\left(1-Q\left(g_{2}\right) *\right) \Omega
$$

where $\Omega$ is a vector independent of $g_{2}$.

Lemma 7.2. If $G$ is abelian and $Q(g), g \in G$ does not contain the identity representation, then $Z^{1}(G, \&)=B^{1}\left(G, \bar{D}^{+}\right)$.

Proof. The basic equation for our discussion is

$$
\left(Q\left(g_{1}\right) *-1\right) \phi\left(g_{2}\right)=\left(Q\left(g_{2}\right) *-1\right) \phi\left(g_{1}\right) .
$$

This equation is obtained as the difference of the equation (4.24) and the same equation with $g_{1}$ and $g_{2}$ interchanged, where the commutativity $g_{1} g_{2}=g_{2} g_{1}$ has been used.

Let $E_{\mathrm{I}_{1}}(\Delta)$ be the spectral projection for $Q\left(e^{t \mathrm{I}_{1}}\right)=\int e^{i t \lambda} E_{\mathrm{l}_{1}}(\mathrm{~d} \lambda)$ and $\mathrm{K}\left(\mathfrak{I}_{1}\right)$ be the operator introduced in $\S 6$. Then $\mathrm{K}\left(\mathfrak{I}_{1}\right)^{-1}$ is bounded for 
$E_{\mathfrak{l}_{1}}((-\infty, \infty)-(-\varepsilon, \varepsilon))$ for any $\varepsilon>0$. Hence

$$
\left[1-E_{\mathfrak{l}_{1}}((-\varepsilon, \varepsilon))\right] \phi(g)=\left(1-Q(g)^{*}\right) \Omega_{1},
$$

where

$$
\Omega_{1}=\mathrm{K}\left(-\mathfrak{l}_{1}\right)^{-1}\left[1-E_{\mathfrak{l}_{1}}((-\varepsilon, \varepsilon))\right] \int h(t) \phi\left(e^{t \mathfrak{I}_{1}}\right) \mathrm{d} t
$$

is a vector in the Hilbert space $\mathbb{Q}$ independent of $g$. Since $\mathfrak{I}_{1}$ is arbitrary, we see that

$$
(1-E(\Delta)) \phi(g)=\left(1-Q(g)^{*}\right) \Omega_{\Delta}
$$

for a vector $\Omega_{\Delta}$ in $(1-E(\Delta)) \mathcal{Q}$ where $E$ is the joint spectral projection for $Q\left(e^{t_{j} \mathfrak{l}_{j}}\right)$ and $\Delta$ is any neighbourhood of the origin.

We now define a functional $\Omega$ over a certain set of vectors in \& by

$$
(\Omega, \psi)=(\phi(g), \chi)
$$

if

$$
\psi=(1-Q(g)) \chi
$$

First we show that

$$
\psi=\left(1-Q\left(g_{1}\right)\right) \chi_{1}=\left(1-Q\left(g_{2}\right)\right) \chi_{2}
$$

implies

$$
\left(\phi\left(g_{1}\right), \chi_{1}\right)=\left(\phi\left(g_{2}\right), \chi_{2}\right)
$$

From (7.9) and (7.6) we have

$$
\begin{aligned}
& \left(\phi\left(g_{1}\right),[1-E(\Delta)] \chi_{1}\right)=\left(\Omega_{\Delta},\left(1-Q\left(g_{1}\right)\right) \chi_{1}\right)=\left(\Omega_{\Delta}, \psi\right) \\
& \quad=\left(\Omega_{\Delta},\left(1-Q\left(g_{2}\right)\right) \chi_{2}\right)=\left(\phi\left(g_{2}\right),[1-E(\Delta)] \chi_{2}\right) .
\end{aligned}
$$

By taking the limit of $\Delta$ shrinking to 0 and using the assumption $E(0)=0$, we obtain (7.10). Thus (7.7) does not depend on how $\psi$ is expressed in the form of eq. (7.8). Furthermore

$$
([1-E(\Delta)] \psi, \Omega)=\left(\psi, \Omega_{\Delta}\right), \psi \in \mathfrak{Q},
$$

for any neighbourhood $\Delta$ of the origin.

Next we show that $\Omega$ can be extended to be linear. Let

$$
\sum_{i=1}^{N}\left(1-Q\left(g_{i}\right)\right) \chi_{i}=0
$$


Then (7.6) implies

$$
\sum_{i=1}^{N}\left(\phi\left(g_{i}\right),[1-E(\Delta)] \chi_{i}\right)=0 .
$$

By taking the limit $\Delta \rightarrow 0$, we have again

$$
\sum_{i=1}^{N}\left(\phi\left(g_{i}\right), \chi_{i}\right)=0 \text {. }
$$

Hence $\Omega$ has a linear extension, which we denote again by $\Omega$.

Next, we use the fact that $\mathrm{K}\left(\mathrm{I}_{i}\right)^{-1 / 2}\left[1-Q\left(e^{t \mathrm{I}_{i}}\right)\right]$ is bounded and $\left[1-Q\left(e^{t \mathrm{I}_{i}}\right)\right]^{-1} \mathrm{~K}\left(\mathrm{I}_{i}\right)^{1 / 2} E(\Delta)$ is also bounded for a fixed $t$ if $\Delta$ is sufficiently small. If

$$
\psi=\left(1-Q\left(e^{t \Gamma_{i}}\right)\right) \chi,
$$

$\psi$ is in the domain of $\mathrm{K}\left(\mathfrak{I}_{i}\right)^{-1 / 2}$ and any vector $\psi$ in $E(\Delta)$ times the domain of $\mathrm{K}\left(\mathfrak{I}_{i}\right)^{-1 / 2}$ can be obtained by (7.14) with the following $\chi$ :

$$
\chi=\left[1-Q\left(e^{t \mathfrak{l}_{i}}\right)\right]^{-1} \mathrm{~K}\left(\mathfrak{I}_{i}\right)^{1 / 2}\left(\mathrm{~K}\left(\mathfrak{I}_{i}\right)^{-1 / 2} E(\Delta) \psi\right) .
$$

In particular, there is a constant $a_{i}$ such that

$$
\|E(\Delta) \chi\| \leq a_{i}\left\|\mathrm{~K}\left(\mathfrak{I}_{i}\right)^{-1 / 2} E(\Delta) \psi\right\| \text {. }
$$

Hence

$$
|(\Omega, E(\Delta) \psi)| \leq a_{i}|| \phi\left(e^{t \mathfrak{I}_{i}}\right)\|\| \mathrm{K}\left(\mathrm{I}_{i}\right)^{-1 / 2} E(\Delta) \psi \| .
$$

Let $\mathfrak{I}_{1} \ldots \mathfrak{I}_{n}$ span the Lie algebra. Since $G$ is commutative, $\mathrm{K}\left(\mathfrak{I}_{i}\right)$ commutes with each other.

If we split up $\Delta$ into mutually disjoint $n$ regions $\Delta_{i}, i=1, \cdots, n$ such that $\left(p_{1} \cdots p_{n}\right) \in \Delta_{i}$ implies $p_{i} \geq p_{j}$ for any $j$, then $E\left(\Delta_{i}\right)$ are mutually orthogonal projections with the sum $E(\Delta)$. We have

$$
\left\|\mathrm{K}^{-1 / 2} E\left(\Delta_{i}\right) \psi\right\|^{2} \geq(1 / n)\left\|\mathrm{K}\left(\mathfrak{I}_{i}\right)^{-1 / 2} E\left(\Delta_{i}\right) \psi\right\|^{2} .
$$

Therefore if $\psi$ is in the domain of $\mathrm{K}^{-1 / 2}$, then $E\left(\Delta_{i}\right) \psi$ is in the domain of $\mathrm{K}\left(\mathrm{Y}_{i}\right)^{-1 / 2}$. From (7.17) and (7.18), we have

$$
\left|\left(\Omega, E\left(\Delta_{i}\right) \psi\right)\right| \leq n^{1 / 2} a_{i}\left\|\phi\left(e^{t \mathfrak{I}_{i}}\right)\right\|\left\|\mathrm{K}^{-1 / 2} E\left(\Delta_{i}\right) \psi\right\| .
$$

Hence

$$
\begin{aligned}
& |(\Omega, E(\Delta) \psi)| \leq a\|E(\Delta) \psi\|_{-}, \\
& a=n \max _{i} a_{i}\left\|\phi\left(e^{t 1_{i}}\right)\right\| .
\end{aligned}
$$


We have the estimate (7.19) for any $\psi$ in $D^{-}$. Together with (7.11), we see that $\Omega$ is in the dual of $D^{-}$, namely

$$
\Omega \in \bar{D}^{+} .
$$

From (7.7) and (7.8), we have

$$
\phi(g)=\left(1-Q(g)^{*}\right) \Omega,
$$

which is the required result.

Q.E.D.

Theorem 7.3. Let $H$ be an invariant abelian subgroup of $G$ and $\phi \in Z^{1}(G, \mathfrak{R})$. Then

$$
\phi(g)=\phi_{1}(g)+\phi_{2}(g), \phi_{1}(g)=\left(1-Q(g)^{*}\right) \Omega
$$

where $\phi_{2}(g) \in Z^{1}\left(G, E_{H}(0) \mathcal{Q}\right), \Omega \in \bar{D}^{+}(H)$ and $\left(1-Q(g)^{*}\right) \Omega \in \mathcal{Q}$ for all $g \in G, E_{H}(0)$ is the projection on the subspace of vectors invariant under $H$ and $\bar{D}^{+}(H)$ is the $\bar{D}^{+}$for $H$.

Proof. Since $H$ is an invariant subgroup, $E_{H}(0)$ commutes with all $Q(g), g \in G$. Thus

$$
\begin{aligned}
& \phi_{1}(g)=\left(1-E_{H}(0)\right) \phi(g) \\
& \phi_{2}(g)=E_{H}(0) \phi(g)
\end{aligned}
$$

are both in $Z^{1}(G, \mathbb{R})$. By the previous lemma, $\phi_{1}(h), h \in H$ is of the form

$$
\phi_{1}(h)=\left(1-Q(h)^{*}\right) \Omega
$$

where $\Omega$ is in $\bar{D}^{+}(H)$. We shall show that

$$
\phi_{1}(g)=\left(1-Q(g)^{*}\right) \Omega .
$$

From the definition equation for a cocycle, we obtain

$$
\left(1-Q(h)^{*}\right) \phi_{1}(g)=\phi_{1}(h)-Q(g)^{*} \phi_{1}\left(g h g^{-1}\right) \text {. }
$$

If we put

$$
\begin{aligned}
\chi & \equiv(1-Q(h)) \psi-\left(1-Q\left(g h g^{-1}\right)\right) Q(g) \psi \\
& =(1-Q(g))(1-Q(h)) \psi \in D^{-}(H),
\end{aligned}
$$

we obtain, from (7.25) for $h \in H$ and $g h g^{-1} \in H$ and (7.27),

$$
(\chi, \Omega)=\left([1-Q(h)] \psi, \phi_{1}(g)\right) .
$$


Or somewhat differently written,

$$
\left([1-Q(g)] \chi_{1}, \Omega\right)=\left(\chi_{1}, \phi_{1}(g)\right)
$$

where $\chi_{1}=[1-Q(h)] \psi, \psi \in \mathbb{R}$.

If we make the simultaneous spectral decomposition of $Q(h)$, $h \in H$, then $K(H)$ is a multiplication of a function $\sum_{i}\left(1-\widetilde{h}\left(p_{i}\right)\right) \equiv \mathrm{K}(p)$. Since $Q(g) \mathrm{K}(\mathfrak{l}) Q(g)^{*}=\mathrm{K}(\operatorname{Ad}(g) \mathfrak{l}), \mathrm{K}(H)_{g} \equiv Q(g) * \mathrm{~K}(H) Q(g)$ is a multiplication of a function $\mathrm{K}(\operatorname{Ad}(g) p)$. Since $\operatorname{Ad}(g)$ is non singular and continuous in $g, K(H)^{1 / 2} K(H)_{g}^{-1 / 2}$ and $K(H)^{-1 / 2} K(H)_{g}{ }^{1 / 2}$ are locally bounded and hence uniformly bounded on a compact set. This implies that $Q(g)$ maps $D^{-}(H)$ into $D^{-}(H), D^{+}(H)$ into $D^{+}(H)$ and is uniformly bounded for $g$ in a compact set, with respect to the norms of $D^{-}(H)$ and $D^{+}(H)$, respectively.

Since the linear combinations of $[1-Q(h)] \psi, \psi \in \mathfrak{R}$ are dense in $D^{-}(H)$ as is seen from the simultaneous spectral decomposition of $Q(h), h \in H$, we see that (7.30) holds for all $\chi_{1} \in D^{-}(H)$ and

$$
\left(\chi_{1},\left(1-Q(g)^{*}\right) \Omega\right)=\left(\chi_{1}, \phi_{1}(g)\right) . \quad \text { Q.E.D. }
$$

Lemma 7.4. If $H$ is an invariant subgroup of $G$ and $Z^{1}(H, \mathfrak{Q})$ $=B^{1}(H, \mathfrak{R})$, then $\phi \in Z^{1}(G, \mathfrak{R})$ is always of the form

$$
\phi(g)=\left(1-Q(g)^{*}\right) \Omega+\phi_{2}(g),
$$

$\Omega \in\left(1-E_{H}(0)\right) \&, \phi_{2}(g) \in Z^{1}\left(G / H, E_{H}(0) \&\right)$ where $E_{H}(0)$ is the subspace of vectors invariant under $H$.

Proof. The proof is exactly the same as the previous one. In

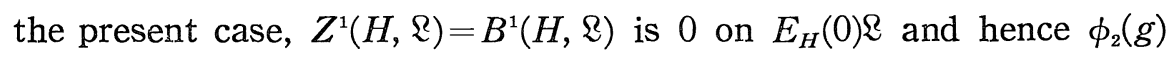
is in $Z^{1}\left(G / H, E_{H}(0) \mathfrak{R}\right)$.

Determination of cocycles. Assume $G$ is a connected Lie group. From the above theorems, we can analyze given $\phi(g), g \in G$ in the following way. Take maximal invariant abelian connected subgroup $G_{1}$ of $G$ and apply Theorem 7.3, Theorem 6.6 and Theorem 6.7. The problem is then reduced to $G / G_{1}=\hat{G}_{1}$. Continue this procedure until $\hat{G}$ has no invariant connected abelian subgroup. If the original $G$ is solvable, then $\hat{G}$ is trivial and the problem is completely solved. Otherwise we are left with a semisimple group. If it has invariant compact subgroup, we can apply Theorem 7.1 and Lemma 7.4 and 
proceed until $\hat{G}$ has no invariant compact subgroup.

We have not solved the problem for a semisimple Lie group.

Summarizing, we have the following.

Theorem 7.5. Let $G$ be a Lie group and $G_{j}, j=1, \cdots, n$ be an ascending sequence of invariant connected subgroups of $G$ such that $G_{1}=1$, each $G_{j} / G_{j-1}(j=2, \cdots, n)$ is abelian and $G / G_{n}$ is semisimple. Let $\mathbb{Q}$ be a Hilbert space, $Q$ be a continuous unitary representation of

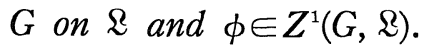

Then there exist mutually orthogonal invariant subspaces $\mathbb{Q}_{j}^{t}$, $j=1, \cdots, n$ and $\mathfrak{R}_{j}^{a}, j=1, \cdots, n$ of $\mathfrak{Q}$ such that

$$
\begin{aligned}
& \mathfrak{L}=\left(\bigoplus_{j=1}^{n} \mathfrak{Q}_{j}^{t}\right) \oplus\left(\bigoplus_{j=1}^{n} \mathfrak{Q}_{j}^{a}\right), \\
& Q(g)=\left(\oplus Q_{j}^{t}(g)\right) \oplus\left(\oplus Q_{j}^{a}(g)\right), \\
& \phi(g)=\left(\oplus \phi_{j}^{t}(g)\right) \oplus\left(\oplus \phi_{j}^{a}(g)\right), \\
& Q_{j}^{\prime}(g)=Q_{j-1}^{a}(g)=1 \text { for } \quad g \in G_{j}, j=2, \cdots, n, \\
& \phi_{j}^{t}(g)=\hat{\phi}_{j}^{t}\left(g G_{j}\right), \quad j=1, \cdots, n, \\
& \hat{\phi}_{j}^{t} \in B^{1}\left(G / G_{j}, \bar{D}^{+}\left(G_{j+1} / G_{j}\right)\right), \quad j=1, \cdots, n-1, \\
& \hat{\phi}_{n}^{t} \in Z^{1}\left(G / G_{n}, \mathfrak{R}_{n}\right), \\
& \phi_{j}^{a}(g)=0 \text { for } \quad g \in G_{j}, j=1, \cdots, n-1, \\
& Q_{n}^{a}(g)=1 \text { for } \quad g \in G .
\end{aligned}
$$

$L_{j}^{a}$ is finite dimensional and is spanned by $\phi_{j}^{a}(g), g \in G_{j+1}\left(G_{n+1}=G\right)$, $Q_{j}^{a}, j=1, \cdots, n-1$ has no identity subrepresentation. (The structure of $\phi_{j}^{a}$ is given by Theorem 6.7.)

If $G$ is solvable and $\mathfrak{R}$ has a finite dimension, then we can reformulate Theorem 7.5 as follows:

Corollary 7.6. Let $G$ be a solvable Lie group, \& be a Hilbert space of finite dimension, $Q$ be a continuous unitary representation of $G$ on $\&$ and $\phi \in Z^{1}(G, \mathfrak{R})$.

Then there exists an ascending sequence of invariant connected subgroups $G_{j}$ of $G$ and mutually orthogonal invariant subspaces $\hat{\mathfrak{L}}_{j}$ $(j=1, \cdots, n)$ such that $G_{1}=\{1\}, G_{n}=G, G_{j} / G_{j-1}$ is abelian $(j=2, \cdots, n)$, $\mathfrak{R}=\oplus \hat{\mathfrak{R}}_{j}, Q(g)=\oplus \hat{Q}_{j}(g), \phi(g)=\sum \hat{\phi}_{j}(g), \hat{Q}_{j}(g)=1$ for $g \in G_{j+1}, \hat{\phi}_{j}(g)=0$ for $g \in G_{i}, \hat{\phi}_{j}(g) \in \hat{\mathfrak{B}}_{j}(j=1, \cdots, n-1), \hat{\mathfrak{B}}_{j}$ is spanned by $\hat{\phi}_{j}(g), g \in G_{j+1}$ 
$(j=1, \cdots, n-1), \hat{\phi}_{n}(g)$ is a coboundary on $\mathbb{Q}$ and $\hat{Q}_{j}(g)$ is either an identity representation or without any identity subrepresentation $(j=1, \cdots, n-1)$. (The structure of $\hat{\phi}_{j}$ is given by Theorem 6.7.)

To obtain this form, we proceed in exactly the same manner as Theorem 7.5. All $\phi_{j}^{b}$ are coboundaries and all coboundaries are lumped together as $\hat{\phi}_{n}$ in the present Corollary. Each $Q_{j}^{a}$ in Theorem 7.5 can be split into a direct sum of an identity representation and a representation without any identity subrepresentation. Denoting $G_{j 0}=\left\{g \in G_{j}, \phi_{j}(g)=0\right\}, G_{j} / G_{j 0}$ is split into a direct sum of two invariant subgroups $G_{j A}$ and $G_{j B}$ of $G / G_{j 0}$, where $G_{A}$ consists of some central elements of $G / G_{j 0}$ and $G_{B}$ does not contain any central elements of $G / G_{j 0}$. If we inflate the ascending sequence $\left\{G_{j}\right\}$ of Theorem 7.5 by inserting the invariant subgroup $\left\{g ; g G_{0} \in G_{j A}\right\}$ between $G_{j-1}$ and $G_{j}$, we obtain the structure in Corollary 7.6 where $G_{j}$ and $n$ are different from those in Theorem 7.5.

\section{§. Determination of $\boldsymbol{c}(\boldsymbol{g})$}

When $\phi(g)$ is determined, $c(g)$ is to be determined from

$$
\begin{aligned}
c\left(g_{1} g_{2}\right)-c\left(g_{1}\right)-c\left(g_{2}\right) & =-\operatorname{Im}\left(\phi\left(g_{1} g_{2}\right), \phi\left(g_{2}\right)\right) \\
& =\operatorname{Im}\left(\phi\left(g_{1}\right), \phi\left(g_{2}^{-1}\right)\right)(\bmod 2 \pi) .
\end{aligned}
$$

Obviously, if $c_{0}(g)$ is one solution a general solution for $e^{i c(g)}$ is obtained by multiplying $e^{i c_{0}(g)}$ with an arbitrary unitary character of $G$. In the following, we omit $\bmod 2 \pi$. All equations involving $c(g)$ linearly are to be understood modulo $2 \pi$.

Lemma 8.1. If $Z^{2}(G, R)=B^{2}(G, R)$, then (8.1) has a solution.

Proof. Sufficient to prove that the righthand side of (8.1) belongs to $Z^{2}(G, R)$. Let $d\left(g_{1}, g_{2}\right)=\left(\phi\left(g_{1}\right), \phi\left(g_{2}^{-1}\right)\right)$. Then

$$
\begin{aligned}
d\left(g_{1},\right. & \left.g_{2} g_{3}\right)-d\left(g_{1} g_{2}, g_{3}\right) \\
= & \left\{\left(\phi\left(g_{1}\right), Q\left(g_{2}\right) \phi\left(g_{3}^{-1}\right)\right)+\left(\phi\left(g_{1}\right), \phi\left(g_{2}^{-1}\right)\right)\right\} \\
& -\left\{\left(Q\left(g_{2}\right)^{*} \phi\left(g_{1}\right), \phi\left(g_{3}^{-1}\right)\right)+\left(\phi\left(g_{2}\right), \phi\left(g_{3}^{-1}\right)\right)\right\} \\
= & d\left(g_{1}, g_{2}\right)-d\left(g_{2}, g_{3}\right) .
\end{aligned}
$$

This proves that $\operatorname{Im} d\left(g_{1}, g_{2}\right) \in Z^{2}(G, R)$.

For a simply connected semisimple group and a compact group, 
$Z^{2}(G, R)=B^{2}(G, R)$.

Theorem 8.2. If $\phi_{1} \in B^{1}(G, \mathbb{\&})$ and $\phi_{2} \in Z^{1}(G, \mathfrak{R})$, then both $\left(\phi_{1}\left(g_{1}\right)\right.$, $\left.\phi_{2}\left(g_{2}^{-1}\right)\right)$ and $\left(\phi_{2}\left(g_{1}\right), \phi_{1}\left(g_{2}^{-1}\right)\right)$ are coboundaries.

Proof. Let $\phi_{1}(g)=\left(1-Q(g)^{*}\right) \Omega, \Omega \in \Omega, c_{1}(g)=\left(\Omega, \phi_{2}\left(g^{-1}\right)\right), c_{2}(g)=$ $\left(\phi_{2}(g), \Omega\right)$. Then

$$
\begin{aligned}
& \left(\phi_{1}\left(g_{1}\right), \phi_{2}\left(g_{2}^{-1}\right)\right)=c_{1}\left(g_{1}\right)+c_{1}\left(g_{2}\right)-c_{1}\left(g_{1} g_{2}\right), \\
& \left(\phi_{2}\left(g_{1}\right), \phi_{1}\left(g_{2}^{-1}\right)\right)=c_{2}\left(g_{1}\right)+c_{2}\left(g_{2}\right)-c_{2}\left(g_{1} g_{2}\right) .
\end{aligned}
$$

To treat the case whore $\Omega$ is outside of $\Omega$, we need a few preparations.

Lemma 8.3. Let $g=e^{t !}, Q(g)=\int e^{i t \lambda} \mathrm{d} E(\lambda), E_{A} \equiv E([-A, A])$. Then

$$
\Omega_{A}(\mathfrak{l}) \equiv \lim _{t \rightarrow 0} t^{-1} E_{A} \phi(g)
$$

exists in $\mathfrak{Q}$ for any $\phi \in Z^{1}(G, \mathfrak{R})$. $\omega(\mathfrak{l}) \equiv E(0) \Omega_{A}(\mathfrak{l})$ is then independent of $A$ and

$$
\phi(g)=\left(1-Q(g)^{*}\right) \Omega+t \omega(\mathfrak{l})
$$

where $\Omega$ is in $\bar{D}_{+}$for one parameter group $\left\{e^{t !}\right\} .\left(1-E_{A}\right) \Phi \in \mathbb{Q}$ and $\left(-\mathrm{d} Q(\mathfrak{l}) E_{A}\right) * \Omega=\Omega_{A}(\mathfrak{l})-\omega(\mathfrak{l})$ where $\mathrm{d} Q(\mathfrak{l})=\left(\frac{d}{d t} Q\left(e^{t \mathfrak{l}}\right)\right.$ at $\left.t=0\right)$. If $f \in \mathscr{D}$ and $\int f(t) \mathrm{d} t=0$, then $Q(f) \Omega$ is in $\&$ where $Q(f)=\int Q(t) f(t) \mathrm{d} t$. Let $D_{G}$ be the set of $\int Q(g) \Psi f(g) \mathrm{d} g$ for $\Psi \in \mathcal{L}$ and $f$ in the class $\mathscr{D}$. For $\psi \in D_{G}$, the limit

$$
(\psi, \Omega(\mathfrak{l}))=\lim _{t \rightarrow 0}\left(\psi, t^{-1} \phi(g)\right)
$$

exists and satisfies

$$
\begin{aligned}
& \Omega\left(\lambda_{1} \mathfrak{I}_{1}+\lambda_{2} \mathfrak{I}_{2}\right)=\lambda_{1} \Omega\left(\mathfrak{I}_{1}\right)+\lambda_{2} \Omega\left(\mathfrak{I}_{2}\right) \\
& \mathrm{d} Q\left(\mathfrak{I}_{1}\right) \Omega\left(\mathfrak{I}_{2}\right)-\mathrm{d} Q\left(\mathfrak{I}_{2}\right) \Omega\left(\mathfrak{I}_{1}\right)=\Omega\left(\left[\mathfrak{I}_{1}, \mathfrak{I}_{2}\right]\right) .
\end{aligned}
$$

Proof. Since $Q(g) E_{A}, g=e^{t \downarrow}$ is holomorphic in $t$, it follows from (6.17) ( $\psi$ replaced by $E_{A} \psi$ ) that $\phi_{A}\left(e^{l !}\right)$ is strongly $\mathrm{C}^{\infty}$ in $t$, where $\phi_{A}(g)=E_{A} \phi(g)$. Since $\phi_{A}(1)=0$, we have $\left\|\phi_{A}\left(e^{t\urcorner}\right)\right\| \leq t a$ for $|t| \leq T$ and for a constant $a$.

Since $\phi \in Z^{1}(G, \mathfrak{R})$, we have 


$$
\phi\left(e^{t \mathfrak{l}}\right)=\sum_{j=0}^{n-1} Q\left(e^{-(j t / n) \mathfrak{r}}\right) \phi\left(e^{(t / n) \mathfrak{r}}\right) .
$$

Therefore, we have

$$
\begin{gathered}
\left\|\phi_{A}\left(e^{t \mathfrak{l}}\right)-n \phi_{A}\left(e^{(t / n) \mathfrak{l}}\right)\right\| \leq n c(t)\left\|\phi\left(e^{(t / n) \mathfrak{r}}\right)\right\|, \\
c(t)=\sup _{0 \leq s \leq t}\left\|\left(Q\left(e^{-s \mathfrak{l}}\right)-1\right) E_{A}\right\| .
\end{gathered}
$$

Hence

$$
\left\|\phi_{A}\left(e^{t \mathfrak{I}}\right)-n \phi_{A}\left(e^{(t / n) \mathfrak{r}}\right)\right\| \leq(1-c(t))^{-1} c(t)\left\|\phi_{A}\left(e^{t \mathfrak{}}\right)\right\| .
$$

Since $c(t)$ is of order $t$ for small $t$, we have for sufficiently small $t$ and a constant $b$,

$$
\left\|\phi_{A}\left(e^{t \mathrm{I}}\right)-\left(n^{-1}\right)^{-1} \phi_{A}\left(e^{t \mathrm{t} / n}\right)\right\|<b t^{2} .
$$

Hence

$$
\left\|(t / n)^{-1} \phi_{A}\left(e^{t l / n}\right)-(t / m)^{-1} \phi_{A}\left(e^{t l / m}\right)\right\|<2 b t .
$$

Further, we obtain from $\phi\left(g_{1}\right)-\phi\left(g_{2}\right)=Q\left(g_{2}\right)^{*} \phi\left(g_{1} g_{2}^{-1}\right)$

$$
\left\|\phi_{A}\left(e^{t \mathfrak{l} / n}\right)-\phi_{A}\left(e^{s \mathfrak{l}}\right)\right\| \leq\left\|\phi_{A}\left(e^{(t / n-s) \mathfrak{r}}\right)\right\|<a|t / n-s| .
$$

Let $t / n \geq s \geq t /(n+1)$. Then

$$
\left\|(t / n)^{-1} \phi_{A}\left(e^{t l / n}\right)-s^{-1} \phi_{A}\left(e^{s I}\right)\right\| \leq 2(n+1)^{-1} a .
$$

Hence by taking $t=\varepsilon$ and $s, s^{\prime}<\varepsilon^{2}$, we have

$$
\left\|s^{-1} \phi_{A}\left(e^{s I}\right)-s^{\prime-1} \phi_{A}\left(e^{s /}\right)\right\|<c \varepsilon
$$

for some constant $c$. Therefore $s^{-1} \phi_{A}\left(e^{s I}\right)$ is a Cauchy sequence and has a limit in $\mathbb{R}$.

If we denote the one parameter subgroup $\left\{e^{t l} ;-\infty<t<\infty\right\}$ tentatively by $H$, then $\phi \in Z^{1}(G, \mathfrak{l})$ implies $\phi \in Z^{1}(H, \mathfrak{l})$. Hence by Lemma 7.2, we have (8.6). From (8.6), $\omega(\mathfrak{l})=E(0) \Omega_{A}(\mathfrak{l})$ is independent of $A$.

Next by integrating with the weight $h(t)$, we have

$$
\int \phi\left(e^{t \mathfrak{l}}\right) h(t) \mathrm{d} t=\mathrm{K}(\mathfrak{l}) \Omega \in \mathfrak{Q} .
$$

Hence

$$
\left(1-E_{A}\right) \Omega=\mathrm{K}(\mathfrak{l})^{-1}\left(1-E_{A}\right) \int \phi\left({ }^{t \mathfrak{l}}\right) h(t) \mathrm{d} t \in \mathfrak{R} .
$$


Because $t^{-1}\left(1-Q\left(e^{t \mathfrak{l}}\right)\right) E_{A} \psi$ converges to $-\mathrm{d} Q(\mathfrak{l}) E_{A} \psi$ in $D^{-}(H)$, we also have $\left(-\mathrm{d} Q(\mathfrak{l}) E_{A}\right) * \Omega=\Omega_{A}(\mathfrak{l})-\omega(\mathfrak{l}) \in \mathbb{R}$ from (8.6). From these two conclusions, $Q(f) \Omega \in \mathbb{Z}$ for $\int f(t) \mathrm{d} t=0, f \in \mathscr{D}$ follows because $Q(f)\left(1-E_{A}\right)$ and $\mathrm{d} Q(\mathfrak{l})^{-1} Q(f) E_{A}$ are both bounded.

We now come to the second half of the lemma (which is incidentally not used in later discussions). It is known that $D_{G}$ is a common domain of any polynomial of infinitesimal generators of $Q(g)$. By splitting $\Omega$ in (8.6) into $\left(1-E_{A}\right) \Omega$ and $E_{A} \Omega$, we immediately have the existence of (8.7). At the same time,

$$
\phi\left(g_{1} g_{2}\right)=Q\left(g_{2}\right) * \phi\left(g_{1}\right)+\phi\left(g_{2}\right)
$$

with $g_{j}=\exp t \mathfrak{l}$, implies (8.8) in the first order in $t_{1}, t_{2}$, because $\phi(1)=0$. By using (8.21) repeatedly, we have

$$
\begin{aligned}
\phi\left(g_{1} g_{2} g_{1}^{-1}\right) & =Q\left(g_{1}\right) Q\left(g_{2}\right)^{*} \phi\left(g_{1}\right)+Q\left(g_{1}\right) \phi\left(g_{2}\right)+\phi\left(g_{1}^{-1}\right) \\
& =Q\left(g_{1}\right)\left\{\left(Q\left(g_{2}\right)^{*}-1\right) \phi\left(g_{1}\right)+\phi\left(g_{2}\right)\right\} .
\end{aligned}
$$

Hence

$$
\Omega\left(g_{1} \mathfrak{l}_{2}-\mathfrak{l}_{2}\right)=\left(Q\left(g_{1}\right)-1\right) \Omega\left(\mathfrak{l}_{2}\right)-Q\left(g_{1}\right) \mathrm{d} Q\left(\mathfrak{l}_{2}\right) \phi\left(g_{1}\right) .
$$

Taking the first order in $t_{1}$, we have (8.9).

Q.E.D.

We remark that it is possible to obtain (8.21) in a neighbourhood of the identity from (8.8) and (8.9) provided we have the convergences of several sequences.

Lemma 8.4. Let $\phi \in Z^{1}(G, \Omega)$. If $g$ is in the commutator subgroup of $G$, then $\phi(g) \in D^{-}(G)$.

Proof. We first note that $D^{ \pm}$is invariant under $Q(g)$ for any $g \in G$. The reason is as follows: If $\mathfrak{l}_{1} \cdots \mathfrak{l}_{n}$ is a basis of $\mathfrak{g}$, then $\operatorname{Ad}(g) \mathfrak{l}_{1} \cdots \operatorname{Ad}(g) \mathfrak{l}_{n}$ is also a basis of $g$ because $g$ has an inverse $g^{-1}$ and $\mathrm{Ad}(g)$ is nonsingular. Thus $\mathrm{K}$ and $Q(g) \mathrm{K} Q(g)^{-1} \equiv \mathrm{K}_{g}$ defines the same $D^{ \pm}$and the same topology by Lemma 6.2 , which establishes the invariance of $D^{ \pm}$under $Q(g)$.

Next we note the following consequences of (4.24):

$$
\begin{aligned}
& \phi\left(g_{1}^{-1} g_{2}^{-1} g_{1} g_{2}\right)=Q\left(g_{2}\right)^{*} \phi\left(g_{1}^{-1} g_{2}^{-1} g_{1}\right)+\phi\left(g_{2}\right) \\
& =Q\left(g_{2}\right) * Q\left(g_{1}\right)^{*} \phi\left(g_{1}^{-1} g_{2}^{-1}\right)+Q\left(g_{2}\right)^{*} \phi\left(g_{1}\right)+\phi\left(g_{2}\right) \\
& =Q\left(g_{2}\right) * Q\left(g_{1}\right) * Q\left(g_{2}\right)\left(\phi\left(g_{1}^{-1}\right)-\phi\left(g_{2}\right)\right)+Q\left(g_{2}\right)^{*} \phi\left(g_{1}\right)+\phi\left(g_{2}\right) \\
& =Q\left(g_{2}\right) *\left(1-Q\left(g_{1}^{-1} g_{2} g_{1}\right)\right) \phi\left(g_{1}\right)+\left(1-Q\left(g_{2}^{-1} g_{1}^{-1} g_{2}\right)\right) \phi\left(g_{2}\right)
\end{aligned}
$$


where we have used $Q(g)^{*} \phi\left(g^{-1}\right)=\phi(1)-\phi(g)=-\phi(g)$. Since $(1-$ $Q(g)) \mathfrak{L} \in D^{-}$for any $g$ and $D^{-}$is invariant under $Q(g)$, we see that $\phi(g) \in D^{-}$if $g=g_{1}^{-1} g_{2}^{-1} g_{1} g_{2}$. Further $\phi\left(g_{a} g_{b}\right)=Q\left(g_{b}\right) * \phi\left(g_{a}\right)+\phi\left(g_{b}\right)$ and hence $\phi(g) \in D^{-}$if $g$ is a product of elements of the form $g_{1}^{-1} g_{2}^{-1} g_{1} g_{2}$. Therefore $\phi(g) \in D^{-}$for all $g$ in the commutator subgroup of $G$.

Lemma 8.5. Let $G_{1}$ be the commutator subgroup of a connected Lie group $G$ and $G / G_{1}=K_{1} \cdot K_{2}$ where $K_{1}$ is compact and $K_{2}$ does not contain a compact subgroup. If $\phi \in Z^{1}(G, \&), g \in G,[g] \in K_{1}$ where $[g]$ denote the class of $g$ in $G / G_{1}$, then $\phi(g) \in D^{-}$.

Proof. Let Lie algebras for $G, G_{1}, K_{1}, K_{2}$ be $\mathfrak{g}, \mathfrak{g}_{1}, \mathfrak{f}_{1}, \mathfrak{k}_{2}$. Then $\mathrm{g} \bmod \mathfrak{g}_{1}=\mathfrak{f}_{1}+\mathfrak{l}_{2}$. Since $[g] \in K_{1}$, there exists $\mathfrak{l} \in \mathfrak{g}$, such that $[\mathfrak{l}] \in \mathfrak{f}_{1}$ and $[g]=\exp [\mathfrak{l}]$. Let $\exp t \mathfrak{l}=g(t)$. Then $[g(1)]=[g]$ and hence $g=g(1) g_{1}, g_{1} \in G_{1}$. By the previous lemma, $\phi\left(g_{1}\right) \in D^{-}$. Therefore, if $\phi(g(1)) \in D^{-}$, then $\phi(g)=Q\left(g_{1}\right)^{*} \phi(g(1))+\phi\left(g_{1}\right) \in D^{-}$. We now show that $\phi(g(1)) \in D^{-}$.

Let $E_{A}$ be defined as before in terms of the spectral projection of $Q(g(t))$. First consider the case where there exists a $t_{0}$ such that $\left[g\left(t_{0}\right)\right]=1$ and hence $g\left(t_{0}\right) \in G_{1}$. Then $\phi\left(g\left(t_{0}\right)\right) \in D^{-}$. Since $\left(1-E_{A}\right) \mathfrak{R}$ $\subset D^{-}$, we have $\Psi \equiv E_{A} \phi\left(g\left(t_{0}\right)\right) \in D^{-}$. If $A$ is sufficiently small, then from the known structure of $\phi(g)$ for an abelian group $\{g(t) ;-\infty$ $<t<+\infty\}$ (Lemma 7.2), we have

$$
E_{A} \phi(g(t))=\int_{-A-0}^{A+0} F(\lambda) \mathrm{d} E(\lambda) \Psi
$$

where $F(\lambda)$ is a function of class $\mathscr{D}$, coinciding with $\left(1-e^{-i t_{0} \lambda}\right)^{-1}(1-$ $\left.e^{-i t \lambda}\right)$ for $\lambda \in[-A, A], \lambda \neq 0$. Therefore

$$
E_{A} \phi(g(t))=\frac{1}{2 \pi} \int \widetilde{F}(s) \mathrm{d} s Q(g(-s)) \Psi .
$$

If we can show the continuity of $Q(g(-s)) \Psi$ in $D^{-}$, then (8.26) is in $D^{-}$and we have $\phi(g(t)) \in D^{-}$due to $\left(1-E_{A}\right) \mathfrak{L} \subset D^{-}$.

The continuity follows from the following inequality and Lemma 6.2 :

(8.27) $\left\|Q(g(-t)) \Psi-Q\left(g\left(-t^{\prime}\right)\right) \Psi\right\|_{-}=\| \mathrm{K}^{-1 / 2} Q(g(-t))\left(1-Q\left(g\left(t-t^{\prime}\right)\right) \Psi \|\right.$

$$
\begin{aligned}
& =\left\|\mathrm{K}_{t}^{-1 / 2}\left[1-Q\left(g\left(t-t^{\prime}\right)\right)\right] \Psi\right\| \\
& \leq a^{1 / 2}\left\|\mathrm{~K}^{-1 / 2}\left\{1-Q\left(g\left(t-t^{\prime}\right)\right)\right\} \Psi\right\| .
\end{aligned}
$$


Here $\mathrm{K}_{t}=Q(g(t)) \mathrm{K} Q(g(-t))$ and the local uniform bound $\| \mathrm{K}_{t}^{-1 / 2} \mathrm{~K}^{1 / 2} \mid$ $\leq a^{1 / 2}$ is obtained as follows. We take $\mathrm{K}_{1}=\mathrm{K}_{t}$ and $\mathrm{K}_{2}=\mathrm{K}$ in the proof of Lemma 6.2. Then $a_{1}$ in (6.13) is a locally bounded function of $t$.

We now consider the general case. If $\mathfrak{I}_{1} \cdots \mathfrak{I}_{m}$ are linearly independent basis of $\mathfrak{f}_{1}$ such that $\left\{e^{t t_{j}}\right\}$ for each $j$ is compact, then by repeated use of

$$
\phi\left(g e^{t l_{j}}\right)=Q\left(e^{t l_{j}}\right)^{*} \phi(g)+\phi\left(e^{t l_{j}}\right),
$$

and

$$
Q\left(g^{\prime}\right) D^{-} \subset D^{-},
$$

we have

$$
\phi\left(\exp \left(t_{1} \mathfrak{l}_{1}+\cdots+t_{m} \mathfrak{l}_{m}\right)\right) \in D^{-}
$$

for any $t_{1} \cdots t_{m}$.

Q.E.D.

Theorem 8.6. If $\phi \in B^{1}\left(G, \bar{D}^{+}\right)$, then $\operatorname{Im}\left(\phi\left(g_{1} g_{2}\right), \phi\left(g_{2}\right)\right) \in B^{2}(G, R)$, namely (8.1) has a solution.

Proof. Let $G, G_{1}, K_{1}, K_{2}$ be as in Lemma 8.5. Let $[g]$ be the class of $g$ in $G / G_{1}$ and $[[g]]$ be the element of $K_{2}$ such that $[[g]] \equiv[g] \bmod K_{1}$, and $[[g]]=\exp (t(g), \mathfrak{l})$, where $(t(g), \mathfrak{l})=\sum t_{i}(g) \mathfrak{l}_{i}$ and $\mathfrak{I}_{i}$ is a fixed basis of $\mathfrak{f}_{2}$. Then $t_{i}(g)$ is a representation of $G$ on the additive group $R$. Let $\hat{\mathfrak{l}}_{i}$ be an element of $\mathfrak{g}$ such that $\left(\hat{\mathfrak{l}}_{i} \bmod \mathrm{g}_{1}\right)=\mathfrak{l}_{i}$.

Next we note that if $g=g_{1} \cdots g_{n}$ and $\phi\left(g_{j}\right)-\phi_{j} \in D^{-}$, then $(\phi(g)-$ $\left.\sum_{j} \phi_{j}\right) \in D^{-}$. This is because

$$
\begin{aligned}
\phi(g)= & \sum_{j=1}^{n} \phi\left(g_{j}\right)+\left(Q\left(g_{n}\right)^{*}-1\right) \phi\left(g_{n-1}\right)+\left(Q\left(g_{n-1} g_{n}\right)^{*}-1\right) \phi\left(g_{n-2}\right) \\
& +\cdots+\left(Q\left(g_{2} g_{3} \cdots g_{n}\right) *-1\right) \phi\left(g_{1}\right)
\end{aligned}
$$

and $\left(Q(g)^{*}-1\right) \phi \in D^{-}$if $\phi \in \mathcal{Q}$.

Now $\phi(g)=\left(1-Q(g)^{*}\right) \Omega$ for $\Omega \in \bar{D}^{+}$. By Lemma 6.4, $\phi \in Z^{1}(G, \mathcal{L})$ and hence the previous lemmas are applicable. For $g \in G, g=$ $g^{\prime} e^{t_{1}(g) \mathfrak{r}_{1}} \ldots e^{\ell_{n}(g) \mathfrak{r}_{n}}$ for some $g^{\prime} \in G_{1} K_{1}$. We already know from Lemma 8.5 that $\phi\left(g^{\prime}\right) \in D^{-}$. Next $\phi\left(e^{t \mathfrak{l}_{2}}\right)-t \phi\left(e^{\mathfrak{I}_{2}}\right)=B \Omega, B=\left(t Q\left(e^{\mathfrak{I}_{i}}\right)^{*}-Q\left(e^{t \mathfrak{l}_{1}}\right) *\right.$ $+1-t)$. $\mathrm{K}\left(\mathrm{I}_{i}\right)^{1 / 2} \bar{D} \vdash \subset \mathcal{Q}$ and $\mathrm{K}\left(\mathrm{I}_{i}\right)^{-1 / 2} B \mathrm{~K}\left(\mathrm{I}_{i}\right)^{-1 / 2}$ is bounded, $B \Omega$ is in the domain of $\mathrm{K}\left(\mathrm{I}_{i}\right)^{-1 / 2}$ and hence $B \Omega \in D^{-}$. Thus we have 


$$
\phi(g)-\sum t_{i}(g) \phi\left(e^{\mathfrak{I}_{i}}\right) \in D^{-}
$$

Let

$$
c_{1}(g) \equiv\left(\Omega, \phi(g)-\sum t_{i}(g) \phi\left(e^{\mathfrak{l}_{i}}\right)\right) .
$$

Then, using $t\left(g_{1} g_{2}\right)=t\left(g_{1}\right)+t\left(g_{2}\right)$, we have

$$
\begin{aligned}
c_{1}\left(g_{1} g_{2}\right)-c_{1}\left(g_{1}\right)-c_{1}\left(g_{2}\right) \\
\quad=\left(\Omega, \phi\left(g_{1} g_{2}\right)-\phi\left(g_{1}\right)-\phi\left(g_{2}\right)\right) \\
\quad=\left(\Omega,\left(Q\left(g_{2}\right)^{*}-1\right) \phi\left(g_{1}\right)\right) \\
\quad=-\left(\phi\left(g_{2}^{-1}\right), \phi\left(g_{1}\right)\right)
\end{aligned}
$$

Hence $c(g)=\operatorname{Im} c_{1}(g)$ satisfies (8.1).

Q.E.D.

Since $\Omega$ in Theorem 7.3 is not necessarily in $\bar{D}^{+}(G)$, we can not in general apply Theorem 8.6 to $\phi_{j}^{t}, j=1, \cdots, n-1$ of Theorem 7.5. We also do not know the structure of a cocycle $\phi(g)$ for a semisimple group and hence we do not know whether $\operatorname{Im}\left(\phi_{n}^{t}\left(g_{1}\right)\right.$, $\left.\phi_{n}^{t}\left(g_{2}^{-1}\right)\right)$ is automatically a coboundary except when $G / G_{n}$ is simply connected in Theorem 7.5. We shall leave these problems for a future study and now consider the case where $\phi(g)=\sum_{j=1}^{n} \phi_{j}(g)$ in Corollary 7.6. We want a condition on $\phi_{j}$ such that there exists $c(g)(\bmod 2 \pi)$ satisfying

$$
c\left(g_{1} g_{2}\right)-c\left(g_{1}\right)-c\left(g_{2}\right)=\sum_{j=1}^{n-1} \operatorname{Im}\left(\phi_{j}\left(g_{1}\right), \phi_{j}\left(g_{2}^{-1}\right)\right) .
$$

In this case, the existence of $c$ is not automatic and we shall obtain an interesting structure.

We shall analyze (8.35) by an inductive procedure.

Theorem 8.7. Let $G_{(\alpha)}, G_{\alpha_{0}}, H_{\alpha}, H_{\alpha_{0}}$ be invariant subgroups of a connected Lie group $G^{(\alpha)}$ such that $G_{(\alpha)}$ is connected, $H_{a} \supset G_{(\alpha)}$, $H_{\alpha} \supset H_{\alpha_{0}}$ and $G_{\alpha_{0}}=H_{\alpha_{0}} \cap G_{(\alpha)}$. Let $Q_{\alpha}(g)$ be a continuous unitary representation of $G^{(\alpha)}$ on a finite dimensional space $\mathfrak{R}_{\alpha}$ and $\phi_{\infty} \in Z^{1}\left(G^{(\omega)}\right.$, $\left.\mathfrak{R}_{\infty}\right)$. Assume that $\phi_{\alpha}(g), g \in G_{(\infty)}$ spans $\mathfrak{L}_{\alpha}$,

$$
\begin{aligned}
& H_{a}=\left\{g ; Q_{\infty}(g)=1, g \in G^{\left(a^{a}\right)}\right\}, \\
& H_{\alpha_{0}}=\left\{g ; \phi_{a}(g)=0, g \in H_{\alpha}\right\},
\end{aligned}
$$

and $Q_{\infty}(g)$ is either an identity representation or without any identity subrepresentation. Assume that there exists a real valued measurable 
function $c_{a}(g)$ on $G^{(\infty)}$ satisfying

$$
\begin{aligned}
& c_{\alpha}\left(g_{1} g_{2}\right)-c_{\alpha}\left(g_{1}\right)-c_{\alpha}\left(g_{2}\right)=\operatorname{Im}\left(\phi_{\alpha}\left(g_{1}\right), \phi_{\alpha}\left(g_{2}^{-1}\right)\right) \\
& \quad+F_{\alpha}\left(g_{1} G_{(\omega)}, g_{2} G_{(\infty)}\right)
\end{aligned}
$$

modulo $2 \pi$ where $F_{\alpha}$ is a continuous function on $G^{(\alpha)} / G_{(\infty)} \times G^{(a)} / G_{(\infty)}$ satisfying

$$
F_{x}(1, x)=F_{x}(x, 1)=0, \quad x \in G^{(a)} / G_{(a)} .
$$

Then there exist subgroups $K_{\alpha}$ and $G_{\alpha}^{\prime}$ of $G^{(\alpha)}$ and $\Omega_{\alpha} \in \mathfrak{Q}_{\alpha}$ such that $G_{\alpha}^{\prime} \supset H_{\alpha 0}, G_{(\alpha)} \cap G_{\alpha}^{\prime}=G_{\alpha 0}, H_{\alpha}=G_{(\alpha)} G_{\alpha}^{\prime}, K_{\alpha} \cap H_{\alpha}=H_{\alpha 0}, G^{(\alpha)}=H_{\alpha} K_{\alpha}$, $G_{(\infty)} / G_{\alpha_{0}}$ and $G_{\alpha}^{\prime} / H_{\alpha_{0}}$ are isomorphic to $R^{n}$ and $R^{n^{\prime}}$ for some $n^{\prime} \leq n$, $K_{a} / H_{\alpha_{0}}$ is the direct product of a compact group and $R^{m}$,

$$
\phi_{\alpha_{0}}(g) \equiv \phi_{\alpha}(g)+\left(1-Q_{\alpha}(g)^{*}\right) \Omega_{\alpha}
$$

belongs to $Z^{1}\left(G^{(\infty)}, \mathfrak{\&}_{\infty}\right), \phi_{\omega_{0} 0}\left(K_{\infty}\right)=0, Q_{\infty}\left(g_{1}\right) \phi_{\alpha_{0}}\left(g_{2}\right)=\phi_{\alpha_{0}}\left(g_{1} g_{2} g_{1}^{-1}\right)$ and $\operatorname{Im}\left(\phi_{\alpha_{0}}\left(K_{\alpha} G_{\alpha}^{\prime}\right), \phi_{\alpha_{0} 0}\left(K_{\infty} G_{\infty}^{\prime}\right)\right)=0$.

Further, let

$$
c_{\infty 0}(g)=c_{\infty}(g)+(1 / 2)\left(\Omega_{\alpha},\left(Q_{\infty}(g)-Q_{\infty}(g)^{*}\right) \Omega_{\alpha}\right) .
$$

Then there exists a dual element $x_{\infty}$ of the Lie algebra $\mathfrak{g}_{(\infty)}$ of $G_{(\omega)}$ such that

$$
x_{\alpha}(\operatorname{Ad}(g) \mathfrak{l})=x_{\alpha}(\mathfrak{l})
$$

for $\mathfrak{l} \in \mathfrak{g}_{\alpha_{0} 0}, g \in G^{(\infty)}$ as well as for $\mathfrak{l} \in \mathfrak{g}_{(\infty)}, g \in K_{a}$ and

(8. 43) $\quad \operatorname{Im}\left(\phi_{\infty 0}\left(g_{1}\right), \phi_{\infty 0}\left(g_{2}\right)\right)=-(1 / 2) x_{a}\left(\log g_{1} g_{2} g_{1}^{-1} g_{2}^{-1}\right)$, where $g_{1} \in G_{(a)}, g_{2} \in H_{\alpha}$, log is taken modulo the commutator subgroup of $G_{\alpha 0}$.

The subgroup $G_{\alpha_{0}}$ must be connected and any $g \in G_{\alpha_{0}}$ can be written as $g=e^{\mathfrak{I}} g_{1}, \mathfrak{l} \in \mathfrak{g}_{\alpha_{0}}, g_{1}$ in the commutator subgroup of $G_{\infty \omega_{0}}$. For any such decomposition,

$$
c_{\infty}(g)=c_{\infty i 0}(g)=x_{\infty}(\mathfrak{l}) .
$$

$\exp i c_{\alpha_{0}}(g), g \in G_{\alpha_{0} 0}$ is a unitary character on $G_{\alpha_{0}}$.

Any $g$ in $G_{(\infty)}$ can be written as $g=e^{\mathfrak{l}} g_{1}, \mathfrak{l} \in \mathfrak{g}_{(\infty)}, g_{1} \in G_{\infty_{0}}$. For any such decomposition,

$$
c_{a}(g)=c_{a 0}(g)=x_{\alpha}(\mathfrak{l})+c_{\alpha}\left(g_{1}\right) .
$$


Any $g$ in $G^{(\alpha)}$ can be written as $g=g^{(3)} g^{(2)} g^{(1)}, g^{(1)} \in G_{(\alpha)}, g^{(2)} \in G_{\alpha}^{\prime}$, $g^{(3)} \in K_{a}$. For any such decomposition

$$
\begin{aligned}
c_{\alpha 0}(g)= & c_{\alpha_{0} 0}\left(g^{(3)} g^{(2)}\right)+c_{\alpha_{0}}\left(g^{(1)}\right) \\
& +(1 / 2) x_{\alpha}\left(\log g^{(2)} g^{(1)}\left\{g^{(2)}\right\}^{-1}\left\{g^{(1)}\right\}^{-1}\right),
\end{aligned}
$$

where the argument in the last term belongs to $G_{\alpha_{0}}$.

Let $c_{\alpha+1}$ be the restriction of $c_{\alpha 00}$ to $K_{\alpha} G_{\alpha}^{\prime} \equiv G^{(\alpha+1)}$. It satisfies $c_{a+1}(g)=c_{\omega_{0} 0}(g)$ for $g \in G_{\omega_{0} 0}$ and

$$
c_{a+1}\left(g_{1} g_{2}\right)-c_{\alpha+1}\left(g_{1}\right)-c_{\alpha+1}\left(g_{2}\right)=\hat{F}_{a t}\left(g_{1} G_{a 0}, g_{2} G_{\alpha_{0}}\right)
$$

where $\hat{F}_{a}\left(g_{1} G_{a_{0} 0}, g_{2} G_{a 0_{0}}\right) \equiv F_{\alpha}\left(g_{1} G_{(\alpha)}, g_{2} G_{(\alpha)}\right)$ for $g_{1}, g_{2}$ in $G^{(a+1)}$. $G^{(a+1)} / G_{\alpha_{0}}$ is isomorphic to $G^{(a)} / G_{(\alpha)}$. $G^{(a+1)}$ is connected.

Proof. From Theorem 6.7, there exists a measurable mapping $\xi$ from $g \in G^{(\alpha)}$ to $\xi(g) \in H_{\infty}$ such that $\xi(h)=h$ for $h \in H_{\infty}$ and $\xi\left(g_{1} g_{2}\right) H_{\infty 0}$ $=\left\{\xi\left(g_{1}\right) H_{\alpha_{0}}\right\}\left\{\tau\left(g_{1}\right) \xi\left(g_{2}\right) H_{\alpha_{0}}\right\}$. There also exists $\Omega_{\infty} \in \mathfrak{Q}_{\infty}$ such that

$$
\phi_{a}(g)=\phi_{a}(\xi(g))+\left(Q_{a}(g)^{*}-1\right) \Omega_{a} .
$$

Let $K_{a}$ be the set of $g$ such that $\xi(g) H_{\alpha_{0}}=H_{\alpha^{0} 0}$. It is a subgroup of $G^{(\infty)}$, isomorphic to $G^{(\infty)} / H_{a}, K_{\alpha} \supset H_{\alpha_{0}}, G^{(\infty)}=H_{\infty} K_{\infty}$ and $\phi_{\alpha_{0} 0}\left(K_{\infty}\right)=0$. $K_{\infty}$ is the direct product of a compact group and $R^{m}, H_{\alpha} / H_{\omega_{0}}$ is isomorphic to $R^{N}$ for some $N, G_{(\infty)} / G_{\alpha_{0}}$ is isomorphic to $R^{n}$ for $n \leq N \leq 2 n, \quad \phi_{\omega 0}(g)=\phi_{\alpha}(\xi(g))$ and $Q_{\alpha}\left(g_{1}\right) \phi_{\omega 0}\left(g_{2}\right)=\phi_{\alpha 0}\left(g_{1} g_{2} g_{1}^{-1}\right)$. Since $\phi_{\omega_{0}}(g)=\phi_{\alpha}(g)$ for $g \in H_{\alpha}, \phi_{\alpha_{0} 0}\left(G_{\alpha}\right)$ spans $\mathfrak{L}_{\alpha}$ and $K_{\alpha} \cap H_{\alpha_{0}}=H_{\alpha_{0} 0}$. Let $n^{\prime}=N-n$. We shall choose $G_{\alpha}^{\prime}$ after we have analyzed $c_{\alpha}(g)$.

Since $G_{(a)}$ is connected and $G_{(\omega)} / G_{\alpha 00}$ is simply connected, $G_{\alpha 0}$ is also connected.

We now consider (8.38). First, if $g_{1} \in G_{\alpha_{0} 0}$, then the right hand side vanishes and we have

$$
c_{a}\left(g g_{1}\right)=c_{\alpha}\left(g_{1} g\right)=c_{\alpha}(g)+c_{\omega}\left(g_{1}\right), g_{1} \in G_{\alpha 0}, g \in G^{(\infty)} .
$$

In particular, $\exp i c_{\sigma}(g), g \in G_{a 0}$, is a unitary character on $G_{a 0}$.

If $g_{1} \in G_{(\omega)}$, then

$$
\begin{aligned}
& c_{\alpha}\left(g_{1} g_{2}\right)-c_{\alpha}\left(g_{1}\right)-c_{\alpha}\left(g_{2}\right)=-\operatorname{Im}\left(\phi_{\alpha}\left(g_{1}\right), Q_{\alpha}\left(g_{2}\right) \phi_{\alpha}\left(g_{2}\right)\right), \\
& c_{\alpha}\left(g_{2} g_{1}\right)-c_{\alpha}\left(g_{1}\right)-c_{\alpha}\left(g_{2}\right)=-\operatorname{Im}\left(\phi_{\alpha}\left(g_{2}\right), \phi_{\alpha}\left(g_{1}\right)\right),
\end{aligned}
$$

where we have used $\phi_{\infty}\left(g^{-1}\right)=-Q_{\alpha}(g) \phi_{\infty}(g)$ and $Q_{\infty}\left(g_{1}\right)=1$. If we set $g_{2}=g_{1}^{-1}$ in (8.51) and use $c_{\omega}(1)=0$, we have 


$$
c_{\infty}\left(g_{1}^{-1}\right)=-c_{a}\left(g_{1}\right), g_{1} \in G_{(\infty)} .
$$

If $g_{2} \in H_{\alpha}$, then $Q_{\alpha}\left(g_{2}\right)=1$ and we have from (8.50) and (8.51)

$$
\frac{1}{2}\left\{c_{\alpha}\left(g_{1} g_{2}\right)+c_{\alpha}\left(g_{2} g_{1}\right)\right\}=c_{\alpha}\left(g_{1}\right)+c_{\omega}\left(g_{2}\right)
$$

$\bmod \pi$. Substituting $g_{1}^{-1}$ into $g_{1}$ and $g_{1} g_{2}$ and $g_{2} g_{1}$ into $g_{2}$ in (8.53), we have

$$
\begin{aligned}
& c_{\infty i}\left(g_{1} g_{2}\right)=c_{\omega}\left(g_{1}\right)+\frac{1}{2}\left\{c_{\omega_{i}}\left(g_{2}\right)+c_{\omega}\left(g_{1} g_{2} g_{1}^{-1}\right)\right\} \\
& c_{\omega i}\left(g_{2} g_{1}\right)=c_{\omega}\left(g_{1}\right)+\frac{1}{2}\left\{c_{\omega}\left(g_{1}^{-1} g_{2} g_{1}\right)+c_{\omega}\left(g_{2}\right)\right\}
\end{aligned}
$$

$\bmod \pi$ where we have used (8.52). We set

$$
\begin{aligned}
& k(g) g_{1} \equiv g_{1}\left(g g_{1} g^{-1}\right)^{-1} \\
& k^{\prime}(g) g_{1} \equiv\left(g^{-1} g_{1} g\right)^{-1} g_{1} .
\end{aligned}
$$

Since $Q_{a}\left(g_{2}\right)=1$ for $g_{2} \in H_{\alpha}$ and $\phi_{\infty}$ is faithful on $G_{(\infty)} / G_{\infty 00}, k(g) g_{1}$ and $k^{\prime}(g) g_{1}$ are in $G_{\alpha_{0}}$. Substituting $k\left(g_{2}\right) g_{1}$ and $k^{\prime}\left({ }_{2} g\right) g_{1}$ into $g_{1}$ and $g_{2}$ into $g$ in (8.49), we have

$$
\begin{aligned}
& c_{\infty}\left(g_{1} g_{2} g_{1}^{-1}\right)=c_{\omega}\left(g_{2}\right)+c_{\alpha}\left(k\left(g_{2}\right) g_{1}\right) \\
& c_{\omega}\left(g_{1}^{-1} g_{2} g_{1}\right)=c_{\omega}\left(g_{2}\right)+c_{\omega}\left(k^{\prime}\left(g_{2}\right) g_{1}\right)
\end{aligned}
$$

for $g_{1} \in G_{(\omega)}, g_{2} \in H_{\alpha}$. By substituting (8.58) and (8.59) into (8.54) and (8.55), we obtain

$$
\begin{aligned}
& c_{\infty}\left(g_{1} g_{2}\right)=c_{\infty}\left(g_{1}\right)+c_{\alpha}\left(g_{2}\right)+\frac{1}{2} c_{\alpha}\left(g_{1} g_{2} g_{1}^{-1} g_{2}^{-1}\right) \\
& c_{\omega}\left(g_{2} g_{1}\right)=c_{\infty}\left(g_{1}\right)+c_{\alpha}\left(g_{2}\right)+\frac{1}{2} c_{\omega}\left(g_{2}^{-1} g_{1}^{-1} g_{2} g_{1}\right)
\end{aligned}
$$

$\bmod \pi$ for $g_{1} \in G_{(\alpha)}, g_{2} \in H_{\alpha}$. If $g_{1} \in G_{\alpha_{0}}$ in (8.60), we obtain from (8.49),

$$
0=c_{o}\left(g_{1} g_{2} g_{1}^{-1} g_{2}^{-1}\right)=c_{\omega}\left(g_{1}\right)-c_{\alpha}\left(g_{2} g_{1} g_{2}^{-1}\right) .
$$

Thus we have restriction for $c_{\infty}$ :

$$
c_{\alpha}\left(g g_{1} g^{-1}\right)=c_{\alpha}\left(g_{1}\right), \quad g_{1} \in G_{a 0}, g \in H_{a} .
$$

From (8.50) and (8.60), we have 
(8. 64) $\quad \operatorname{Im}\left(\phi_{\alpha}\left(g_{1}\right), \phi_{\alpha}\left(g_{2}\right)\right)=-(1 / 2) c_{\alpha}\left(g_{1} g_{2} g_{1}^{-1} g_{2}^{-1}\right)$,

$$
g_{1} \in G_{(\omega)}, g_{2} \in H_{\infty}
$$

$\bmod \pi$. Since $Q_{a}(g)$ is unitary and $Q_{\alpha}(g) \phi_{\alpha}\left(g_{1}\right)=\phi_{\alpha}\left(g g_{1} g^{-1}\right)$ for $g_{1} \in H_{\alpha}$, we have

$$
c_{\alpha i}\left(g g_{1} g^{-1}\right)=c_{\alpha}\left(g_{1}\right),
$$

for $g \in G^{(\alpha)}, g_{1}=g_{2} g_{3} g_{2}^{-1} g_{3}^{-1}, g_{2} \in G_{(\alpha)}, g_{3} \in H_{\infty}$.

So far we have considered $c_{\alpha}$ and $\phi_{\alpha}$. Since $c_{\alpha}(g)=c_{\alpha 0}(g)$ and $\phi_{\alpha}(g)=\phi_{\infty 0}(g)$ for $g \in H_{\infty}$, all the results so far hold for $c_{\infty i 0}$ and $\phi_{\alpha_{0}}$ as well. We now consider $c_{\alpha 0}$ and $\phi_{\infty 0}$ for general $g \in G^{(a)}$.

If $g_{2} \in K_{\infty}$ in (8.48) and (8.51) where $c_{\alpha}$ and $\phi_{\infty}$ are replaced by $c_{\alpha_{0}}$ and $\phi_{\alpha_{0}}$, then the right hand sides are 0 . We substitute $g_{2}^{-1} g_{1} g_{2}$ into $g_{1}$ of (8.51) and subtract from (8.50). Then we obtain

$$
c_{\alpha}\left(g_{2}^{-1} g_{1} g_{2}\right)=c_{\alpha}\left(g_{1}\right), \quad g_{1} \in G_{(\alpha)}, g_{2} \in K_{\infty} .
$$

Since $G^{(a)}=H_{\alpha} K_{\alpha},(8.63)$ and (8.66) imply

$$
c_{\alpha}\left(g^{-1} g_{1} g\right)=c_{\alpha}\left(g_{1}\right), \quad g_{1} \in G_{\alpha_{0}}, \quad g \in G^{(\infty)} .
$$

Let

$$
y_{\alpha}(\mathfrak{l})=\exp i c_{\alpha}\left(e^{\mathfrak{l}}\right), \quad \mathfrak{l} \in \mathfrak{g}_{(\alpha)} .
$$

Since $c(g)$ is measurable and $\phi(g)$ is $C^{\infty}$, we see that $c(g) \bmod 2 \pi$ is $C^{\infty}$ by integrating (8.51) over $g_{2}$ with a class $\mathscr{D}$ function. Define

$$
x_{\infty}(\mathfrak{l})=\left.(\mathrm{d} / \mathrm{d} t) y_{\alpha}(t \mathfrak{l})\right|_{t=0}, \quad \mathfrak{l} \in \mathrm{g}_{(\alpha)} .
$$

From the definition,

$$
x_{\alpha}(t \mathfrak{l})=t x_{\alpha}(\mathfrak{l}), \quad \mathfrak{l} \in \mathrm{g}_{(\omega)} .
$$

Since $y_{a}(0)=1$, we have from $(8.60)$

$$
y_{\alpha}(\mathfrak{l})=\exp i x_{\omega}(\mathfrak{l}) .
$$

Hence

$$
c_{\alpha}\left(e^{\mathfrak{l}}\right)=x_{\alpha}(\mathfrak{l}) .
$$

Since $G_{(\omega)} / G_{\alpha 0}$ is commutative, $\left[\mathfrak{l}_{1}, \mathfrak{l}_{2}\right] \in \mathfrak{g}_{\alpha 0}$ for any $\mathfrak{l}_{1}, \mathfrak{l}_{2} \in \mathfrak{g}_{(\alpha)}$. Further

$$
x_{\alpha}\left(\left[\mathfrak{I}_{1}, \mathfrak{l}_{2}\right]\right)=0, \quad \mathfrak{l}_{1} \in \mathfrak{g}^{(\infty)}, \mathfrak{l}_{2} \in \mathfrak{g}_{\alpha_{0}}
$$


from (8.67). From (8.49), we have

$$
x_{\alpha}\left(\mathfrak{l}+\mathfrak{l}_{1}\right)=x_{\alpha}(\mathfrak{l})+x_{\alpha}\left(\mathfrak{l}_{1}\right), \quad \mathfrak{l} \in \mathfrak{g}_{(\infty)}, \mathfrak{l}_{1} \in \mathfrak{g}_{\alpha_{0} 0} .
$$

From (8.60), the Baker-Hausdorff formula, (8.74) and (8.73), we have

$$
x_{\alpha}\left(\mathfrak{l}_{1}+\mathfrak{l}_{2}\right)=x_{\alpha}\left(\mathfrak{l}_{1}\right)+x_{\alpha}\left(\mathfrak{l}_{2}\right), \quad \mathfrak{l}_{1}, \mathfrak{l}_{2} \in \mathfrak{g}_{(\alpha)} .
$$

From (8.70) and (8.75), we see that $x_{\alpha}$ is in the dual of $g_{(\alpha)}$.

(8.42) follows from (8.66) and (8.67). (8.44) follows from (8.49) and (8.72). (8.45) follows from (8.49). (8.46) follows from (8.60), (8.74) and (8.66) $\bmod \pi$. The argument in the last term of (8.46) is in $G_{\alpha_{0}}$ as long as $G_{\alpha}^{\prime} \subset H_{\alpha}$. (8.43) follows from (8.50) and (8.60) $\bmod \pi$. The two sides of (8.43) are continuous in $g_{1}$. Since it holds for $g_{1}=1$ and $G_{(\alpha)}$ is connected, (8.43) holds. Since the continuity of $c_{a}(\bmod 2 \pi)$ follows from $(8.38),(8.46)$ holds $\bmod 2 \pi$ by the same reason.

We now choose $G_{\alpha}^{\prime}$ so that $H_{\alpha} \supset G_{\alpha}^{\prime} \supset H_{\alpha_{0}}, H_{\alpha}=G_{(\alpha)} G_{\alpha}^{\prime}, G_{(\alpha)} \cap G_{\alpha}^{\prime}$ $=G_{\alpha 0}, G_{\alpha}^{\prime} / H_{\alpha_{0}}$ is isomorphic to $R^{n^{\prime}}$ and $\operatorname{Im}\left(\phi_{\alpha_{0}}\left(K_{\alpha} G_{\alpha}^{\prime}\right), \phi_{\alpha 0}\left(K_{\alpha} G_{\alpha}^{\prime}\right)\right)=0$. The last equation implies (8.47).

Let $\mathfrak{Q}_{\alpha}^{(1)}$ be the real Hilbert space consisting of elements in $\mathfrak{h}_{\alpha} / \mathfrak{h}_{\alpha 0}$ equipped with an inner product

$$
\mu_{\infty}\left(\hat{\mathfrak{I}}_{1}, \hat{\mathfrak{I}}_{2}\right)=\operatorname{Re}\left(\phi_{\omega 0}\left(e^{\mathfrak{I}_{1}}\right), \phi_{\alpha_{0} 0}\left(e^{\mathfrak{I}_{2}}\right)\right)
$$

where $\hat{\mathfrak{l}}_{j}=\mathfrak{l}_{j}+\mathfrak{h}_{\alpha_{0}} \in \mathfrak{h}_{\alpha} / \mathfrak{h}_{\alpha 0}, j=1,2$. Let

$$
\mu_{\alpha}\left(\hat{\mathfrak{I}}_{1}, \beta_{\omega} \hat{\mathfrak{I}}_{2}\right)=\operatorname{Im}\left(\phi_{\infty 0}\left(e^{\mathfrak{I}_{1}}\right), \phi_{\omega 0}\left(e^{\mathfrak{I}_{2}}\right)\right) \text {. }
$$

The real matrix $\beta_{a}$ on $\mathcal{R}_{\alpha}^{(1)}$ is antisymmetric and $\left\|\beta_{\alpha}\right\| \leq 1$. Let $\mathcal{R}_{\alpha}^{(2)}$ be the subspace $\left\{\mathfrak{I}+\mathfrak{h}_{\alpha_{0}} ; \mathfrak{l} \in \mathfrak{g}_{\left(\alpha_{0}\right)}\right\}$. We have $\operatorname{dim} \mathbb{R}_{\alpha}^{(2)}=n, \operatorname{dim} \mathbb{R}_{\alpha}^{(1)}=$ $n+n^{\prime}$.

Let $\beta_{\alpha}^{\prime}=E \beta_{\alpha} E$ for the projection $E$ on $\mathbb{2}_{\alpha}^{(2)}$. Since $\beta_{\alpha}^{\prime}$ is also real antisymmetric, there exists an orthonormal basis $z_{j}, j=1, \cdots, n$ in $\mathcal{R}_{\alpha}^{(2)}$ such that $\beta_{\alpha}^{\prime} z_{2 j-1}=a_{j} z_{2 j}, \beta_{\alpha}^{\prime} z_{2 j}=-a_{j} z_{2 j-1}, a_{j}>0$ for $j=1, \cdots, m$ and $\beta_{a}^{\prime} z_{j}=0$ for $j>2 m$ where $m$ is some integer not exceeding $n / 2$.

Let $\mathfrak{Q}_{a r}$ be the real linear subset of $\mathfrak{Q}_{\alpha}$ spanned by $\phi_{\alpha 0}\left(e^{\mathfrak{l}}\right), \mathfrak{l} \in z_{j}$, $j>2 m ; \phi_{a_{0} 0}\left(e^{\mathfrak{l}}\right), \mathfrak{l} \in z_{2 j}, j \leq m ;$ and $\phi_{\alpha_{0}}\left(e^{\mathfrak{l}}\right)-i a_{j} \phi_{\omega_{0}}\left(e^{\mathfrak{I}^{\prime}}\right), \mathfrak{l} \in z_{2 j-1}, \mathfrak{l}^{\prime} \in z_{2 j}$, $j \leq m$. Then the inner product of elements in $\mathbb{R}_{\alpha r}$ are all real. Since $\phi_{a 0}\left(G_{a}\right)$ span $\mathfrak{R}_{a}$ (as a complex linear set), $\mathfrak{R}_{a}$ must be the complexification of $\mathfrak{Q}_{a r}$. 
Let $\mathbb{Q}_{\alpha}^{(3)}$ be the orthogonal complement of $\mathbb{R}_{a}^{(2)}$ in $\mathbb{R}_{\alpha}^{(1)}$. For each $u \in \mathfrak{R}_{\alpha}^{(3)}$, let $s(u)$ be defined by

$$
s(u)=u+\sum_{j}^{\prime} a_{j}\left(1-\left(a_{j}\right)^{2}\right)^{-1}\left(z_{2 j}, \beta_{a} u\right) z_{2 j-1}
$$

where the summation is over $j \leq m$ such that $a_{j} \neq 1$. Let $s\left(\mathfrak{Q}_{\alpha}^{(3)}\right)=\mathfrak{Q}_{a}^{(4)}$.

By construction, $\mathbb{R}_{a}^{(2)}$ is a real linear subset of $\mathbb{R}_{\alpha}^{(1)}, \mathbb{R}_{\alpha}^{(3)} \cap \mathbb{R}_{\alpha}^{(2)}=\{0\}$, and $\mathbb{R}_{a}^{(3)}+\mathbb{R}_{a}^{(2)}=\mathbb{R}_{\alpha}^{(1)}$. Hence the same is true for $\mathbb{R}_{\alpha}^{(4)}$. Further, by construction of $\mathfrak{R}_{\alpha}^{(3)}$ and by (8.78), $\mathfrak{l} \in z \in \mathbb{R}_{\alpha}^{(4)}$ satisfies $\operatorname{Re}\left(\psi, \phi\left(e^{\mathfrak{l}}\right)\right)=0$ for all $\psi \in \mathbb{Q}_{\alpha r}$. Note that $\phi_{\alpha_{0}}\left(e^{\mathrm{l}}\right)-i a_{j} \phi_{\alpha_{0} 0}\left(e^{\mathrm{I}^{\prime}}\right)=0$ if $a_{j}=1$ because it has a 0 norm. This implies that $\phi\left(e^{\mathfrak{l}}\right) \in i \mathbb{R}_{\alpha r}$ for $\mathfrak{l} \in z \in \mathbb{R}_{\alpha}^{(4)}$ and hence $\operatorname{Im}\left(\phi\left(e^{\mathfrak{l}}\right), \phi\left(e^{\mathfrak{l}^{\prime}}\right)\right)=0$ for any $\mathfrak{l} \in \boldsymbol{z} \in \mathbb{R}_{\alpha}^{(4)}$ and $\mathfrak{I}^{\prime} \in \boldsymbol{z}^{\prime} \in \mathbb{R}_{\alpha}^{(4)}$.

Let $G_{\alpha}^{\prime}$ be the subgroup of $H_{\alpha}$ generated by $H_{\alpha_{0}}$ and $e^{\mathfrak{I}}, \mathfrak{I} \in z \in \mathbb{R}_{\alpha}^{(4)}$. $H_{\alpha} \supset G_{\alpha}^{\prime} \supset H_{\alpha 0}$ holds by construction. Since $\mathbb{R}_{\alpha}^{(3)}+\mathbb{Q}_{\alpha}^{(2)}=\mathbb{Q}_{\alpha}^{(1)}$ and $G_{\alpha}^{\prime} \supset$ $H_{\alpha 0}$, we have $H_{\alpha}=G_{(\alpha)} G_{\alpha}^{\prime}$. Since $\mathbb{R}_{\alpha}^{(4)} \cap \mathbb{R}_{\alpha}^{(2)}=\{0\}, G_{(\alpha)} \cap G_{\alpha}^{\prime}=G_{(\alpha)} \cap H_{\alpha 0}$ $=G_{\alpha_{0}} . \quad G_{\alpha}^{\prime} / H_{\alpha_{0}}$ is isomorphic to $\mathbb{R}_{\alpha}^{(4)} \sim R^{n^{\prime}}$. $\operatorname{Im}\left(\phi\left(g_{1}\right), \phi\left(g_{2}\right)\right)=0$ for $g_{1}, g_{2} \in G_{\alpha}^{\prime}$ by construction.

Since $G_{(\alpha)} \cap G^{(a+1)}=G_{\alpha_{0}}$ and $G^{(\alpha+1)} G_{(\alpha)}=G^{(\alpha)}, G^{(\alpha)} / G_{(\alpha)}$ is isomorphic to $G^{(a+1)} / G_{a 0}$. Since $G^{(\infty)}$ is connected, $G^{(\infty)} / G_{(\infty)}$ is connected. Since $G_{\omega_{0}}$ is connected in addition, $G^{(\infty+1)}$ is connected. Q.E.D.

By using this theorem, we can analyze a solution $c$ of (8.35) in the following manner. Let $G_{j}$ be as in Corollary 7.6, for a given connected solvable group $G$.

First we set $\alpha=2$ in Theorem 8.7 and consider $G^{(\omega)}=G, G_{(\omega)}=G_{2}$.

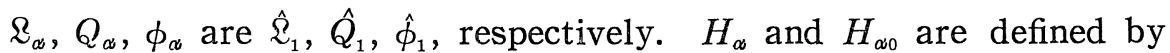
(8.36) and (8.37). $G_{\alpha_{0}}$ is defined as $H_{\alpha_{0}} \cap G_{(\omega)}$. The function $F_{a}$ is the $\operatorname{sum} \sum_{j=2}^{n-1} \operatorname{Im}\left(\hat{\phi}_{j}\left(g_{1}\right), \hat{\phi}_{j}\left(g_{2}^{-1}\right)\right)$. A solution $c$ of $(8.35)$ is taken as $c_{\alpha}$. Then Theorem 8.7 is applicable.

There exists an element $x_{\alpha}$ in the dual of $g_{(\alpha)}$ such that $c_{\alpha}\left(e^{\mathfrak{r}}\right)=$ $x_{\infty}(\mathfrak{l})$ for $\mathfrak{l} \in \mathfrak{g}_{(\alpha)}$. The group $G^{(\alpha+1)}$ contains $G_{\alpha_{0}}$ and $G^{(\alpha+1)} / G_{\alpha_{0}}$ is isomorphic to $G / G_{2}$. Both $G^{(\alpha+1)}$ and $G_{\alpha 0}$ are connected. $c_{\alpha}(g)$ for a general $g$ is given in terms of $x_{2}, \Omega_{2}$ and $c_{3}$ through formulas (8.40) and (8.46) where $\Omega_{2}$ is a vector in $\Omega_{1}$ and $c_{3}$ has to satisfy (8.47). In addition, $c_{3}\left(e^{\mathfrak{l}}\right)=x_{2}(\mathfrak{l})$ for $\mathfrak{l} \in \mathfrak{g}_{20}$. $\operatorname{Im}\left(\phi_{\alpha 00}\left(g_{1}\right), \phi_{\alpha 0}\left(g_{2}\right)\right)$ is fixed in terms of $x_{2}$ by (8.43). Since $G_{2}$ is abelian, this implies in particular that $\operatorname{Im}\left(\phi_{2}\left(g_{1}\right), \phi_{2}\left(g_{2}^{-1}\right)\right)=0$ for $g_{1}, g_{2} \in G_{2}$ (or else $c$ does not exist). (If $\phi_{\alpha_{0}}$ is given first, (8.43) is a restriction on $x_{2}$, for which 
a solution might not exist.)

Next we proceed to higher $c_{\alpha}$. We make an inductive assumption that we are given a connected subgroup $G^{(\infty)}$ of $G$, containing a connected invariant subgroup $G_{(\alpha-1) 0}=G^{(\alpha)} \cap G_{\alpha_{-1}}$ such that $G^{(\infty)} / G_{(\alpha-1) 0}$ is isomorphic to $G / G_{\alpha-1}$. We then look for $c_{\alpha}$ satisfying (8.38) with $\phi_{a}=\hat{\phi}_{a-1}$ and $F_{\alpha}=\sum_{j=\infty}^{n-1} \operatorname{Im}\left(\hat{\phi}_{j}\left(g_{1}\right), \hat{\phi}_{j}\left(g_{2}^{-1}\right)\right)$.

We apply Theorem 8.7 to the connected group $G^{(a)}$ and its invariant subgroup $G_{(\alpha)}=G^{(\alpha)} \cap G_{\alpha} . \quad G_{(\alpha)} / G_{(\alpha-1) 0}$ is isomorphic to $G_{\alpha} /$ $G_{a-1}$, which is connected. Hence $G_{(\alpha)}$ is also connected. $\mathfrak{Q}_{\alpha}, Q_{\alpha}, \phi_{\alpha}$ are taken to be $\hat{\mathfrak{Q}}_{\alpha_{-1}}, \hat{Q}_{\alpha_{-1}}, \hat{\phi}_{\omega_{-1}}$, respectively. $H_{\alpha}$ and $H_{\alpha_{0}}$ are defined by (8.36) and (8.37). $G_{\alpha 0}$ is defined as $H_{\alpha_{0}} \cap G_{(\alpha)}$. The function $F_{a}$ is as given above and we assume the existence of a solution $c_{a}$ of (8.38).

We then obtain a group $G^{(\alpha \mid 1)}$ such that it contains $G_{\alpha_{0}}=G^{(\alpha+1)}$ $\cap G_{\alpha}$ (which automatically contains $G_{(\alpha-1) 0}$ ) and $G^{(\alpha+1)} / G_{\alpha_{0}}$ is isomorphic to $G / G_{a}$. Both $G^{(a+1)}$ and $G_{\alpha 0}$ are connected. There exist an element $x_{a}$ in the dual of $g_{(a)}$ satisfying (8.42), an element $\Omega_{\alpha}$ in $\mathfrak{R}_{\alpha-1}$ and $c_{\alpha+1}$ satisfying (8.47) with $\hat{F}_{\alpha}=\sum_{j=\infty}^{n-1} \operatorname{Im}\left(\hat{\phi}_{j}\left(g_{1}\right), \hat{\phi}_{j}\left(g_{2}^{-1}\right)\right) . \quad c_{\alpha}(g)$ for a general $g$ is given in terms of $x_{\alpha}, \Omega_{\alpha}$ and $c_{\alpha+1}$ through formulas (8.40), (8.44), (8.45) and (8.46). $\operatorname{Im}\left(\phi_{\alpha 0}\left(g_{1}\right), \phi_{\alpha_{0}}\left(g_{2}\right)\right)$ is determined by (8.43) in terms of $x_{\alpha}$. $x_{\alpha}$ has to coincide with $x_{a-1}$ on $\mathrm{g}_{(\alpha-1) 0}$ and $c_{a+1}\left(e^{\mathfrak{l}}\right)$ has to coincide with $x_{a}(\mathfrak{l})$ for $\mathfrak{l} \in \mathfrak{g}_{\alpha_{0}}$.

Proceeding recursively, we obtain the following structure:

(1) Subgroups. $G^{(\alpha)}, \alpha=2, \cdots, n$ is a descending sequence of connected subgroups of $G$ and $K_{\alpha}, H_{\alpha}, G_{(\alpha)}, G_{\alpha}^{\prime}, G_{\alpha_{0}}, H_{\alpha_{0}}$ are subgroups of $G^{(\infty)}$. They are interrelated with each other and with $G_{j}$ of Corollary 7.6, by the relations : (i) $G_{(\infty)}=G^{\left(\omega^{\alpha}\right)} \cap G_{\alpha}, G_{\alpha_{0}}=G^{(\infty+1)} \cap G_{\alpha^{\prime}}$, $G_{(2)} \cdots G_{(\alpha)}=G_{\alpha}, G=G_{\omega^{t}-1} G^{(\alpha)}$. (ii) $G_{(\alpha-1) 0} g \rightarrow G_{\alpha-1} g$ gives isomorphisms of $G^{(\alpha)} / G_{(\alpha-1) 0}$ and $G_{(\alpha)} / G_{(\alpha-1) 0}$ onto $G / G_{\alpha-1}$ and $G_{\alpha} / G_{\alpha-1}$, respectively. (iii) $H_{\alpha} K_{\alpha}=G^{(\alpha)}, G_{\alpha}^{\prime} K_{\alpha}=G^{(\alpha+1)}, G_{(\alpha)} G_{\prime}^{\alpha}=H_{\alpha}, H_{\alpha} \cap G^{(\alpha+1)}=G_{\alpha}^{\prime}, H_{\alpha} \cap K_{\alpha}$ $=H_{\alpha 00}, G_{(\alpha)} \cap G_{\alpha}^{\prime}=G_{(\alpha)} \cap G^{(\alpha+1)}=G_{(\infty)} \cap K_{\alpha}=G_{(\alpha)} \cap H_{\alpha 0}=G_{\alpha 0}, \quad G_{x 0} \supset G_{(\alpha-1) 0}$ (an ascending sequence). (iv) $H_{\alpha}, G_{(\alpha)}, H_{\alpha 0}$, and $G_{\alpha 0}$ are invariant subgroups of $G^{(\alpha)}$. Besides $G^{(\alpha)}, G_{(\alpha)}$ and $G_{\alpha 0}$ are connected. $K_{\alpha} / H_{\alpha 0}$ is the direct product of a compact group and $R^{m_{a}}, H_{\alpha} / H_{\alpha_{0}}$ is isomorphic to $R^{N_{\alpha}}$ for some $N_{\alpha} \geq 0$, and is maximal abelian in $G^{(\alpha)} / H_{\alpha 0}$. (v) $G^{(2)}=G, G^{(n)}=G_{(n)}=H_{n}$ and $K_{n}=\{1\}$. 
(2) Hilbert spaces and cocycles. There exists a real linear mapping $p_{a}$ from the Lie algebra of $H_{\alpha}$ onto a total set in a complex Hilbert space $\mathfrak{L}_{a}\left(=\hat{\mathfrak{L}}_{\alpha-1}\right)$, equipped with an inner product $\hat{\mu}$. The kernel of $p_{\alpha}$ is $\mathfrak{S}_{\alpha_{0}}$. On $\mathfrak{R}_{\alpha}$, there exists a continuous unitary representation $Q_{a}(g)$ of $g \in G$ such that $Q_{a}(g)=1$ for $g \in G_{\alpha-1}$ and $Q_{\alpha}(g) p_{\alpha}=p_{\alpha} \operatorname{Ad}(g)$ for $g \in G^{(\alpha)}$ where $\operatorname{Ad}(g)$ is the adjoint representation of $G^{(\alpha)}$ on the Lie algebra of $H_{\alpha}$. For $g=g_{1} e^{\mathrm{I}} g_{3}, g_{1} \in G_{\omega-1}$, $\mathfrak{l} \in \mathfrak{h}_{\alpha}, g_{3} \in K_{\alpha}$,

$$
\phi_{\alpha}(g)=p_{\alpha} \mathfrak{l}+\left(Q_{\alpha}(g)^{*}-1\right) \Omega_{\alpha}
$$

is in $Z^{1}\left(G, \Omega_{a}\right)$ where $\Omega_{a} \in \mathfrak{Q}_{\alpha}$ is fixed.

$$
\mu_{\alpha}\left(\mathfrak{I}_{1}, \mathfrak{l}_{2}\right)=\operatorname{Re} \hat{\mu}_{\alpha}\left(p_{\infty} \mathfrak{I}_{1}, p_{\infty} \mathfrak{I}_{2}\right)
$$

is an $\operatorname{Ad}\left(G^{(\infty)}\right)$ invariant positive real inner product on $\mathfrak{h}_{\infty}$ with the kernel $\mathfrak{h}_{\alpha 0}$.

$$
\mu_{\alpha}\left(\mathfrak{I}_{1}, \beta_{\alpha} \mathfrak{I}_{2}\right)=\operatorname{Im} \hat{\mu}_{\alpha}\left(p_{\alpha} \mathfrak{I}_{1}, p_{2} \mathfrak{I}_{2}\right)
$$

defines an antisymmetric operator $\beta_{\alpha}$ on real Hilbert space $\mathfrak{h}_{\alpha} / \mathfrak{h}_{\alpha_{0}}$ such that

$$
\left\|\beta_{\alpha}\right\| \leq 1 .
$$

$\beta_{a}$ commutes with $\operatorname{Ad}(g), g \in G^{(a)}$.

(3) $x_{\infty}$ is in the dual of $g_{(\alpha)}$ and satisfies

$$
x_{\alpha}(\operatorname{Ad}(g) \mathfrak{l})=x_{\alpha}(\mathfrak{l})
$$

for $\mathfrak{l} \in \mathrm{g}_{\alpha 0}, g \in G^{(\alpha)}$ as well as for $\mathfrak{l} \in \mathrm{g}_{(\alpha)}, g \in K_{\alpha}$. Further,

$$
x_{\beta}\left|g_{\alpha_{0}}=x_{\alpha}\right| g_{\alpha_{0}} \quad(\beta>\alpha) .
$$

(4) $\beta_{\alpha}$ is related to $x_{\alpha}$ through

$$
\mu_{\alpha}\left(\mathfrak{l}_{1}, \beta_{\alpha} \mathfrak{I}_{2}\right)=-(1 / 2) x_{\alpha}\left(\left[\mathfrak{I}_{11}, \mathfrak{l}_{21}\right]+\left[\mathfrak{I}_{12}, \mathfrak{I}_{21}\right]+\left[\mathfrak{I}_{11}, \mathfrak{l}_{22}\right]\right)
$$

if $\mathfrak{l}_{j}=\mathfrak{l}_{j 1}+\mathfrak{l}_{j 2}, \mathfrak{l}_{j 1} \in \mathfrak{g}_{(\alpha)}, \mathfrak{l}_{j 2} \in \mathfrak{g}_{\alpha}^{\prime}, j=1,2$.

(5) $c_{\alpha}$ is given in terms of $x_{\alpha}, \Omega_{\alpha}$ and $c_{\alpha+1}$ by (8.40), (8.44), (8.45) and (8.46), where $c_{n+1}$ is to be taken 0. $\quad c \equiv c_{2}$ satisfies (8.35) with $\hat{\phi}_{j}=\phi_{j+1}$.

In the above analysis, we have shown that the pair $c$ and $\phi$ necessarily leads to the structures $(1) \sim(5)$. We have not listed all the restrictions, which could be extracted from our construction. 
(An example is a relation between adjoint representations of $G$ on Lie algebras of $G_{(\alpha)}$ and $H_{\alpha}$.) However, the structure and its property extracted above as $(1) \sim(5)$ are sufficient in the sense that, starting from the subgroup structure (1), the dual elements $x_{a}$ of (3) and real inner product $\mu_{a}$, we can always obtain (in a unique manner) a pair $\phi(g)$ and $c(g)$. More precisely, we have

Theorem 8.8. Let the structure of subgroups be given as in (1) above. Let $x_{\infty}$ be given as in (3) above. Let $\mu_{\infty}$ be an $\operatorname{Ad}\left(G^{(a)}\right)$ invariant positive real inner product on $\mathfrak{h}_{\alpha}$ with the kernel $\mathfrak{h}_{\alpha 0}$ such that $\beta_{a}$ defined by (8.85) satisfies (8.82). Then there exist $\mathfrak{\Omega}_{\alpha}, \Omega_{x}$, $Q_{a}, \phi_{\infty}$ and $c_{\alpha}$ which satisfy (2), (4) and (5) above. $\mathfrak{R}_{\alpha}, Q_{\alpha}, \phi_{a 0}$ and $c_{a 0}$ are unique while $\Omega_{\infty}$ is arbitrary.

Proof. Since (8.85) is antisymmetric in $\mathfrak{l}_{1}$ and $\mathfrak{l}_{2}$, the linear extension of

$$
\hat{\mu}_{\alpha}\left(\mathfrak{I}_{1}, \mathfrak{I}_{2}\right)=\mu_{\alpha}\left(\mathfrak{I}_{1}, \mathfrak{I}_{2}\right)+i \mu_{\infty}\left(\mathfrak{I}_{1}, \beta_{\infty} \mathfrak{I}_{2}\right)
$$

to the complexification $\mathfrak{h}_{\alpha}+i \mathfrak{h}_{\alpha}$ of $\mathfrak{h}_{\alpha}$, which is again denoted by $\hat{\mu}$, is a nonnegative inner product. The quotient of $\mathfrak{h}_{\alpha}+i \mathfrak{h}_{\alpha}$ by the kernel of $\hat{\mu}_{\infty}$ norm, considered as a complex Hilbert space with an inner product $\hat{\mu}_{\alpha}$, will be denoted by $\mathfrak{R}_{v}$. The natural homomorphism from real Hilbert space $\mathfrak{h}_{x}$ onto a total set in $\mathfrak{R}_{\infty}$ is denoted by $p_{a}$. The kernel of $p_{a}$ is $\mathfrak{h}_{x 0}$. (We do not necessarily demand that $p_{\infty} G_{(\infty)}$ be total in $\mathfrak{R}_{\infty}$.)

Due to (8.83) and $\operatorname{Ad}(g)\left[\mathfrak{l}_{1}, \mathfrak{l}_{2}\right]=\left[\operatorname{Ad}(g) \mathfrak{l}_{1},(g) \mathfrak{l}_{2}\right]$, we have $\mu_{w}\left(\operatorname{Ad}(g) \mathfrak{I}_{1}, \beta_{r} \operatorname{Ad}(g) \mathfrak{I}_{2}\right)=\mu_{w}\left(\mathfrak{I}_{1}, \beta_{w} \mathfrak{I}_{2}\right)$. Therefore,

$$
\hat{\mu}_{x}\left(\operatorname{Ad}(g) \mathfrak{l}_{1}, \operatorname{Ad}(g) \mathfrak{l}_{2}\right)=\hat{\mu}_{\alpha}\left(\mathfrak{I}_{1}, \mathfrak{l}_{2}\right), \quad g \in G^{(\alpha)} .
$$

This shows the extence of a unique continuous unitary representation $Q_{\alpha}(g)$ of $g \in G^{(\alpha)}$ such that $Q_{\alpha}(g) p_{\alpha}=p_{\alpha} \operatorname{Ad}(g) . Q_{\alpha}(g)=1$ if $g \in H_{\alpha}$.

Next we define $Q_{a}(g)=Q_{a}\left(g_{2}\right)$ if $g=g_{1} g_{2}, g_{1} \in G_{\alpha-1}, g_{2} \in G^{(\alpha)}$. Since $G=G_{x-1} G^{(\alpha)}$, any $g$ has such a decomposition. Since $G_{x-1} \cap G^{(\alpha)}$ $=G_{(\alpha-1)_{0}} \subset H_{\alpha}, Q_{w}\left(g_{2}\right)=Q_{\alpha}\left(g_{2}^{\prime}\right)$ for a different decomposition $g=g_{1}^{\prime} g_{2}^{\prime}$. Since $g \in G^{(a)} \rightarrow G_{\alpha} g$ is an isomorphism from $G^{(\alpha)} / G_{(v)}$ to $G / G_{a}$ and since $Q_{r}\left(g_{1} g_{2}\right)=Q_{r}\left(g_{2}\right)$ for $g_{1} \in G_{x}$, the extended $Q_{\infty}$ is a continuous unitary representation of $G$. 
For $g=g_{1} e^{\mathfrak{l}} g_{3}, g_{1} \in G_{a-1}, \mathfrak{l} \in \mathfrak{h}_{\alpha}, g_{3} \in K_{\alpha}$, we define

$$
\phi_{\infty 0}(g)=p_{\infty} \mathfrak{l} \text {. }
$$

Any $g \in G$ has such a decomposition, because $G=G_{\alpha-1} H_{\alpha} K_{\alpha}, H_{\alpha} / H_{\alpha 00}$ is exponential and $H_{\alpha 00} \subset K_{\alpha}$. Since $G_{\alpha-1} \cap H_{\alpha} K_{\alpha}=G_{(\alpha-1) 0} \subset G_{\alpha 0}, H_{\alpha} \cap$ $K_{\alpha}=H_{a 0}$, and $p_{a} \mathfrak{l}=p_{\infty} \mathfrak{l}^{\prime}$ for $e^{\mathfrak{l}}=g_{1} e^{\mathfrak{I}^{\prime}} g_{3}, g_{1} \in G_{\alpha 00}, g_{3} \in H_{a 00}$, the above definition of $\phi_{\alpha 0}(g)$ is independent of the decomposition of $g$. (Note the following: For $\mathfrak{l}_{1} \in \mathfrak{g}_{\alpha 00}, \mathfrak{l}^{\prime} \in \mathfrak{h}_{\alpha}, \operatorname{Ad}\left(e^{\mathfrak{l}_{1}}\right) \mathfrak{l}^{\prime}-\mathfrak{l}^{\prime}=\sum_{n=1}^{\infty}(n !)^{-1} \operatorname{Ad}\left(\mathfrak{l}_{1}\right)^{n} \mathfrak{l}^{\prime} \in$ $\mathfrak{h}_{x 0}$. Since $G_{x 0}$ is connected, $\mathfrak{l}^{\prime \prime}=\operatorname{Ad}\left(g_{1}\right) \mathfrak{l}^{\prime}$ satisfies $p_{a} \mathfrak{l}^{\prime \prime}=p_{\alpha} \mathfrak{l}^{\prime}$ for any $g_{1} \in G_{\alpha 0}$. Next, if $e^{\mathfrak{l}}=e^{\mathfrak{l}^{\prime \prime}} g_{3}^{\prime}$ for $g_{3}^{\prime}=g_{1} g_{3} \in H_{\alpha 0}, \mathfrak{l}, \mathfrak{l}^{\prime \prime} \in \mathfrak{h}_{\alpha}$, then $\mathfrak{l}-\mathfrak{l}^{\prime \prime} \in \mathfrak{h}_{\infty 0}$ because $H_{\alpha} / H_{\alpha 00}$ is isomorphic to $R^{N_{a}}$. Hence $p_{a} l^{\prime \prime}=p_{\alpha}{ }^{\prime}$.)

We now prove that $\phi_{\infty 0} \in Z^{1}\left(G, \mathfrak{R}_{\infty}\right)$. We already know from earlier results that $\phi_{x \neq 0}$ restricted to $G^{(\alpha)}$ is in $Z^{1}\left(G^{(\alpha)}, \mathbb{R}_{\alpha}\right)$. We also know that, for $g \in G_{\omega-1}$ and $g_{1} \in G^{(\alpha)}, Q_{\alpha}(g)=1, \phi_{\infty}(g)=0, \phi_{\infty}\left(g g_{1}\right)=\phi_{\alpha}\left(g_{1}\right)$ and $\phi_{\alpha}\left(g_{1} g\right)=\phi_{\alpha}\left(g_{1} g g_{1}^{-1} g_{1}\right)=\phi_{\alpha}\left(g_{1}\right)$. Here the last equality is due to $g_{1} g g_{1}^{-1} \in G_{a-1}$ if $g \in G_{\alpha^{\alpha-1}}$. Therefore $\phi_{a 0} \in Z^{1}\left(G, \mathfrak{R}_{a}\right)$.

We now define $c_{a}$ assuming that $c_{a+1}$ is already defined. To start the inductive procedure, we take $c_{n+1} \equiv 0$.

The quotient of $G_{\alpha 0}$ by its commutator subgroup, say $G_{\alpha 0}^{\prime}$, is connected and abelian. Hence it is exponential and any $g \in G_{\alpha 0}$ can be written as $g=e^{\mathfrak{l}} g_{1}, \mathfrak{l} \in \mathfrak{g}_{r 00}, g_{1} \in G_{\alpha 0}^{\prime}$. Hence (8.44) defines $c_{\infty 00}(g)$ for all $g \in G_{a 00}$. Suppose $e^{\mathfrak{l}} e^{-\mathfrak{l}^{\prime}}$ is in the commutator subgroup. Since $G_{x x} / G_{x 0}^{\prime}$ is exponential, $\mathfrak{l}-\mathfrak{l}^{\prime} \in \mathrm{g}_{\alpha 0}^{\prime}$. From special case of (3.83) with $g \in G_{x 00}$, we have $x_{x}\left(g_{\alpha 0}^{\prime}\right)=0$. Hence the definition (8.44) is independent of the decomposition $g=e^{\mathrm{I}} g_{1}$.

If $g=e^{\mathfrak{l}} g_{1}, g^{\prime}=e^{\mathfrak{I}^{\prime}} g_{1}^{\prime}, \mathfrak{l}, \mathfrak{l}^{\prime} \in \mathrm{g}_{x_{0}}, g_{1}, g_{1}^{\prime} \in G_{\alpha 0}^{\prime}$, then $g g^{\prime}=e^{\mathfrak{I}+\mathfrak{l}^{\prime}} g_{1}^{\prime \prime}$ for some $g_{1}^{\prime \prime} \in G_{\alpha 0}^{\prime}$ because $G_{\alpha 0} / G_{\alpha 00}^{\prime}$ is abelian. Therefore

$$
c_{\omega 0}\left(g g^{\prime}\right)=c_{\alpha 0}(g)+c_{\alpha 0}\left(g^{\prime}\right), \quad g, g^{\prime} \in G_{\alpha 00} .
$$

Next, we define $c_{\alpha 0}(g)$ for $g \in G_{(\infty)}$ by (8.45). If $g=e^{\mathrm{l}} g_{1}=e^{\mathrm{I}^{\prime}} g_{1}^{\prime}$, $\mathfrak{l}, \mathfrak{l}^{\prime} \in \mathfrak{g}_{(x)}, g_{1}, g_{1}^{\prime} \in G_{c 0}$, then $e^{-\mathfrak{l}} e^{\mathfrak{l}^{\prime}}=g_{1}\left(g_{1}^{\prime}\right)^{-1} \in G_{a 0}$ and hence $\mathfrak{l}^{\prime}-\mathfrak{l} \in \mathfrak{g}_{\alpha 00}$ 。 Let $\mathfrak{I}^{\prime \prime} \equiv \int_{0}^{1} \operatorname{Ad}\left(e^{-\beta \mathfrak{l}}\right)\left(\mathfrak{l}^{\prime}-\mathfrak{l}\right) \mathrm{d} \beta \in \mathfrak{g}_{x 0}$. Then $e^{-\mathfrak{l}} e^{\mathfrak{l}^{\prime}}=e^{\mathfrak{l}^{\prime \prime}} g_{2}$ for some $g_{2} \in$ $G_{\alpha 0}^{\prime}$. Hence

$$
\begin{aligned}
c_{\infty 0}\left(g_{1}^{*}\right) & =c_{\alpha 0}\left(e^{\mathfrak{I}^{\prime \prime}} g_{2}\right)+c_{\alpha 0}\left(g_{1}^{\prime}\right) \\
& =x_{\alpha}\left(\mathfrak{l}^{\prime \prime}\right)+c_{\alpha 0}\left(g_{1}^{\prime}\right) \\
& =x_{\alpha}\left(\mathfrak{l}^{\prime}-\mathfrak{l}\right)+c_{\alpha 0}\left(g_{1}^{\prime}\right),
\end{aligned}
$$


where the last equality follows from (8.83). Therefore, the definition (8.45) does not depend on the decomposition of $g$.

From (8.83), it follows that

$$
c_{\alpha 0}\left(g g^{\prime} g^{-1}\right)=c_{\alpha 0}\left(g^{\prime}\right)
$$

for $g \in G^{(\alpha)}, \quad g^{\prime} \in G_{\alpha 0}$ as well as for $g \in K_{\alpha}, g^{\prime} \in G_{(\alpha)}$. From the definition and (8.89), we also have

$$
c_{\alpha 0}\left(g g^{\prime}\right)=c_{\alpha 0}(g)+c_{\alpha 0}\left(g^{\prime}\right), \quad g \in G_{(\alpha)}, g^{\prime} \in G_{\alpha 0} .
$$

Suppose $g \in H_{x}, g^{\prime}=e^{\mathfrak{l}} g_{1}, \mathfrak{l} \in \mathfrak{g}_{\alpha}, g_{1} \in G_{x 0}$. Then $g g^{\prime} g^{-1}\left(g^{\prime}\right)^{-1}=g_{2} g_{3}$,

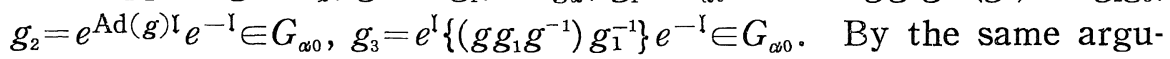
ment as in (8.90), we have $c_{\alpha 0}\left(g_{2}\right)=x_{\alpha}(\operatorname{Ad}(g) \mathfrak{l}-\mathfrak{l})$. From (8.83) and (8.89), we have $c_{\alpha 0}\left(g_{3}\right)=c_{\alpha 0}\left(g g_{1} g^{-1} g_{1}^{-1}\right)=c_{\alpha 0}\left(g g_{1} g^{-1}\right)+c_{\alpha 0}\left(g_{1}^{-1}\right)=c_{\alpha 0}\left(g_{1}\right)-$ $c_{\alpha 0}\left(g_{1}\right)=0$. Therefore

$$
c_{\alpha \curvearrowleft}\left(g g^{\prime} g^{-1}\left(g_{1}^{\prime}\right)^{-1}\right)=x_{\alpha}(\operatorname{Ad}(g) \mathfrak{l}-\mathfrak{l}) .
$$

If $\mathfrak{l}^{\prime} \in \mathfrak{h}_{x}$, then

$$
x_{x}\left(\operatorname{Ad}\left(e^{\mathfrak{l}}\right) \mathfrak{l}-\mathfrak{l}\right)=x_{x}\left(\left[\mathfrak{l}^{\prime}, \mathfrak{l}\right]\right)
$$

due to (8.83). From (8.93) and the commutativity in $G_{(x)} / G_{\alpha 0}$, it follows that

$$
c_{. v 0}\left(g\left(g_{1} g_{2}\right) g^{-1}\left(g_{1} g_{2}\right)^{-1}\right)=c_{x 0}\left(g g_{1} g^{-1} g_{1}^{-1}\right)+c_{x 0}\left(g g_{2} g^{-1} g_{2}^{-1}\right)
$$

if $g_{1}, g_{2} \in G_{(\alpha)}$.

We now define $c_{x 0}(g)$ for $g \in G^{(\alpha)}$ by making decomposition $g=$ $g^{(3)} g^{(2)} g^{(1)}, g^{(1)} \in G_{(x)}, g^{(2)} \in G_{\alpha}^{\prime}, g^{(3)} \in K_{\infty}$ and then by setting

(8. 96) $\quad c_{x 0}(g)=c_{\alpha+1}\left(g^{(3)} g^{(2)}\right)+c_{\alpha 0}\left(g^{(1)}\right)+(1 / 2) c_{\alpha 0}\left(g^{(2)} g^{(1)}\left\{g^{(2)}\right\}^{-1}\left\{g^{(1)}\right\}^{-1}\right)$.

We want to show the independence of this definition on the decomposition of $g$.

Let $g=g_{a}^{(3)} g_{a}^{(2)} g_{a}^{(1)}=g_{b}^{(3)} g_{b}^{(2)} g_{b}^{(1)}$ be two decompositions of $g$. Since $K_{x} \cap H_{\alpha}=H_{\alpha 0}, g_{a}^{(3)}=g_{0}^{(3)} g^{\prime}$ for some $g^{\prime} \in H_{\alpha i 0}$. Let $g^{\prime} g_{a}^{(2)} \equiv g_{c}^{(2)}$. Then

$$
x_{\alpha}\left(\operatorname{Ad}\left(g_{c}^{(2)}\right) \mathfrak{l}\right)=x_{\alpha}\left(\operatorname{Ad}\left(g_{\alpha}^{(2)}\right) \mathfrak{l}\right)
$$

for any $\mathfrak{l} \in \mathfrak{g}_{\imath}$ due to (8.83) and $g^{\prime} \in H_{x 0} \subset K_{x}$. Therefore $c_{\alpha 0}(g)$ defined by two decompositions $g=g_{a}^{(3)} g_{a}^{(2)} g_{a}^{(1)}=g_{b}^{(3)} g_{c}^{(2)} g_{a}^{(1)}$ is the same. Next, we note that $g_{c}^{(2)}=g_{b}^{(2)} g^{\prime \prime}, g_{b}^{(1)}=g^{\prime \prime} g_{a}^{(1)}$ for $g^{\prime \prime}=\left(g_{b}^{(2)}\right)^{-1} g_{c}^{(2)}=$ $g_{b}^{(1)}\left(g_{\alpha}^{(1)}\right)^{-1} \in G_{(\alpha)} \cap G_{\alpha}^{\prime}=G_{\alpha 0}$. From (8.92) and (8.91), we have 


$$
c_{\alpha 00}\left(g_{b}^{(1)}\right)=c_{\alpha 0}\left(g_{a}^{(1)}\right)+c_{\alpha 0}\left(g^{\prime \prime}\right) .
$$

Since $g^{\prime \prime} \in G_{x 0} \subset G_{(x+1) 0}$, we have from the inductive assumption and its consequence (8.49)

$$
c_{(a+1)}\left(g_{b}^{(3)} g_{c}^{(2)}\right)=c_{(\alpha+1)}\left(g_{b}^{(3)} g_{b}^{(2)}\right)+c_{\left(x_{b}+1\right)}\left(g^{\prime \prime}\right) .
$$

We note that $c_{(\alpha+1)}\left(g^{\prime \prime}\right)=c_{(\alpha+1) 0}\left(g^{\prime \prime}\right)=c_{\alpha 0}\left(g^{\prime \prime}\right)$ by our assumption (8.84). From (8.95), we have

$$
\begin{aligned}
& c_{\alpha 0}\left(g_{b}^{(2)} g_{b}^{(1)}\left(g_{b}^{(2)}\right)^{-1}\left(g_{b}^{(1)}\right)^{-1}\right)=c_{\alpha 0}\left(g_{b}^{(2)} g_{a}^{(1)}\left(g_{b}^{(2)}\right)^{-1}\left(g_{a}^{(1)}\right)^{-1}\right) \\
& \quad+c_{\alpha 00}\left(g_{b}^{(2)} g^{\prime \prime}\left(g_{b}^{(2)}\right)^{-1}\left(g^{\prime \prime}\right)^{-1}\right)
\end{aligned}
$$

where the second term vanishes due to the special case $\mathfrak{l}=0$ of (8.93).

Finally we note that $x_{\alpha 0}\left(\operatorname{Ad}\left(g_{b}^{(2)}\right) \mathfrak{l}\right)=x_{a 0}\left(\operatorname{Ad}\left(g^{\prime \prime \prime}\right)^{-1} \operatorname{Ad}\left(g_{c}^{(2)}\right) \mathfrak{l}\right)=$ $x_{\infty 0}\left(\operatorname{Ad}\left(g_{c}^{(2)}\right) \mathfrak{l}\right)$ for any $\mathfrak{l} \in G_{(\alpha)}$ due to $g^{\prime \prime \prime} \equiv g_{c}^{(2)} g^{\prime \prime}\left(g_{c}^{(2)}\right)^{-1} \in G_{\alpha 0} \subset K_{\infty}$ and (8.83). Hence

(8. 101) $\quad c_{\alpha 0}\left(g_{c}^{(2)} g_{a}^{(1)}\left(g_{c}^{(2)}\right)^{-1}\left(g_{a}^{(1)}\right)^{-1}\right)=c_{\alpha}\left(g_{b}^{(2)} g_{a}^{(1)}\left(g_{b}^{(2)}\right)^{-1}\left(g_{a}^{(1)}\right)^{-1}\right)$.

Combining (8.98), (8.99), (8.100) and (8.101), we have the independence of $c_{a 0}(g)$ for two decompositions $g=g_{b}^{(3)} g_{c}^{(2)} g_{a}^{(1)}=g_{b}^{(3)} g_{b}^{(2)} g_{b}^{(1)}$.

Q.E.D.

To sum up our result, we can obtain all possible pairs $\phi$ and $c$ from the subgroup structure (1), $x_{\infty}$ and $\mu_{\alpha}$. $x_{\alpha}$ determines $c$ and the imaginary part of the inner product of $\phi$ while $\mu_{\infty}$ determines the real part of the inner product of $\phi$. In order that our construction works, it is necessary and sufficient for $x_{\alpha}$ and $\mu_{\alpha}$ to satisfy the invariance (8.83), the mutual compatibility (8.84), the positivity of $\mu_{a}$ and the inequality (8.82).

\section{§9. Examples}

(1) Abelian group.

In this case we have both $B^{1}\left(G, \bar{D}^{+}\right)$and $Z^{1}\left(G, L_{2}(R, \mu)\right)$ where $\mathfrak{R}=\mathfrak{g}+i \mathrm{~g}$. Since $\operatorname{Ad}(G)=1, \mu$ can be arbitrary positive definite real inner product. Imaginary part for $\left(\phi_{1}^{a}\left(g_{1}\right), \phi_{1}^{a}\left(g_{2}\right)\right)$ is impossible and $e^{i c}$ is a character. We write a general elements of $G$ by $f=\left(f_{1} \cdots f_{n}\right)$ and denote the group operation by vector addition. Then the general form of $F$ in Theorem 5.1 is 
(9. 1) $\quad F(f)=-\sum_{j, k=1}^{n} f_{j} \mu_{j k} f_{k}+i \sum_{j=1}^{n} c_{j} f_{j}$

$$
+\int\left(\exp i \sum_{j=1}^{n} f_{j} p_{j}-1-i \sum_{j=1}^{n} f_{j} p_{j} g(p)\right) \frac{1+p^{2}}{p^{2}} \mathrm{~d} \mu(p)
$$

where $\mu_{j k}$ is a real positive semidefinite matrix, $c_{j}$ is a real number, $\mu$ is a finite positive measure, $p^{2}=\sum_{j=1}^{n}\left(p_{j}\right)^{2}$ and $g(p)$ is any smooth function with $g(0)=1$ and decreasing rapidly at $p \rightarrow \infty$. A different choice of $g$ is equivalent to a different choice of $c_{j}$. The exact form of $\frac{\left(1+p^{2}\right)}{p^{2}}$ is not important, because any bounded change can be absorbed in $\mu$.

If $X=R^{N}$, we may represent elements in $\mathrm{C}(G, X)$ by $\mathrm{f} \equiv\left(\mathrm{f}_{1}(x)\right.$, $\left.\cdots, \mathrm{f}_{n}(x)\right)$. Then the continuous tensor product part of a uniformly continuous $\sigma$ factorizable separable functional $\mathrm{E}$ can be written as

$$
\mathrm{E}(f)=\exp \int F_{x}(\mathrm{f}(x)) \mathrm{d} \nu(x)
$$

where $F_{x}$ is of the form (9.1) and $\mu_{j k}, c_{j}$ and $\mu$ depends on $x$, where $\nu$ is a continuous positive measure. (To obtain this form of an integral, we may use the standard reduction theory of $\&$ relative to $\{\mathrm{P}(Y)\}$.)

The first term and the last integral in (9.1) defines two mutually orthogonal exponent space $\mathfrak{L}_{a}$ and $\mathfrak{R}_{b}$. The representation space is the $e^{\mathfrak{R}_{a}} \otimes e^{\mathfrak{R}_{b}}$ and the representing unitary operator is of the form $U_{a}(\mathrm{f}) \otimes U_{b}(\mathrm{f}) . \quad \mathbb{Q}_{a}$ is the direct sum of $n$ copies of $R^{N}$ with the inner product

$$
\left(\mathrm{f}^{1}, \mathrm{f}^{2}\right)=2 \sum_{j k} \int \mathrm{f}_{j}^{1}(x) \mu_{j k}(x) \mathrm{f}_{k}^{2}(x) \mathrm{d} \nu(x) .
$$

In terms of creation and annihilation operators, $\mathrm{U}_{a}(f)$ can be written as

$$
\begin{gathered}
\mathrm{U}_{a}(\mathrm{f})=\chi_{a}(\mathrm{f}) \exp i\left\{\left(\mathrm{a}_{a}^{*}, \mathrm{f}\right)+\left(\mathrm{f}, \mathrm{a}_{a}\right)\right\}, \\
\left(\mathrm{a}_{a}^{*}, \mathrm{f}\right)=\sum_{j} \int \mathrm{a}_{a j}(x)^{*} \mathrm{f}_{j}(x) \mathrm{d} x .
\end{gathered}
$$

The second term of (9.1) gives the $c$ number coefficients

$$
\chi_{a}(f)=\exp i \int \sum_{j=1}^{n} c_{j}(x) f_{j}(x) \mathrm{d} x .
$$


$\mathfrak{Q}_{b}$ is the $\mathscr{Q}_{2}$ space on the space of points $\left(x_{1} \cdots x_{N}, p_{1} \cdots p_{n}\right), x \in R^{N}$, $p \in R^{n}$ with respect to $\mathrm{d}_{\mu_{x}}(p) \mathrm{d} \nu(x) . Q(f)$ is the multiplication of the function $\exp i \sum_{j=1}^{n} \mathrm{f}_{j}(x) p_{j}$ and $\Omega \in \bar{D}^{+}$is represented by a function $\left(1+p^{2}\right)^{1 / 2} /\left(p^{2}\right)^{1 / 2} \equiv \Omega(p)$. In terms of creation and annihilation operator, $\mathrm{U}_{b}(\mathrm{f})$ can be written as

$$
\begin{aligned}
\mathrm{U}_{b}(\mathrm{f})= & \exp i \int \mathrm{d} \mu_{x}(p) \mathrm{d} \nu(x)\left\{\left(\mathrm{a}_{b}(x, p)^{*}+\Omega(p)\right)\right. \\
& \left.\times\left(\mathrm{a}_{b}(x, b)+\Omega(p)\right)-\Omega(p)^{2} g(p)\right\} \sum_{j=1}^{n} \mathrm{f}_{j}(x) p_{j} .
\end{aligned}
$$

The special case of (9.1) for $n=1$ is given in [2] and is known as Lévy-Kinchin formula. The equation (9.7) gives a representation of the Poisson process part essentially in terms of the number operator on the Hilbert space of the Gaussian process. (However, the creation and annihilation operators are displaced by $\Omega(p)$, and the number is weighted by $p_{j}$.)

This situation prevails for a general case, as can be seen from Lemma 4.2. The representing operator $U\left(e^{\mathfrak{l} \chi_{Y}}\right)$ is of the form (9.7) for $\phi$ in $B^{1}\left(G, \bar{D}^{+}(G)\right)$ and of the form (9.4) for $\phi_{a}^{a}$ and $g \in H_{a}$.

(2) Canonical commutation relation ( $C C R$ ).

The group $G$ is the Heisenberg group given by the multiplication rule

$$
\left(a_{1}, b_{1}, c_{1}\right)\left(a_{2}, b_{2}, c_{2}\right)=\left(a_{1}+a_{2}, b_{1}+b_{2}, c_{1}+c_{2}+a_{2} b_{1}\right)
$$

where $a, b, c$ are real numbers. The commutator group $G_{c}$ of $G$ coincide with the center of $G$ and consists of $(0,0, c)$. We may take it as our $G_{2}$. For $C C R$, there is an additional requirement that (0, $0, c)$ is represented by a $c$-number $e^{i c}$. Thus $B^{1}\left(G, \bar{D}^{+}\right)$part is actually $B^{1}\left(G / G_{c}, \bar{D}^{+}\right)$and exactly the same as the commutative case. Also by the condition that $(0,0, c)$ is represented by $e^{i c}, \phi_{j}(g)=0$ for $j=2$. For $j=3, G=G_{3}$ and hence $Q_{3}(g)=1$. Since $c_{2}$ is already fixed for $G_{2}$, the imaginary part of $\left(\phi_{3}\left(g_{1}\right), \phi_{3}\left(g_{2}\right)\right)$ part is fixed to be $\frac{1}{2}\left(a_{1} b_{2}-a_{2} b_{1}\right)$. The real part is arbitrary except for the positive definiteness condition. As a net result, we have

$$
\begin{gathered}
F(a, b, c)=-\mu_{11} a^{2}-\mu_{12} a b-\mu_{22} b^{2}-\frac{i}{2} a b+i c+i(\alpha a+\beta b) \\
\quad+\int\left\{\exp i\left(a p_{1}+b p_{2}\right)-1-i\left(a p_{1}+b p_{2}\right) g(p)\right\} \frac{1+p^{2}}{p^{2}} \mathrm{~d} \mu(p),
\end{gathered}
$$




$$
\mu_{11}>0, \quad 4 \mu_{11} \mu_{22} \geq\left(\mu_{12}\right)^{2}+(1 / 4) .
$$

This form was obtained by more elementary consideration in [1]. For $E(f)$, we have an expression of the form (9.2).

The exponent space can be split into a direct sum of 3 spaces $\mathbb{R}^{(j)}, j=1,2,3$. The representation space is $e^{\Omega^{(1)}} \otimes e^{\Omega^{(2)}} \otimes e^{\mathfrak{R}^{(3)}}$ and the representing operator is $\mathrm{U}_{1}(\mathrm{f}) \otimes \mathrm{U}_{2}(\mathrm{f}) \otimes \mathrm{U}_{3}(\mathrm{f})$. $\quad \mathcal{R}^{(1)}$ is the space of $\{f(x)$, $g(x)$, both $f$ and $g$ being real, with an inner product

$$
\begin{gathered}
\left(\left\{f_{1}(x), g_{1}(x)\right\},\left\{f_{2}(x), g_{2}(x)\right\}\right)=2 \int\left\{f_{1}(x) f_{2}(x) \mu_{11}(x)\right. \\
+g_{1}(x) g_{2}(x) \mu_{22}^{\prime}(x)+\frac{1}{2}\left(f_{1}(x) g_{2}(x)+f_{2}(x) g_{1}(x) \mu_{12}(x)\right. \\
\left.+\frac{i}{4}\left(f_{1}(x) g_{2}(x)-f_{2}(x) g_{1}(x)\right)\right\} \mathrm{d} \nu(x), \\
\mu_{22}^{\prime}(x)=\left\{4 \mu_{11}(x)\right\}^{-1}\left|\mu_{12}(x)+\frac{i}{2}\right| .
\end{gathered}
$$

This is related to the standard form of the Fock representation of $C C R$ by a Bogoliubov transformation. $\mathcal{Z}^{(2)}$ is the space of $\{g(x)\}, g$ being complex, with the inner product

$$
\left(g_{1}(x), g_{2}(x)\right)=2 \int g_{1}(x)^{*} g_{2}(x)\left(\mu_{22}(x)-\mu_{22}^{\prime}(x)\right) \mathrm{d} \nu(x) .
$$

The $\phi(g)$ in this space corresponds to a real wave function. The splitting between $\mathfrak{Q}^{(1)}$ and $\mathfrak{Q}^{(2)}$ is rather arbitrary, for example we may leave a part of $\mu_{11}$ in $\mathfrak{Q}^{(2)}$ and modify $\mu_{22}^{\prime}$ accordingly. In any case the creation and annihilation operator of the original $C C R$ is represented by a linear combination of two kinds of Fock creation and annihilation operators. If we choose the splitting appropriate, it can be arranged as a hole and a particle, namely the creation operator of $C C R$ is represented as a sum of Fock creation operator of one space (particle) and Fock annihilation operator of another space (hole). A typical example is in [4]. The $\mathcal{Q}^{(3)}$ part is exactly the same as the commutative case where the relevant group is that of $(a, b, 0)$ and is two dimensional.

(3) The current algebra over rotation group.

We consider $G=S U(2)$ or $0(3)$. Then the group is compact and hence $Z^{1}(G, L)=B^{1}(G, L)$. In this case we have a representation 
$\mathrm{U}(g)=e^{i H(\mathfrak{l})}, g=e^{\mathfrak{l}}$ in a Fock space $e^{\mathfrak{Q}}$ where $\mathfrak{L}=\int^{\oplus} \mathbb{Q}_{x} \mathrm{~d} \nu(x)^{1 / 2}, Q(g)=$ $\int^{\oplus} Q_{x}(g), \Omega=\int^{\oplus} \Omega_{x}$ and

(9. 13) $\quad H(\mathfrak{l})=-i \int\left(\mathrm{a}^{*}(x)+\Omega(x), \mathrm{d} Q_{x}(\mathfrak{l})(\mathrm{a}(x)+\Omega(x))\right) \mathrm{d} \nu(x)$.

\section{Acknowledgement}

The author would like to thank Professor O. Takenouchi for critical reading of the manuscript and helpful comments.

\section{References}

[1] Araki, H., Princeton Thesis, 1960.

[2] Gelfand, I. M. and N. Ya. Vilenkin, Generalized Functions, Vol. 4, Chapter 3, Section 4, Academic Press, 1964.

[3] Araki, H. and J. Woods, Publ. RIMS, Ser. A 2 (1966), 157-242.

[4] Araki, H. and J. Woods, J. Math. Phys. 4 (1963), 637-662.

[5] Riesz, F. and B. Sz.-Nagy, Leçons d'Analyse Fonctionnelle, Académie des Sciences de Hongrie, 1952. 